\title{
The Herschel/PACS view of the Cep OB2 region: Global protoplanetary disk evolution and clumpy star formation $\star, \star \star$
}

\author{
Aurora Sicilia-Aguilar ${ }^{1,2}$, Veronica Roccatagliata ${ }^{3}$, Konstantin Getman ${ }^{4}$, Pablo Rivière-Marichalar ${ }^{5,2}$, \\ Tilman Birnstiel ${ }^{6}$, Bruno Merín ${ }^{7}$, Min Fang ${ }^{2}$, Thomas Henning ${ }^{8}$, Carlos Eiroa $^{2}$, and Thayne Currie ${ }^{9}$
}

1 SUPA, School of Physics and Astronomy, University of St Andrews, North Haugh, St Andrews KY16 9SS, UK e-mail: asa5@st-andrews.ac.uk

2 Departamento de Física Teórica, Facultad de Ciencias, Universidad Autónoma de Madrid, 28049 Cantoblanco, Madrid, Spain

3 Universitäts-Sternwarte München, Ludwig-Maximilians-Universität,Scheinerstr. 1, 81679 München, Germany

4 Department of Astronomy \& Astrophysics, 525 Davey Laboratory, Pennsylvania State University, University Park, PA 16802, USA

5 Kapteyn Astronomical Institute, PO Box 800, 9700 AV Groningen, The Netherlands

${ }^{6}$ Harvard-Smithsonian Center for Astrophysics, 60 Garden Street, Cambridge, MA 02138, USA

7 Herschel Science Centre, ESAC-ESA. PO Box 78, 28691 Villanueva de la Cañada, Madrid, Spain

8 Max-Planck-Institut für Astronomie, Königstuhl 17, 69117 Heidelberg, Germany

9 Department of Astronomy \& Astrophysics, University of Toronto, Canada

Received 24 July 2014 / Accepted 8 October 2014

\section{ABSTRACT}

\begin{abstract}
Context. The Cep OB2 region, with its two intermediate-aged clusters $\operatorname{Tr} 37$ and NGC 7160, is a paradigm of sequential star formation and an ideal site for studies of protoplanetary disk evolution.

Aims. We use Herschel data to study the protoplanetary disks and the star formation history of the region.

Methods. Herschel/PACS observations at 70 and $160 \mu \mathrm{m}$ probe the disk properties (mass, dust sizes, structure) and the evolutionary state of a large number of young stars. Far-IR data also trace the remnant cloud material and small-scale cloud structure.

Results. We detect 95 protoplanetary disks at $70 \mu \mathrm{m}, 41$ at $160 \mu \mathrm{m}$, and obtain upper limits for more than 130 objects. The detection fraction at $70 \mu \mathrm{m}$ depends on the spectral type ( $88 \%$ for K4 or earlier stars, $17 \%$ for M3 or later stars) and on the disk type ( $\sim 50 \%$ for full and pre-transitional disks, $\sim 35 \%$ for transitional disks, no low-excess/depleted disks detected). Non-accreting disks are not detected, suggesting significantly lower masses. Accreting transition and pre-transition disks have systematically higher $70 \mu \mathrm{m}$ excesses than full disks, suggestive of more massive, flared and/or thicker disks. Herschel data also reveal several mini-clusters in Tr 37, which are small, compact structures containing a few young stars surrounded by nebulosity.

Conclusions. Far-IR data are an excellent probe of the evolution of disks that are too faint for sub-millimetre observations. We find a strong link between far-IR emission and accretion, and between the inner and outer disk structure. Herschel confirms the dichotomy between accreting and non-accreting transition disks. Accretion is a powerful measure of global disk evolution: substantial mass depletion and global evolution need to occur to shut down accretion in a protoplanetary disk, even if the disk has inner holes. Disks likely follow different evolutionary paths: low disk masses do not imply opening inner holes, and having inner holes does not require low disk masses. The mini-clusters reveal multi-episodic star formation in $\operatorname{Tr} 37$. The long survival of mini-clusters suggest that they formed from the fragmentation of the same core. Their various morphologies favour different formation/triggering mechanisms acting within the same cluster. The beads-on-a-string structure in one mini-cluster is consistent with gravitational fragmentation or gravitational focusing, acting on very small scales (solar-mass stars in $\sim 0.5 \mathrm{pc}$ filaments). Multi-episodic star formation could also produce evolutionary variations between disks in the same region. Finally, Herschel also unveils what could be the first heavy mass loss episode of the O6.5 star HD 206267 in $\operatorname{Tr} 37$.
\end{abstract}

Key words. stars: pre-main sequence - protoplanetary disks - stars: formation - open clusters and associations: individual: CepOB2 circumstellar matter - stars: individual: HD 206267

\section{Introduction}

The Cep OB2 region is a classical example of sequential or triggered star formation (Patel et al. 1998). Located at $870 \mathrm{pc}$ distance (Contreras et al. 2002), it contains the young clusters $\operatorname{Tr} 37$

^ Based on observations obtained with the Herschel Space Telescope within open time proposal "Disk dispersal in Cep OB2", OT1_asicilia_1. Herschel is an ESA space observatory with science instruments provided by European-led PI consortia and with important participation from NASA.

$\star \star$ Appendices are available in electronic form at

http://www . aanda.org and NGC 7160 (Platais et al. 1998). With mean ages 4 Myr for Tr 37 and 12 Myr for NGC 7160 (Sicilia-Aguilar et al. 2005), the two regions are an ideal place for the study of protoplanetary disk evolution at critical ages for disk dispersal (Sicilia-Aguilar et al. 2006a; Hernández et al. 2007). Spitzer IRAC and MIPS surveys have been used to study the disk population among the cluster members (Sicilia-Aguilar et al. 2006a; Mercer et al. 2009 [M09]; Morales-Calderón et al. 2009 [MC09]; Getman et al. 2012 [G12]; Sicilia-Aguilar et al. 2013b [SA13]), estimating a disk fraction $\sim 48 \pm 5 \%$ for solar-type stars (spectral types late G/early M) in $\operatorname{Tr} 37$, while only two disks are found among the more than 60 NGC 7160 low-mass members. Spitzer photometry 
also revealed significant disk evolution, with the mean disk excesses being lower than in Taurus (Sicilia-Aguilar et al. 2006a). Spitzer/IRS and IRAM millimetre data allowed us to impose stronger constraints on the evolution of the disks, estimating the total disk mass in small dust, and revealing grain growth and inside-out evolution (Sicilia-Aguilar et al. 2007, 2011).

Nevertheless, observations in the near- and mid-IR have limited power to reveal the structure of the disk as a whole. For evolved disks in the distant Cep OB2 region, single-dish submillimetre data are often unfeasible because of low fluxes, cloud contamination, and beam dilution. Constraining the evolutionary status of a disk is a complex problem, given the high degree of degeneracy between nearly all important parameters involved in disk evolution (grain size, disk flaring, settling, presence of inner holes and gaps; e.g. D'Alessio et al. 2004, 2005; Dullemond \& Dominik 2004; Whitney et al. 2013). Far-IR data is thus a bridge between the inner and outer disk properties, and a key to study global disk evolution, including global small dust depletion, settling, and changes in the grain size distribution. Herschel/PACS data allow us to obtain details about the global mass and structure of faint and very evolved disks, which typically have (sub-)millimetre fluxes that are too low to be detected.

If Cep OB2 is a paradigm of sequential star formation (Patel et al. 1995), $\operatorname{Tr} 37$ is an example of multi-episodic star formation. IC $1396 \mathrm{~A}$ is clearly younger than the main $\operatorname{Tr} 37$ cluster (Sicilia-Aguilar et al. 2005, 2006a, 2014, from now on Paper I). In addition, previous Spitzer and optical studies suggested the presence of smaller structures containing $<10$ spatially-related, stars (Barentsen et al. 2001 [B11]; G12; SA13), which are probably younger than the main cluster population attending to their disk features and high accretion rates. These grouplets or miniclusters are a reduced version of the sub-clusters found in massive star-forming regions (MYStiX survey; Getman et al. 2014; Kuhn et al. 2014), although the latter are denser, presumably more numerous, and larger in size than the mini-clusters. Miniclusters extend the idea of clumpy star formation in molecular clouds down to less massive, less dense regions. Triggering (Getman et al. 2014) or sequential formation of stars in a nonuniform cloud may offer an answer to such structures. Herschel data allows us to track cooler (compared to Spitzer) and less dense (compared to millimetre observations) cloud structures, revealing the details of the star formation history of the region.

In Paper I, we discussed the young IC 1396 A globule in $\operatorname{Tr} 37$, and associated embedded protostars. Here we present the results of our Herschel/PACS survey of disks and cloud structure in the Tr 37 and NGC 7160 clusters. Observations and data reduction are discussed in Sect. 2. In Sect. 3, we analyse the disk properties and evolutionary status as seen by Herschel and the cluster structure. In Sect. 4, we discuss the evolutionary signs observed in the disks in the context of the dispersal of the gaseous and dusty disk and its potential outcomes, and the possibility of multi-episodic star formation in $\operatorname{Tr} 37$. Finally, our results are summarised in Sect. 5.

\section{Observations and data reduction}

\subsection{Herschel/PACS observations and map-making}

The Cep OB2 clusters, Tr 37 (centered at 21:38:09, +57:26:48 J2000) and NGC 7160 (centered at 21:53:40, 62:36:10 J2000), were observed with the ESA Herschel Space Observatory (Pilbratt et al. 2010) using the Photodetector Array Camera and Spectrometer (PACS; Poglitsch et al. 2010), as part of the open time (OT) program "Disk dispersal in Cep OB2" (PI A. Sicilia-Aguilar). We obtained a total of $23 \mathrm{~h}$ observing time in parallel mode at 70 and $160 \mu \mathrm{m}$. Table 1 lists the astronomical observation requests (AORs), pointings, sensitivity, and map areas. Observations comprise six scan maps and 19 mini-maps. Large scan maps were designed to cover most of the cluster members and the extended emission, while mini-maps were selected to trace fainter sources with deeper integration. There is some overlap at the edges between the maps that increase the sensitivity in localized areas. For the large maps, we took two AORs per pointing, corresponding to scan and cross-scan (at 45 and 135 degrees with respect to the array). For mini-maps, we observed two AORs per pointing with scan and cross-scan at 70 and 110 degrees with respect to the array. Observations took place between November 2012 and January 2013.

The data were reduced using $\mathrm{HIPE}^{1}$ environment, version 9.0 (Ott et al. 2010) and the Unimap ${ }^{2}$ software (Piazzo et al. 2012). We use the PACS data calibration of 2013 February 14. Unimap is recommended for PACS observations of sources with complex background (Paladini et al. 2013 ${ }^{3}$ ). The strongest extended emission in the region is associated with the IC $1396 \mathrm{~A}$ globule (Paper I). The sources presented in this work are point-like (disks) or small $\left(<15^{\prime \prime}\right)$ extended structures. For intermediate-brightness sources, the differences in the photometry resulting from HIPE and Unimap maps are negligible, but very faint sources are washed out if iterative masking techniques are applied, or artificially enhanced if independent masking is provided within HIPE. The map construction starts from Level 0 products, flags bad and saturated pixels, converts ADUs to volts, and computes the coordinates with aberration correction. Unimap processes the standard Level 1 products using a generalised least squares approach (GLS) to estimate the noise spectrum. It also fixes cosmic rays, glitches, and drifts. The initial GLS map is subsequently corrected from the distortion introduced by GLS, finally resulting in post-processed, weighted GLS (WGLS) maps. The final maps are projected with pixel sizes $2^{\prime \prime}$ for the $70 \mu \mathrm{m}$ image, and $3^{\prime \prime}$ for the $160 \mu \mathrm{m}$ image using the UniHIPE software, also available from Unimap. Overlapping AORs of the large scan maps were combined together. Combining large maps and mini-maps does not significantly improve the signal-to-noise ratio $(\mathrm{S} / \mathrm{N})$ of sources far from the centre, so we treated both kinds of maps separately. Figure 1 shows some parts of Tr 37 observed with Herschel/PACS.

\subsection{Point source photometry}

We performed point source aperture photometry for all the known young stars with disks in Cep OB2 located within our Herschel/PACS fields. A list of spectroscopically confirmed cluster members was drawn from Sicilia-Aguilar et al. 2004, 2005, 2006b, and SA13. We also analysed other candidate members based on Spitzer IR excesses, $\mathrm{H} \alpha$ emission, and X-ray emission (M09; MC09; B11; G12). The IRAF ${ }^{4}$ package apphot was

1 HIPE is a joint development by the Herschel Science Ground Segment Consortium, consisting of ESA, the NASA Herschel Science Center, and the HIFI, PACS and SPIRE consortia.

2 See http://w3. uniroma1.it/unimap/for further details.

3 http://herschel.esac.esa.int/twiki/pub/Public/

PacsCalibrationWeb/pacs_mapmaking_report_ex_sum_v3.pdf 4 IRAF is distributed by the National Optical Astronomy Observatories, which are operated by the Association of Universities for Research in Astronomy, Inc., under cooperative agreement with the National Science Foundation. 

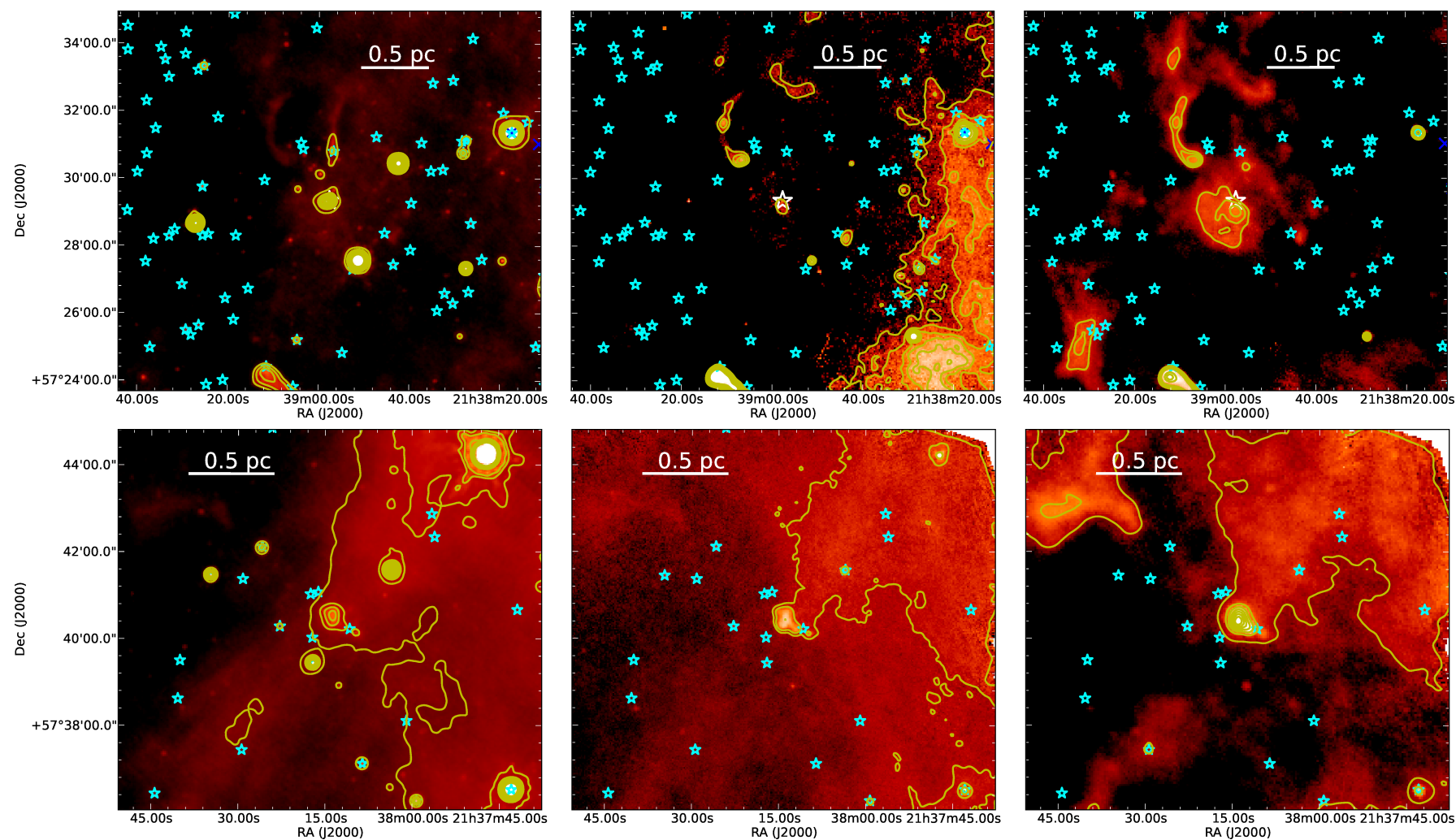

Fig. 1. Images at 24, 70, and $160 \mu \mathrm{m}$ (from left to right) of the centre (up) and the northern part (down) of Tr 37 . The O6.5 star HD 206267 is marked with a large white star in the upper diagrams. Other cluster members (with and without disks) are marked with small cyan stars. The extended object at 21:38:14, +57:40:27 is likely an unrelated planetary nebula (based on cluster age considerations).

Table 1. Herschel/PACS AORs and fields.

\begin{tabular}{|c|c|c|c|c|c|c|c|c|}
\hline Field & AOR numbers & $\begin{array}{c}\text { RA } \\
(\mathrm{J} 2000)\end{array}$ & $\begin{array}{c}\text { Dec } \\
(\mathrm{J} 2000)\end{array}$ & $\begin{array}{l}\text { Duration } \\
\text { (s) }\end{array}$ & Date & Type & Size & $\begin{array}{c}1 \sigma_{70 \mu \mathrm{m}} / 1 \sigma_{160 \mu \mathrm{m}} \\
(\mathrm{mJy})\end{array}$ \\
\hline $01-580$ & $1342261383 / 4$ & $21: 53: 37.07$ & $+62: 28: 47.0$ & $2 \times 895$ & 2013-01-18 & $\mathrm{M}$ & $6.5^{\prime} \times 2.5^{\prime}$ & $1.1 / 3.0$ \\
\hline $01-1152$ & $1342261385 / 6$ & $21: 53: 19.83$ & $+62: 34: 00.5$ & $2 \times 445$ & 2013-01-18 & M & $6.5^{\prime} \times 2.5^{\prime}$ & $1.8 / 4.1$ \\
\hline DG-481 & $1342261387 / 8$ & $21: 52: 21.13$ & $+62: 45: 03.5$ & $2 \times 445$ & 2013-01-18 & M & $6.5^{\prime} \times 2.5^{\prime}$ & $1.8 / 4.1$ \\
\hline North & $1342256224 / 5$ & $21: 38: 20.15$ & $+57: 41: 01.6$ & $2 \times 2244$ & 2012-11-29 & $\mathrm{S}$ & $7.0^{\prime} \times 7.0^{\prime}$ & $2.5 / 5.6$ \\
\hline West & $1342256961 / 2$ & $21: 35: 18.90$ & $+57: 32: 15.0$ & $2 \times 4563$ & 2012-12-11 & $S$ & $10.5^{\prime} \times 10.5^{\prime}$ & $2.3 / 5.1$ \\
\hline $72-875$ & $1342256963 / 4$ & $21: 35: 49.75$ & $+57: 24: 04.1$ & $2 \times 895$ & $2012-12-11$ & M & $6.5^{\prime} \times 2.5^{\prime}$ & $1.1 / 3.0$ \\
\hline $54-1781$ & $1342258024 / 5$ & $21: 38: 16.13$ & $+57: 19: 35.8$ & $2 \times 445$ & 2013-01-01 & M & $6.5^{\prime} \times 2.5^{\prime}$ & $1.8 / 4.1$ \\
\hline $23-798$ & $1342259517 / 8$ & $21: 41: 28.65$ & $+57: 36: 43.3$ & $2 \times 445$ & 2013-01-11 & M & $6.5^{\prime} \times 2.5^{\prime}$ & $1.8 / 4.1$ \\
\hline IC 1396A & $1342259791 / 2$ & $21: 37: 07.24$ & $+57: 29: 41.9$ & $2 \times 3101$ & 2013-01-16 & $\mathrm{S}$ & $8.0^{\prime} \times 8.0^{\prime}$ & $2.4 / 5.4$ \\
\hline Cluster & $1342259793 / 4$ & 21:38:18.77 & $+57: 31: 36.8$ & $2 \times 10082$ & 2013-01-16 & $\mathrm{S}$ & $12.5^{\prime} \times 12.5^{\prime}$ & $1.6 / 3.6$ \\
\hline East & $1342259795 / 6$ & $21: 39: 58.97$ & $+57: 32: 36.1$ & $2 \times 8156$ & 2013-01-17 & S & $13.5^{\prime} \times 13.5^{\prime}$ & $2.0 / 4.3$ \\
\hline 93-720 & $1342261393 / 4$ & $21: 40: 10.00$ & $+58: 00: 03.7$ & $2 \times 445$ & 2013-01-18 & M & $6.5^{\prime} \times 2.5^{\prime}$ & $1.8 / 4.1$ \\
\hline 21393104 & $1342261395 / 6$ & $21: 39: 31.05$ & $+57: 47: 14.0$ & $2 \times 445$ & 2013-01-18 & M & $6.5^{\prime} \times 2.5^{\prime}$ & $1.8 / 4.1$ \\
\hline $91-506$ & $1342261397 / 8$ & $21: 38: 58.07$ & $+57: 43: 34.4$ & $2 \times 445$ & 2013-01-18 & M & $6.5^{\prime} \times 2.5^{\prime}$ & $1.8 / 4.1$ \\
\hline $92-393$ & $1342261399 / 400$ & $21: 39: 44.08$ & $+57: 42: 16.0$ & $2 \times 895$ & 2013-01-18 & M & $6.5^{\prime} \times 2.5^{\prime}$ & $1.1 / 3.0$ \\
\hline KUN-196 & $1342261401 / 2$ & $21: 40: 15.09$ & $+57: 40: 51.3$ & $2 \times 445$ & 2013-01-18 & M & $6.5^{\prime} \times 2.5^{\prime}$ & $1.8 / 4.1$ \\
\hline $24-1796$ & $1342261403 / 4$ & $21: 40: 11.83$ & $+57: 40: 12.2$ & $2 \times 445$ & 2013-01-18 & M & $6.5^{\prime} \times 2.5^{\prime}$ & $1.8 / 4.1$ \\
\hline $13-1250$ & $1342261431 / 2$ & $21: 39: 12.14$ & $+57: 36: 16.5$ & $2 \times 445$ & 2013-01-18 & M & $6.5^{\prime} \times 2.5^{\prime}$ & $1.8 / 4.1$ \\
\hline $21-998$ & $1342261433 / 4$ & $21: 39: 34.80$ & $+57: 23: 27.8$ & $2 \times 445$ & 2013-01-18 & M & $6.5^{\prime} \times 2.5^{\prime}$ & $1.8 / 4.1$ \\
\hline $21-33$ & $1342261435 / 6$ & $21: 39: 35.62$ & $+57: 18: 22.1$ & $2 \times 445$ & 2013-01-18 & M & $6.5^{\prime} \times 2.5^{\prime}$ & $1.8 / 4.1$ \\
\hline 21362507 & $1342261853 / 4$ & $21: 36: 25.08$ & $+57: 27: 50.3$ & $2 \times 445$ & 2013-01-23 & M & $6.5^{\prime} \times 2.5^{\prime}$ & $1.8 / 4.1$ \\
\hline $11-1209$ & $1342261855 / 6$ & $21: 36: 58.51$ & $+57: 23: 25.8$ & $2 \times 445$ & 2013-01-23 & M & $6.5^{\prime} \times 2.5^{\prime}$ & $1.8 / 4.1$ \\
\hline $12-1091$ & $1342261857 / 8$ & 21:37:57.62 & $+57: 22: 47.7$ & $2 \times 445$ & 2013-01-23 & M & $6.5^{\prime} \times 2.5^{\prime}$ & $1.8 / 4.1$ \\
\hline 54-1547 & $1342261859 / 60$ & 21:38:44.46 & $+57: 18: 09.1$ & $2 \times 445$ & $2013-01-23$ & M & $6.5^{\prime} \times 2.5^{\prime}$ & $1.8 / 4.1$ \\
\hline 21391145 & $1342261861 / 2$ & 21:39:07.50 & $+57: 23: 52.6$ & $2 \times 541$ & 2013-01-23 & $\mathrm{S}$ & $4.5^{\prime} \times 3.0^{\prime}$ & $3.0 / 6.9$ \\
\hline
\end{tabular}

Notes. List of the observed fields and information about the pointings. The first three pointings belong to the cluster NGC 7160, while the rest belong to $\operatorname{Tr} 37$. The size of the fields correspond approximately to the places with high coverage. The $1 \sigma$ value corresponds to the average sensitivity estimated by Hspot at the centre of the map. The local sensitivity decreases near extended structures or at the edges of the maps, and increases in the combined map in regions covered by more than one map. The map type is marked as scan-map (S) or mini-map (M). 
used to automatically calculate the aperture photometry on the source position (considering 2MASS coordinates), to subtract the sky, and to derive the corresponding errors. Since the background is highly variable throughout most of the fields, we used small apertures ( $5^{\prime \prime}$ for $70 \mu \mathrm{m}, 8^{\prime \prime}$ for $\left.160 \mu \mathrm{m}\right)$, together with the aperture corrections (1.764 for $70 \mu \mathrm{m}, 1.919$ for $160 \mu \mathrm{m})$ recommended by $\mathrm{Herschel}^{5}$. Sky annuli were placed between 12-18" for the $70 \mu \mathrm{m}$ maps, and $18-27^{\prime \prime}$ for the $160 \mu \mathrm{m}$ maps. The aperture corrections are derived considering larger sky annuli, but we adopted a compromise between sky location and the strongly variable background found in these regions (see also SiciliaAguilar et al. 2013a). The errors are calculated taking into account the average sky rms and the correlated noise due to undersampling, estimated as (3.2/pixsize $)^{0.68} / 0.95$, where pixsize is the pixel size in the projected maps (A. Mora, priv. comm.). We also include a general error of $10 \%$ to account for flux calibration, aperture, and mapping effects on the final flux. This error is consistent with the typical variations observed in the photometry of intermediate-brightness sources derived from maps constructed with different tools (Unimap vs. HIPE standard scripts). Colour corrections for objects with relatively flat $v F_{v}$ distributions are very small compared to the flux errors (see PACS calibration manual ${ }^{6}$; Howard et al. 2013), so the photometry is presented uncorrected "as is".

Automated algorithms for finding point sources often fail in far-IR images with extended structures. Therefore, in addition to checking source positioning, centering, and $\mathrm{S} / \mathrm{N}$ (detections must be $\geq 3 \sigma$ over the background), we did a careful visual inspection to control that the sources were real and point-like. We do not expect the disks to have extended emission (the PACS point spread function, PSF, at $70 \mu \mathrm{m}$ is $5.6^{\prime \prime}$, equivalent to $\sim 5000 \mathrm{AU}$ at $870 \mathrm{pc}$ ), so extended sources are regarded as "cloud contamination" and thus non-detections. Special attention was paid to sources showing coordinate mismatches $>2$ " that could be a sign of misidentification with red background sources. We only consider as real Herschel detections those belonging to objects with IR excesses (from Spitzer and/or WISE data) in their spectral energy distributions (SED). Sources with apparent PACS counterparts but no IR excess are probably spurious because the Herschel detection limits are several orders of magnitude over the photospheric emission of late-type stars or associated cold/debris disks. For completeness, we explored objects with marginal excess at 8 and/or $24 \mu \mathrm{m}$ only, but none of them is detected with Herschel. Some of the $\mathrm{H} \alpha$ candidates in B11 $(\sim 15 \%)$ do not show any disk emission at Spitzer wavelengths, and are thus not considered.

Source detectability varies greatly across the maps, depending on the presence of cloud/background emission. For nondetected sources in clean areas, we can estimate significant upper limits. For sources associated with bright regions such as IC $1396 \mathrm{~A}$, the upper limits are of the order of Jy and thus do not offer any further constraint to the SEDs. Sources at the edge of the maps also have uncertain upper limits and are not considered. For cases with low, smooth background, we estimated a $3 \sigma$ upper limit to the flux considering the sky rms at the position of the source. For a few objects, the automated photometry classified them as detections, but visual inspection of the maps revealed that although there is some emission consistent with the source, the local background structures (e.g. nebular patches,

\footnotetext{
5 http://herschel.esac.esa.int/twiki/pub/Public/ PacsCalibrationWeb/pacs_bolo_fluxcal_report_v1.pdf

6 http://herschel.esac.esa.int/twiki/pub/Public/ PacsCalibrationWeb/cc_report_v1.pdf
}

nearby bright objects) could affect the detectability. We consider these as "marginal detections", since the source appears to be detected and its flux could be of the same order than the "marginal" detection, but there is a high risk of contamination.

The photometry for all objects and their SEDs are presented in Appendix A. From our spectroscopically identified sources, (483 in Tr 37 and 145 in NGC 7160, many of which are out of the Herschel fields), we detect 77 at $70 \mu \mathrm{m}$ (only one in NGC 7160), 36 of them also at $160 \mu \mathrm{m}$. All detections are listed in Table A.1. The only object detected in NGC 7160 is the only surviving accreting star in this cluster. From the 270 targets from M09, MC09, B11, and G12 (many of which are outside the Herschel fields), 18 are detected at $70 \mu \mathrm{m}$ and five of them are also detected at $160 \mu \mathrm{m}$. All these objects are listed in Table A.2. Two further objects, $21373786+5728467$ and $21373885+5732494$, have tentative far-IR detections, but since the rest of data does not show disk-like excesses, we classify them as most likely background-contaminated. We also obtain significant upper limits for two objects with disks in NGC 7160 and 117 more in Tr 37, among spectroscopically-identified members (Table A.3). A further 25 objects without spectroscopic confirmation have significant upper limits (Table A.4). Their properties (including membership confirmation, spectral type, and presence of accretion) are less well established than for spectroscopically confirmed members, but the strong selection criteria offered by $\mathrm{X}$-ray, IR excess, and $\mathrm{H} \alpha$ photometry ensure a very reduced potential contamination by non-members.

The maps also reveal several tens of point sources that have not been classified as young stellar objects (YSO) by previous surveys. Because of the thorough search for YSO in the centre of the clusters (optical, X-ray, $\mathrm{H} \alpha$, and Spitzer/WISE searches from various teams), most of the new point sources probably correspond to extragalactic objects, so we are leaving them out of the current work.

\subsection{Ancillary data}

There is a large collection of ancillary data available for most sources, including optical photometry and spectroscopy (Contreras et al. 2002; Sicilia-Aguilar et al. 2004, 2005; SA13; B11), 2MASS near-IR photometry (Cutri et al. 2003), Spitzer IRAC/MIPS fluxes (Sicilia-Aguilar et al. 2006a; SA13; M09; MC09; G12). Objects with optical spectroscopy and photometry also have spectral type, extinction estimates, and strong constraints on the presence of accretion. For a subset of objects with accurate spectral types and extinction, individual, instantaneous accretion rates are also available (Sicilia-Aguilar et al. 2010). Finally, for a few objects we also have Spitzer/IRS spectra and $1.3 \mathrm{~mm}$ continuum emission measurements and/or upper limits (Sicilia-Aguilar et al. 2007, 2011). Many objects have been detected with WISE, but we give priority to Spitzer data, since WISE photometry is strongly affected by source blending and cloud emission in distant and relatively crowded fields. All the Spitzer photometry for the sources presented here was derived self-consistently following the procedures in (SA13).

All the available data ${ }^{7}$ is used to trace the SEDs of the sources (Sect. 3.1). The data are non-simultaneous. Optical variability is common in young stars (Herbst et al. 2000; Briceño et al. 2001; Sicilia-Aguilar et al. 2005), but most of the CepOB2 objects do not seem to be strongly variable in the IR. In a few cases such as 21-998, 21394850+572049544, and $21395813+5728335$, the SEDs betray potential IR variability.

7 Ancillary data available upon request. 

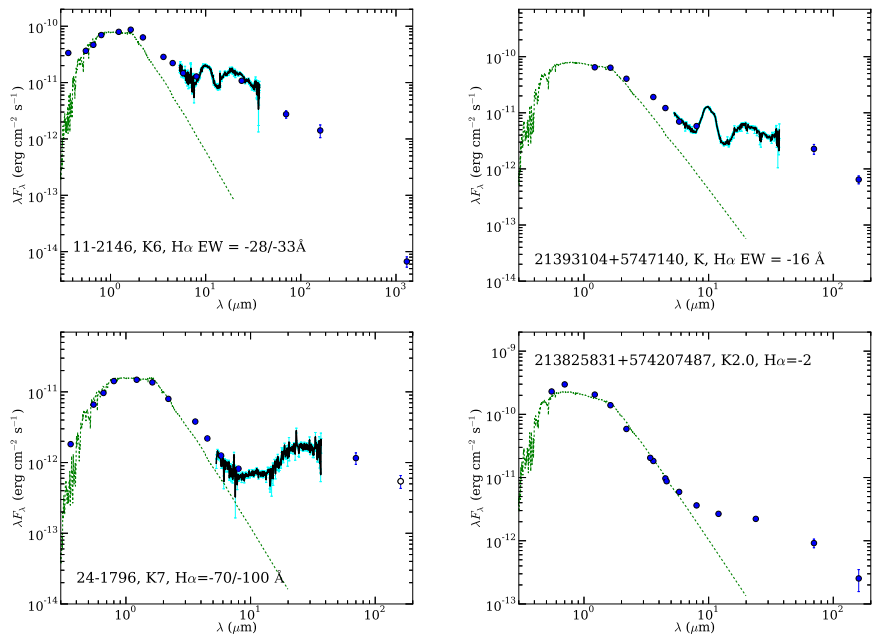
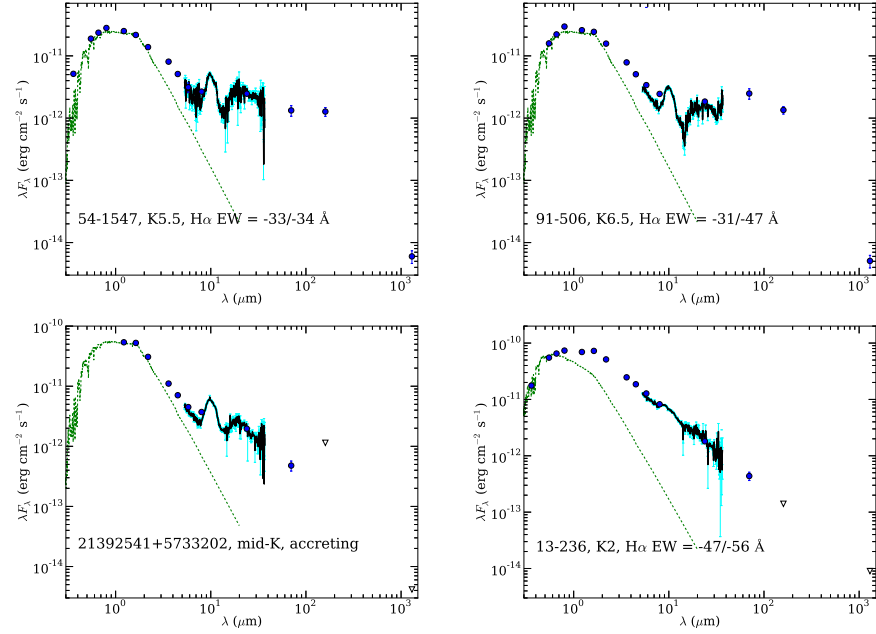

Fig. 2. Examples of SEDs of objects detected with Herschel, including optical, Spitzer (IRAC/MIPS photometry and IRS spectra), WISE, and IRAM $1.3 \mathrm{~mm}$ data. Errorbars are often smaller than the symbols (full circles). Upper limits are marked as inverted open triangles, marginal detections are open circles. In the upper row, 11-2146 and 21393104+5747140 are full disks, 54-1547 and 91-506 are pre-transitional disks. In the lower row, 24-1796 and 213825831+574207487 are transitional disks, and 21392541+5733202 and 13-236 are disks with signs of strong dust processing/low mass in small grains. Spectral types and $\mathrm{H} \alpha$ equivalent width in emission are also listed. A MARCS model photosphere (Gustafsson et al. 2008) is shown as a dotted line for comparison.

Table 2. SED classification.

\begin{tabular}{lcl}
\hline \hline Disk class & Criteria & Interpretation \\
\hline Transition disk (TD) & {$[3.6]-[4.5]<0.2 \mathrm{mag}$} & $\begin{array}{l}\text { Inner hole, radial variations of dust properties } \\
\text { and/or settling, inside-out evolution. }\end{array}$ \\
Kink/Pre-transition disk (PTD) & $\begin{array}{c}\text { [3.6]-[4.5] 0.2-0.4 mag, } \\
\text { strong silicate, } \\
\text { and sharp slope change }\end{array}$ & $\begin{array}{l}\text { Holes/gaps with small grains, } \\
\text { inside-out evolution, } \\
\text { dust filtering by planet/companion. }\end{array}$ \\
Low excess/Depleted disk (D) & $\begin{array}{c}\text { [3.6]-[4.5] 0.2-0.4 mag, } \\
\text { and similar slope up to } 24 \mu \mathrm{m}\end{array}$ & $\begin{array}{l}\text { Small dust depletion, strong settling, } \\
\text { global disk evolution. }\end{array}$ \\
& None of the above & Consistent with homogeneous, continuous, flared CTTS disk. \\
\hline
\end{tabular}

Notes. The SED classification used in the paper, together with the different physical interpretations of the SED morphology in each disk class.

For very variable sources, the SED classification may be uncertain.

\section{Analysis}

\subsection{SEDs and disk classification}

The disks were classified following the scheme in SA13 according to their Spitzer fluxes and after a careful inspection of their SEDs. Table 2 lists the criteria for SED classification and the possible interpretations in terms of disk structure and evolutionary status. For low-mass stars (spectral types $\mathrm{G}-\mathrm{M}$ ), our classification distinguishes between full disks (with excesses at all wavelengths), transitional disks with inside-out evolution (TD; with nearly photospheric colours, [3.6]-[4.5] < $0.2 \mathrm{mag}$, and large excesses at longer wavelengths), "kink" or pre-transitional disks (PTD; with moderate-to-low near-IR excesses, [3.6]-[4.5] 0.2-0.4 mag, and strong silicate features and/or a sharp change in slope between $8-12$ and $24 \mu \mathrm{m}$ ), and depleted/low-excess disks (with near-IR excesses in the range of PTD but a steep slope at all wavelengths, resulting in low $24 \mu \mathrm{m}$ excesses; Currie et al. 2009). The classification is not unique, as some of the depleted/low-excess disks also satisfy the criteria for TD. For the high-mass stars, the classification was done by visual inspection of the SEDs and comparison to stellar templates (MARCS models, Gustafsson et al. 2008). Class I sources in IC 1396 A were presented in Paper I. For a few objects, where part of the data is missing or inconsistent (usually, due to nearby bright objects, cloud emission, or IR variability), we adopt an "uncertain" SED type and exclude them from the discussion.

The Herschel far-IR fluxes are in very good agreement with the Spitzer photometry and IRS spectra. Figure 2 shows examples of disks with different SEDs types. We find consistently lower Herschel fluxes for objects with low mid-IR excesses, showing that $24 \mu \mathrm{m}$ data offer a good prediction of the global disk structure. This is reflected in the detection rates of objects: while more than half (46 out of 89) of the disks classified as "full" are detected (11 out of 17 in case of PTD), only $1 / 3$ of the TD (19 out of 54) and none of the settled/depleted/low-excess disks (out of 19) is detected. The detection rate is strongly dependent on the spectral type: the only star for which we reach the photospheric levels at $70 \mu \mathrm{m}$ is the O6.5 star HD 206267. We detect some debris disk candidates around A and B stars, but none around late-type stars. For low-mass stars with Spitzerconfirmed disks and spectral types K4 or earlier, the detection rate is close to $90 \%$ (15 out of 17 ), but it drops to $\sim 50 \%$ for 
Table 3. Common parameters in the RADMC disk models.

\begin{tabular}{ll}
\hline \hline Parameter & Value \\
\hline Stellar mass & $M_{*}=1 M_{\odot}$ \\
Effective temperature & $T_{\text {eff }}=4275 \mathrm{~K}$ \\
Stellar radius & $R_{*}=1.7 R_{\odot}$ \\
Disk outer radius & $R_{\text {out }}=200 \mathrm{AU}$ \\
Gas-to-dust ratio & $R_{\text {gas } / \text { dust }}=100$ \\
\hline
\end{tabular}

Notes. The parameters are representative for a young K6 star. The outer disk radius is largely unconstrained with the available data. Deviations from the standard gas-to-dust ratio could also happen in evolved disks.

late-K stars (26 out of 49 ) and to $<30 \%$ for M-type stars (23 out of 55 for early-M stars, four out of 24 for stars with spectral types M3 or later). Therefore, predictions for stars with spectral types $\mathrm{K}$ and earlier are representative for the whole class, while conclusions on the lowest-mass population are subject to higher uncertainty and concern only the most massive and flared disks.

The $160 \mu \mathrm{m}$ fluxes are sometimes higher than expected from the SED shape and the $70 \mu \mathrm{m}$ data, especially for objects with non-negligible background. There is extended $160 \mu \mathrm{m}$ emission in some parts of the cluster (see Fig. 1), and some grouplets of stars are also associated with small nebular structures (see Sect. 3.3). Therefore, even in well-detected sources, extended nebulosity may contaminate the beam at long wavelengths. Extended nebular material could also affect some of the IRAM/1.3 mm detections.

\subsection{The Cep OB2 disks as seen by Herschel: disk structure and its Far-IR imprint}

To examine the far-IR properties of disks in a more quantitative way, we define the spectral indices $\alpha$ as the SED slope between two wavelengths $\lambda_{1}$ and $\lambda_{2}$ (Lada et al. 1987):

$\alpha\left(\lambda_{1}-\lambda_{2}\right)=\frac{\log \left(\lambda_{1} F_{\lambda_{1}}\right)-\log \left(\lambda_{2} F_{\lambda_{2}}\right)}{\log \left(\lambda_{1}\right)-\log \left(\lambda_{2}\right)}$

where $F_{\lambda_{x}}$ is the flux at wavelength $\lambda_{x}$. The Spitzer SED spectral indices (or equivalent colour indices) were used to classify the disks according to the mentioned types (Sicilia-Aguilar et al. 2006a, 2011; SA13). Now the Herschel data allow us to explore independently the global disk properties of objects with inner disks in different evolutionary states.

To understand the effect of the disk structure on the far-IR fluxes, we created a set of template models with fixed stellar properties and various disk parameters (see Table 3). For a stellar model, we chose a star with $T_{\text {eff }}=4275 \mathrm{~K}, M_{*}=1 M_{\odot}$, and $R_{*}=1.7 R_{\odot}$, which corresponds approximately to a solar-type star at an age $\sim 3-4$ Myr (Siess et al. 2000), similar to the typical late-K stars in Cep OB2. We then constructed various simple models using the Monte-Carlo radiative-transfer code RADMC (Dullemond \& Dominik 2004), and varying the disk mass (assuming a gas-to-dust ratio 100), disk thickness, flaring law, grain sizes, and power-law exponent of the grain size distribution. The inclination is always assumed to be 45 degrees. We also modelled "kink"/PTD and TD by introducing radial variations of the inner disk rim (adding inner holes), grain properties, and disk structure. The outer disk radius was kept constant and equal to $200 \mathrm{AU}$ (note that in the absence of millimetre data, there is a strong degeneracy between disk radius and disk mass). We also add a small amount of small dust grains in the inner rim to reproduce the typically strong silicate features. The RADMC models assume that dust grains of all sizes are well-mixed and coupled to the gas, although size-independent dust settling can be simulated by modifying the scale height and assuming that the dust is decoupled from the gas in hydrostatic equilibrium. The details of the individual models are described in Appendix B. The models are not constructed to fit any object in particular nor to cover all possible disk structures, but to give a broad view of the disk structures observed in Cep OB2. Figure 3 shows the global effects on the SED shape as the disk structure and properties are varied in a controlled way.

By examining the model SEDs and spectral indices, we can explore the observable effects of various structural and physical changes in the disks, and compare this to the Cep OB2 observations. Figure 4 shows how the SED spectral index changes when various disks parameters are varied: disk mass (for full disks with well-mixed gas and dust), disk flaring law (for disks with constant mass, ranging from non-flared to very flared), disk thickness (for disks with constant mass and assuming a standard flaring law with $H / R \propto R^{1 / 7}$ ), presence of distinct inner disks of increasing sizes (as in PTD, for disks with constant mass and well-mixed dust and gas), and presence of inner holes of increasing sizes (as in TD, for disks with constant mass and well-mixed dust and gas). The data on the Cep OB2 stars is also displayed.

Figure 4 demonstrates the high degree of degeneracy between physical properties of disks when only colour-colour diagrams are used. Disk mass and flaring have a similar effect on the $\alpha$ indices that measure the global far-IR excess, while other parameters like disk thickness and presence of inner holes/gaps (what we call "transitionality") dominate the effect on the mixed mid- and far-IR spectral indices. For most of our stars, $H$ band emission is photospheric (Sicilia-Aguilar et al. 2005). Therefore, $\alpha(\mathrm{H}-70 \mu \mathrm{m})$ is sensitive to the total far-IR excess and thus a good indicator of the global disk mass and disk flaring. Inside-out evolution and "transitionality" can be well traced by $\alpha(8-70 \mu \mathrm{m})$, although this index is in part affected by the strength of the silicate feature. The TD in Cep OB2 show a range of silicate features (with some of them having no or very weak silicate emission, and others having strong features), while PTD have stronger silicate features than normal disks (and also stronger features than our models here; Sicilia-Aguilar et al. 2007, 2011). This can make the observed difference between full and TD/PTD in the $\alpha(8-70 \mu \mathrm{m})$ index stronger than predicted by the models. The behaviour of $\alpha(24-70 \mu \mathrm{m})$ is more complex, being affected by settling, disk mass, and to some extent, "transitionality".

The grain sizes and grain size distribution leave detectable imprints in the SED slope between 70 and $160 \mu \mathrm{m}$ (Fig. 3). Small-grain dominated distributions increase the far-IR flux and the $160 \mu \mathrm{m}$ emission, making $\lambda F_{\lambda}$ comparable at $70 \mu \mathrm{m}$ and $160 \mu \mathrm{m}$. Figure 5 explores the $\alpha(70-160)$ index. Although we expect to detect at $160 \mu \mathrm{m}$ only the most pristine, brighter disks, we find that the SEDs tend to fall on the side of significant grain growth/dust processing. For some objects, such as 54-1547 and 21-2113, the SED shape is consistent with a small-grain dominated disk, but since both cases are associated with some nebular background emission, we cannot exclude contamination. The very tight upper limits imposed in a few targets reveal that some of the disks suffer from very strong grain processing and/or are very depleted of small dust grains at this stage of evolution. Therefore, the large majority of disks in $\operatorname{Tr} 37$ are consistent with substantial populations of grains with sizes well over $100 \mu \mathrm{m}$.

\subsection{Cloud structure and temperature}

Several areas in Tr 37 show extended far-IR emission. The 12 Myr-old cluster NGC 7160, in contrast, is very clean and 

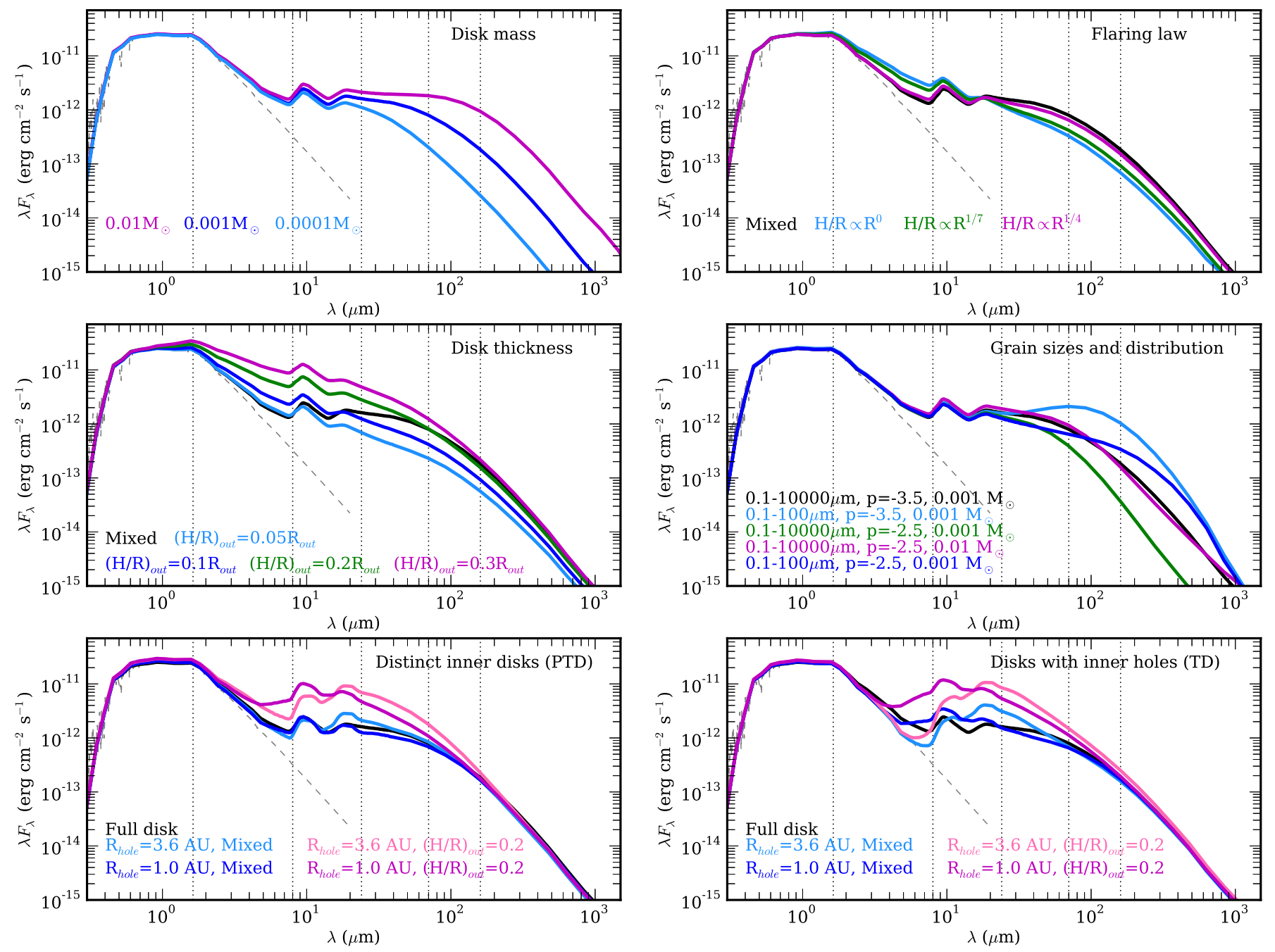

Fig. 3. Comparison of the SEDs of some of the RADMC models in Table B.1. Unless otherwise specified, the models assume well-mixed gas and dust, a disk mass of $0.001 M_{\odot}$ and a standard grain distribution with grain sizes $0.1-10000 \mu \mathrm{m}$ and power-law exponent $p=-3.5$. For examples changing the flaring, thickness, and some of the TD/PTD cases, we show both well-mixed ("Mixed") and decoupled dust/gas distributions (given by various $\mathrm{H} / \mathrm{R}$ relations and outer disk thickness $\left.(\mathrm{H} / \mathrm{R})_{\text {out }}\right)$.

without any signs of extended emission at Spitzer and Herschel wavelengths, suggestive of complete cloud removal at later evolutionary stages. Besides the large IC 1396 A globule (Paper I), we observe substantial patches of nebulosity towards the west and north of $\operatorname{Tr} 37$. They probably correspond to material at the edge of the bubble-shaped HII region surrounding HD 206267 (Patel et al. 1998; B11). The nebular emission is often very inhomogeneous, containing a significant small-scale structure. We also find small $(\sim 0.25 \mathrm{pc})$ and bright nebulous patches, containing several stars with strong mid- and far-IR emission. We call them "mini-clusters" (SA13) and will be described in detail in Sect. 4.2. Figure 6 shows the nebular structures associated with the B3+B5 stars CCDM J2137+5734 (Dommanget et al. 1983; a.k.a. HD 206081; G12), 11-2031, and the clump containing the emission line star 213911452+572425205 (SA13).

The observations at 70 and $160 \mu \mathrm{m}$ can be used to trace the temperature (Paper I) and column density of the cloud structures. Temperatures and densities are only significant in regions dominated by cloud emission, since clean areas are noise-dominated. After resampling both maps at the same resolution $\left(3^{\prime \prime} /\right.$ pixel $)$ and cancelling out fluxes at or below the local noise level (which changes from map to map), the approximate pixel-by-pixel temperature is calculated from the flux ratio by assimilating the emission to a modified blackbody function:

$\frac{F_{v, 70}}{F_{v, 160}}=\frac{B_{v, 70}(T)\left(1-\mathrm{e}^{-\tau_{v, 70}}\right)}{B_{v, 160}(T)\left(1-\mathrm{e}^{-\tau_{v, 160}}\right)}$

Following Roccatagliata et al. (2013), we assume that the emission is optically thin at Herschel/PACS wavelengths, and consider that the optical depth $\tau$ has a power-law dependency with the frequency $(\beta=1.9)$ and is proportional to the mass absorption coefficient $k_{v}$ and the column density $\Sigma$, arriving at:

$F_{v}=\Omega B_{v}(T) \tau_{v}=\Omega B_{v}(T) k_{v} \Sigma$.

Here, $\Omega$ is the solid angle subtended by the emitting region $\left(3^{\prime \prime} \times 3^{\prime \prime}\right.$ pixel). The hydrogen column density $\left(N_{\mathrm{H}}\right)$ is estimated considering the gas-to-dust ratio $\left(R_{\text {gas } / \text { dust }}=100\right)$, the mass of the hydrogen atom $\left(m_{\mathrm{H}}\right)$, and the mean molecular weight $(\mu=2.8)$ :

$N_{\mathrm{H}}=\frac{2 \Sigma R_{\mathrm{gas}} / \mathrm{dust}}{m_{\mathrm{H}} \mu}$.

We can thus calculate the temperature and column density structure of the cloud by fitting the fluxes at 70 and $160 \mu \mathrm{m}$ on a 

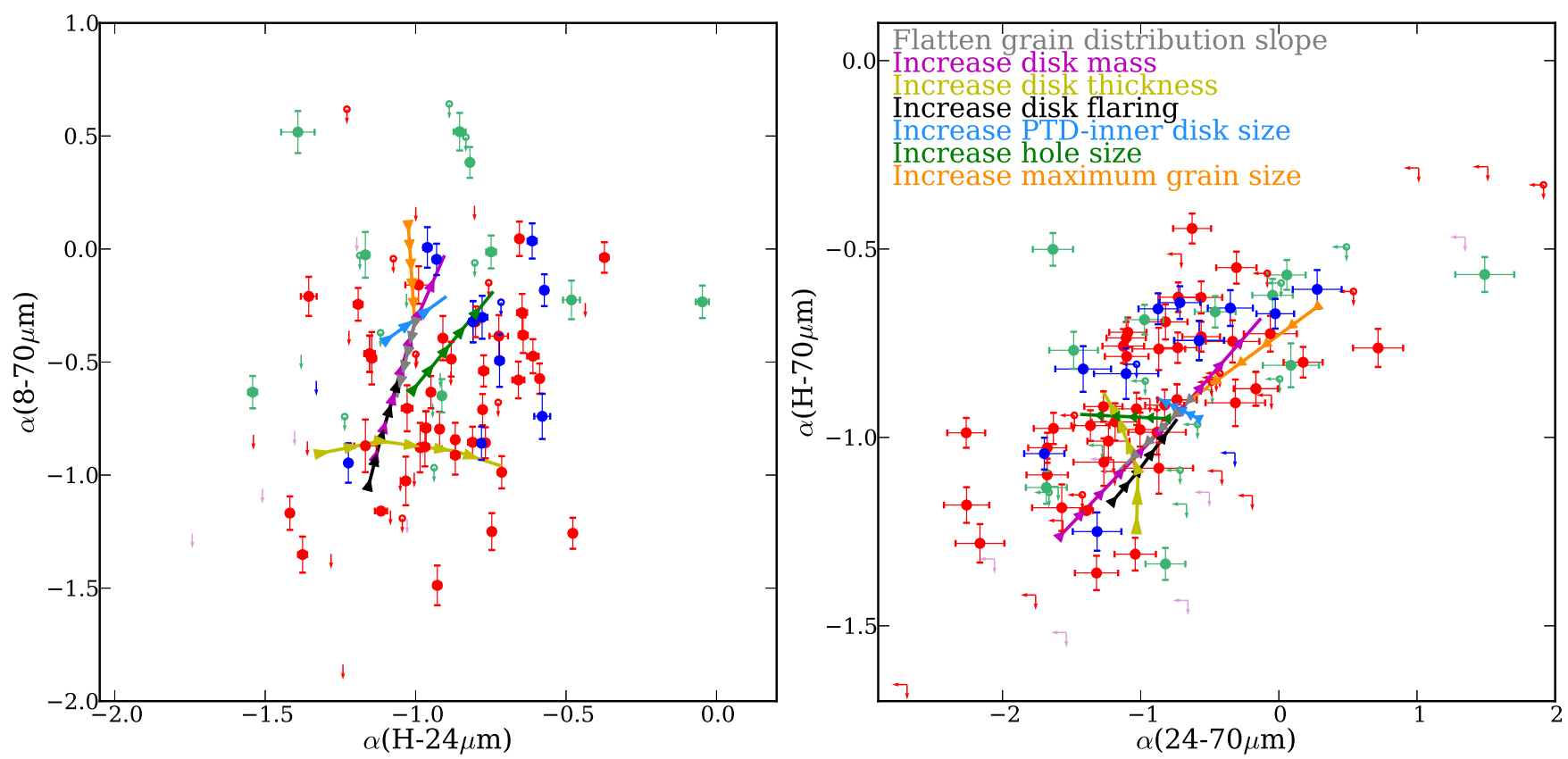

Fig. 4. Mid- and far-IR SED spectral indices for stars with different disk types, classified according to their Spitzer colours. Note the degeneracy that arises between different disk structures when only simple diagrams are considered. Full disks are marked in red, PTD are marked in blue, TD are marked in green, and low excess/depleted disks are marked in pink. Circles represent detections and left/downwards pointing arrows are upper limits. Marginal detections are marked as upper limits with an additional open circle. We only include upper limits for stars with spectral types K7 or earlier, given that the data are very incomplete for M-type stars. The SED spectral indices for the models in Fig. 3 are shown as continuous lines, with arrows marking the direction of the change when various disk properties are modified (see legend).

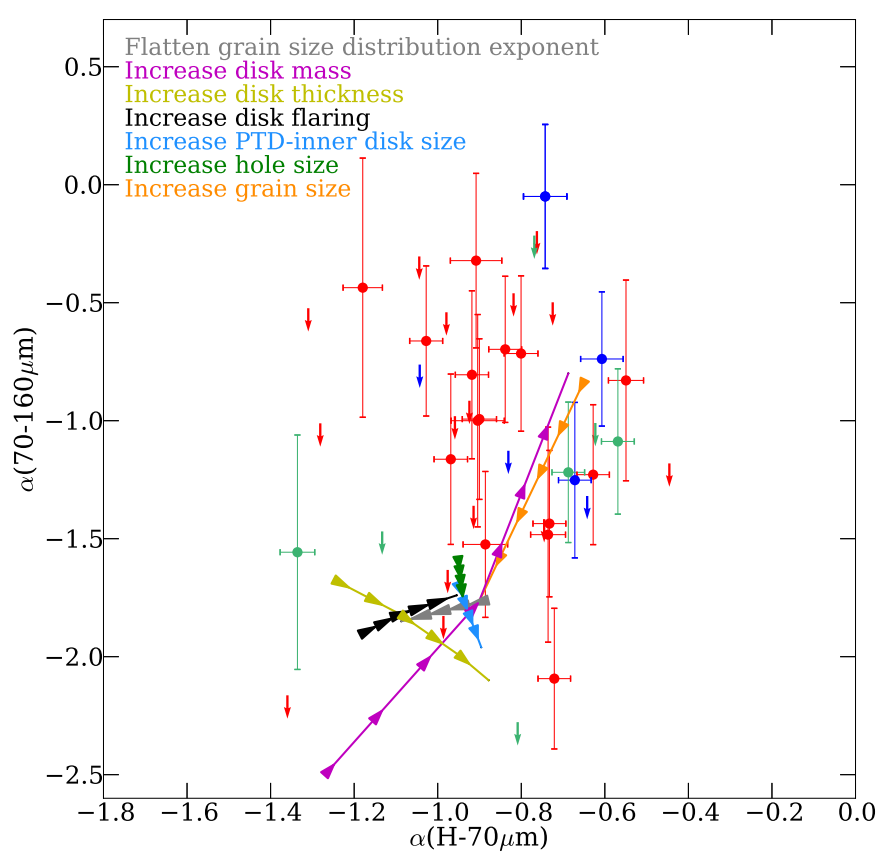

Fig. 5. Observed SED spectral indices $\alpha$ (including $160 \mu \mathrm{m}$ ) and predicted trends from the models in Fig. 3. Detections are marked as dots with errorbars (red for full disks, blue for PTD, green for TD), and upper limits are shown as arrows. We only consider disks detected at $70 \mu \mathrm{m}$ and with no evidence of nebular/extended emission at $160 \mu \mathrm{m}$. The thick lines show the variation of the spectral indices when the disk properties are changed.

pixel-by-pixel base. Since we use two datapoints to fit two parameters, we cannot estimate the uncertainties, but the variations of temperature and column density between neighbouring pixels provide a good idea of the uncertainty levels. This method tends to produce slightly higher temperatures than more complex SED-fitting techniques (Preibisch et al. 2013; Roccatagliata et al. 2013), since the short-wavelength emission is dominated by the hottest parts of the cloud on the line-of-sight. It is also affected by the way in which the dust opacity is treated (Juvela et al. 2013). The cloud is very clumpy and inhomogeneous, so the PACS emission may come from different layers of material at various locations and with different properties. For compact and distinct structures such as mini-clusters, the overdensities observed are probably a more reliable measure of the real column density and temperature of the structure, so they can thus provide information on the heating mechanisms in the region.

Figure 7 shows the temperature and column density structure of the three mini-clusters, together with the extended nebular regions around the centre, west, and north of $\operatorname{Tr} 37$. The temperature is dominated by the massive stars. The 06.5 star HD 206267 is the main source of heating in the cluster, a similar situation to what has been found in more massive star-forming regions (Roccatagliata et al. 2013). Nevertheless, we also observe heating at smaller scales due to the local stellar population. The mini-cluster associated with the binary B star CCDM $\mathrm{J} 2137+5734$ is clearly hotter than the other grouplets, and despite being bright at $24 \mu \mathrm{m}$, it has no significant nebular emission at 70 or $160 \mu \mathrm{m}$. The mini-cluster associated with 11-2031 is, in contrast, better detected at Herschel wavelengths, while the denser clump near the star $213911452+572425205$ has a remarkable cold centre. The northern part of $\operatorname{Tr} 37$ also contains a bright, extended object with an apparent ring-shaped structure and a bipolar outflow (Fig. 8). The structure of the object in the IR and the faint emission in the optical blue bands we observe in our available photometry are consistent with a candidate planetary nebula (Villaver \& Díaz, priv. comm.), probably unrelated to $\operatorname{Tr} 37$ considering the age of the cluster. 

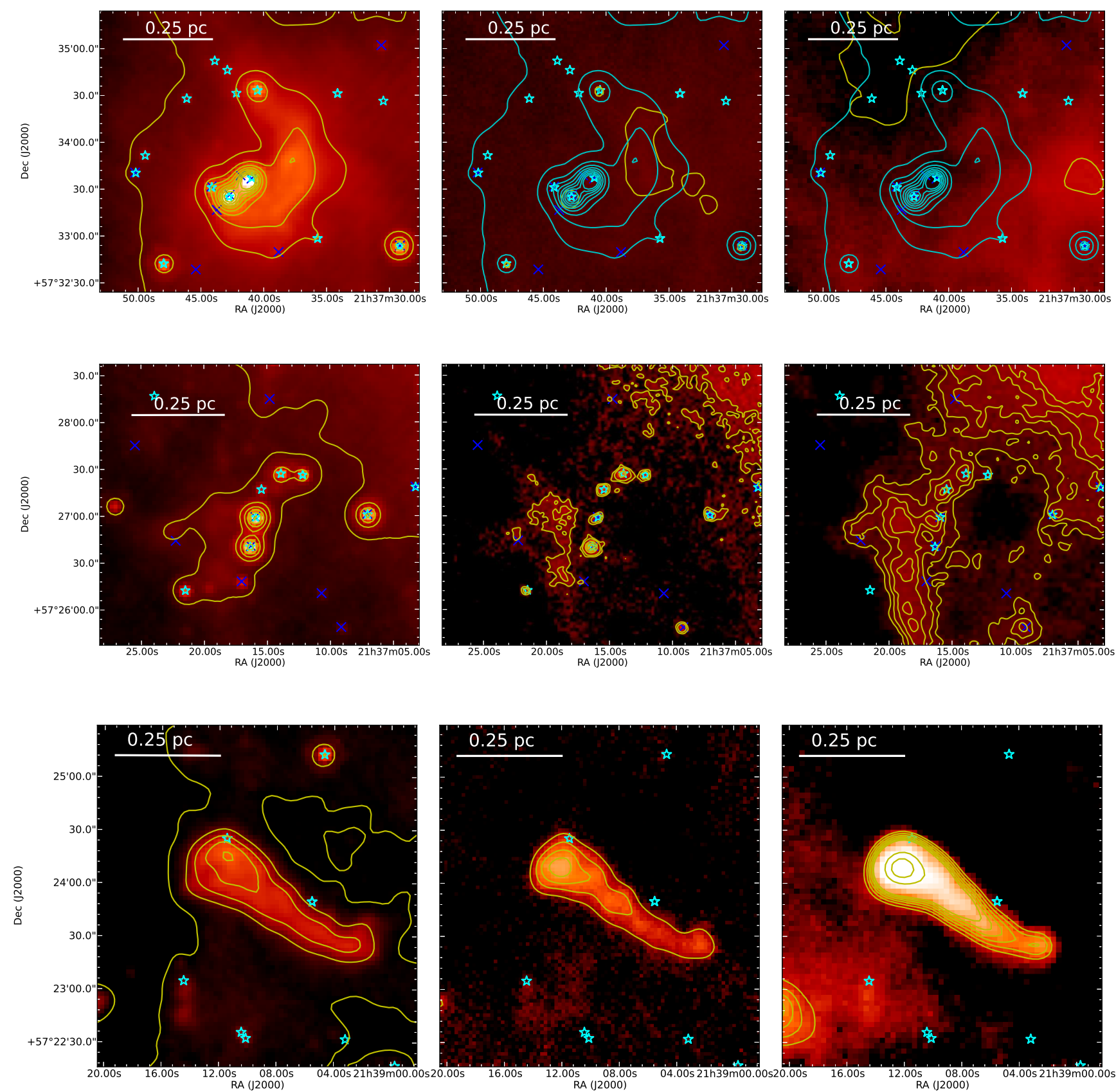

Fig. 6. Images at 24, 70, and $160 \mu \mathrm{m}$ (from left to right) of the mini-clusters associated with CCDM J2137+5734 (top), 11-2031 (middle), and $213911452+572425205$ (bottom). Cluster members (with and without disks) are marked as small cyan stars. Yellow contours at variable levels for each wavelength are shown to mark the structure of the mini-cluster. For the cluster associated with CCDM J2137+5734, whose nebular structure is not detected at Herschel wavelengths, we also overplot the $24 \mu \mathrm{m}$ contours in cyan in the PACS maps to mark the location of the warm dust.

Herschel also reveals some nebulosity around the massive star HD 206267 (a multiple trapezium-like system; Peter et al. 2012). The structure is brighter at $160 \mu \mathrm{m}$ and asymmetric with respect to HD 206267. It does not show evidence of the action of the stellar winds or ionization from the massive star, although the stellar emission merges smoothly onto the extended structure at $160 \mu \mathrm{m}$. The star is known to have strong photoevaporating winds that are sweeping the surrounding $\mathrm{H}$ II region and structures (Sharpless 1959; Osterbrock 1989) and nearby protoplanetary disks (Balog et al. 2006). Elias et al. (2008) also observed a scattering envelope and a well-developed wind around the object. Although chance projection cannot be completely ruled out, it is likely that the observed extended structure corresponds to material ejected by HD 206267. The star is relatively young and has not started yet its phase as a blue variable or WR star, but there are some indications that massive stars may shed larger amounts of mass via strong winds at an early stage. Similar bubble or ring-like structures have been observed in more evolved massive stars with Herschel (Groenewegen et al. 2011; Vamvatira-Nakou et al. 2011, 2013; Cox et al. 2012). There is thus the possibility that Herschel is revealing one of the first heavy mass-loss episodes in HD 206267.

\subsection{Remarkable objects in the Сер ОВ2 region}

The $70 \mu \mathrm{m}$ flux predictions from Spitzer SED models (SiciliaAguilar et al. 2011; SA13) are in good agreement with Herschel 

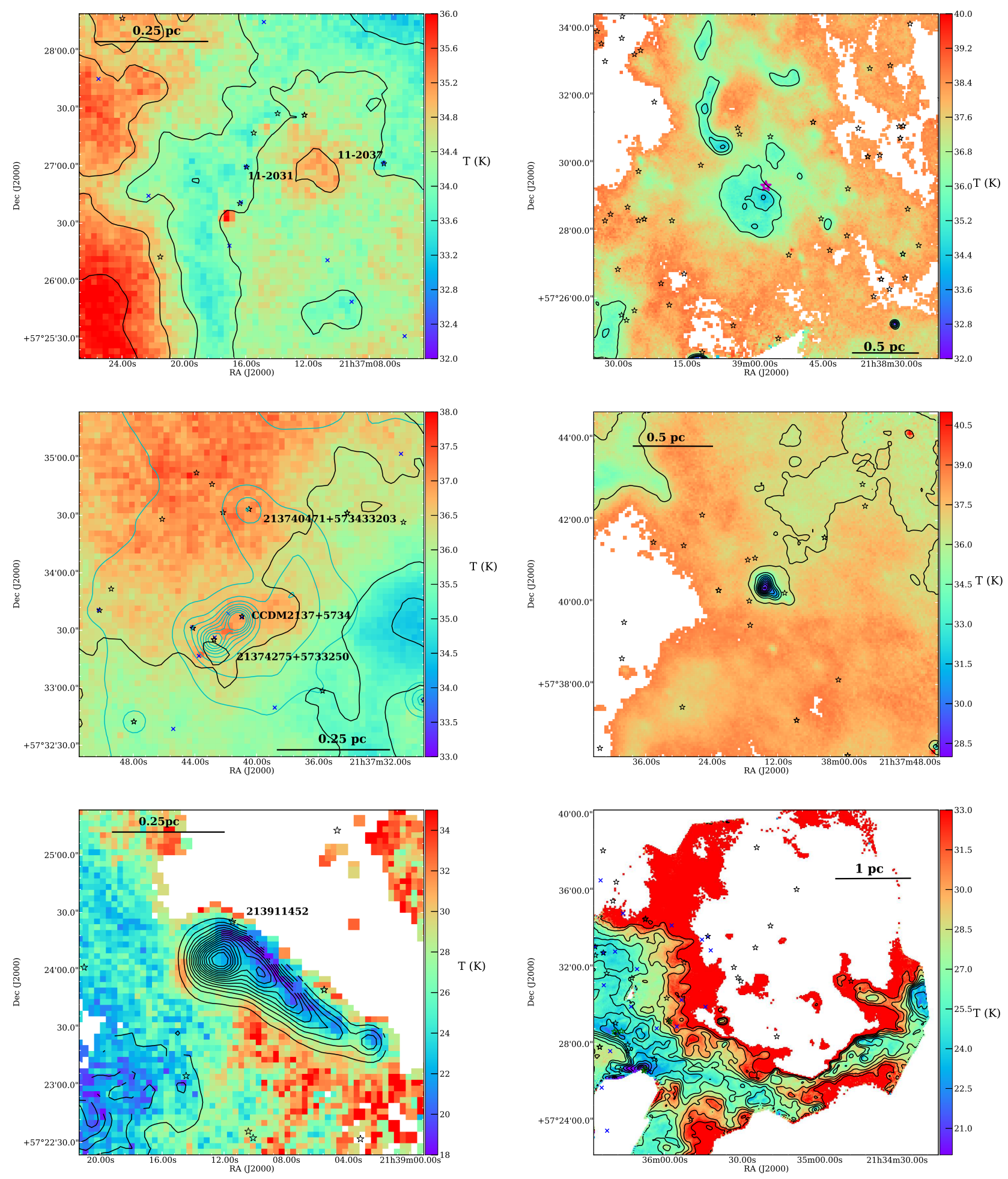

Fig. 7. Approximate temperature maps derived from the Herschel/PACS data for the mini-clusters (left column, top to bottom, mini-clusters associated with: 11-2031, CCDM J2137+5734, and 213911452+572425205) and the centtr, north, and west of Tr 37 (right column, from top to bottom). The $N_{\mathrm{H}}$ column density contours are plotted in black. For the CCDM J2137+5734 mini-cluster, whose structure is not detected by Herschel, we also plot the MIPS $24 \mu \mathrm{m}$ contours (cyan). The temperature and column density scales are different for each region to show the fine structure. A temperature scale is attached to each plot. The $N_{\mathrm{H}}$ contours have been adjusted to each region, and correspond to linear spacing between $2 \mathrm{e}+20-3 \mathrm{e}+20 \mathrm{~cm}^{-2}$ (for the 11-2031 and CCDM J2137+5734 mini-clusters, the Tr 37 centre map), 1e+20-1.5e+21 $\mathrm{cm}^{-2}$ (for the $213911452 \mathrm{mini}-$ cluster and the $\operatorname{Tr} 37$ north map), and a $\log$ scale between $1 \mathrm{e}+20-1 \mathrm{e}+23 \mathrm{~cm}^{-2}$ (for the $\operatorname{Tr} 37$ west map). For the 11-2031 mini-cluster, $N_{\mathrm{H}}$ increases towards the centre of the filament (centre of the image). For CCDM J2137+5734, $N_{\mathrm{H}}$ increases towards the south-west, as the mini-cluster itself does not present any detectable overdensity with respect to the local background. Confirmed cluster members (with and without disks) are marked with stars, X-ray sources consistent with YSO are marked withs crosses. The O6.5 star HD 206267 is marked as a large magenta star in the cluster centre. Noise-dominated regions are excluded from the plots. Note that the temperature/column density derivation is only valid in regions that are optically thin, and thus not relevant in very dense parts or in pixels dominated by stellar emission. 


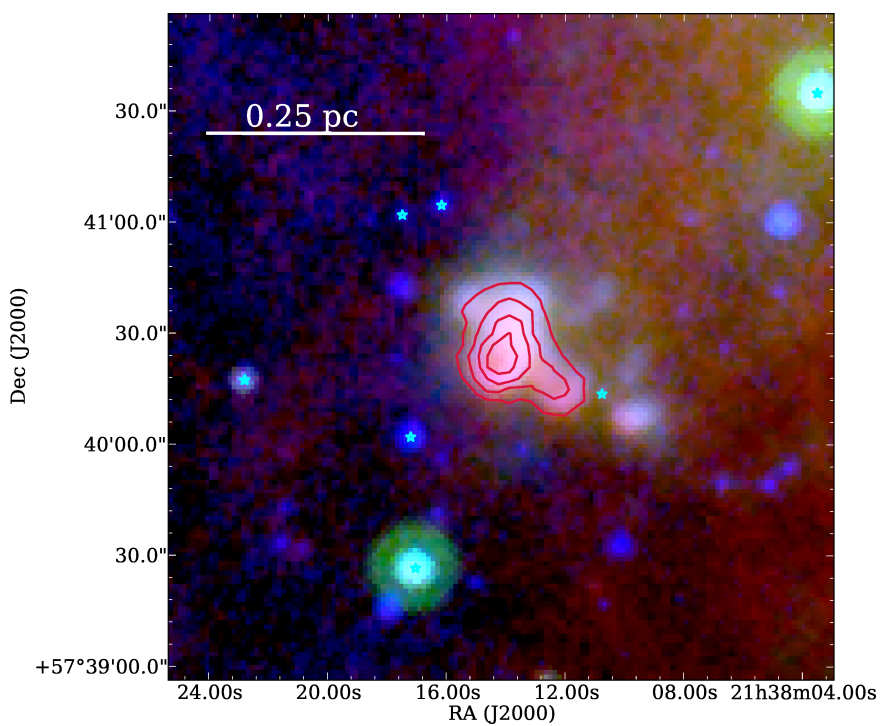

Fig. 8. Bright outflow-like object, consistent with a planetary nebula, located to the north of $\operatorname{Tr} 37$, as observed at $8 \mu \mathrm{m}$ (blue) $24 \mu \mathrm{m}$ (green), and $70 \mu \mathrm{m}$ (red), with $160 \mu \mathrm{m}$ shown as crimson contours.

detections and upper limits (see Appendix C for details). Stringent upper limits also confirm the status of disks classified as depleted/settled based on Spitzer data. The agreement between the Spitzer SED model predictions and Herschel observations suggests that our interpretation of disks in terms of evolved objects with large grains and relatively low mass in small dust grains is correct. Herschel/PACS also reveals interesting features in some individual objects, described in the following paragraphs.

\subsubsection{GM Cep: A rare intermediate-mass star in the making}

The G8 star GMCep is the brightest disk and the most conspicuous point source observed with Herschel/PACS in Tr 37. GMCep has a high and variable accretion rate $\left(\sim 10^{-7}-10^{-6} M_{\odot} / \mathrm{yr}\right)$, variable extinction, and the strongest $1.3 \mathrm{~mm}$ flux in the region (Sicilia-Aguilar et al. 2008, 2011; Semkov \& Peneva 2012). From its spectral type and high luminosity, it is probably the precursor of an A or B star, although it is very different at optical and IR wavelengths from other HAeBe stars, such as MVA-426 in Tr 37. The Herschel/PACS data confirm that the disk around GM Cep is more massive than the disk of the B7 star MVA-426 and the disks around late-type stars. GM Cep is located towards the cluster centre, in a region where most of the surrounding stars have mean ages around $4 \mathrm{Myr}$. If GMCep is a A/B star in the making, it would either have an anomalous age among the surrounding stars or a very longlived disk, being a key object to study the evolutionary paths of intermediate-mass stars. There is a significant difference between the $70 \mu \mathrm{m}$ excess measured with Spitzer/MIPS and the Herschel results, but given the MIPS non-linearities (Paladini et al. 2013) and the agreement between non-simultaneous IRAS, IRAC, MIPS, and IRS data, it cannot be attributed to variability.

\subsubsection{Low far-IR flux in a strongly accreting star: 11-2037}

The K4.5 star 11-2037 has a remarkable low $70 \mu \mathrm{m}$ flux for its Spitzer fluxes and its IRAM/1.3 mm detection (Sicilia-Aguilar et al. 2011). This is an example where our previous disk models fail to predict the observed Herschel fluxes, which are lower

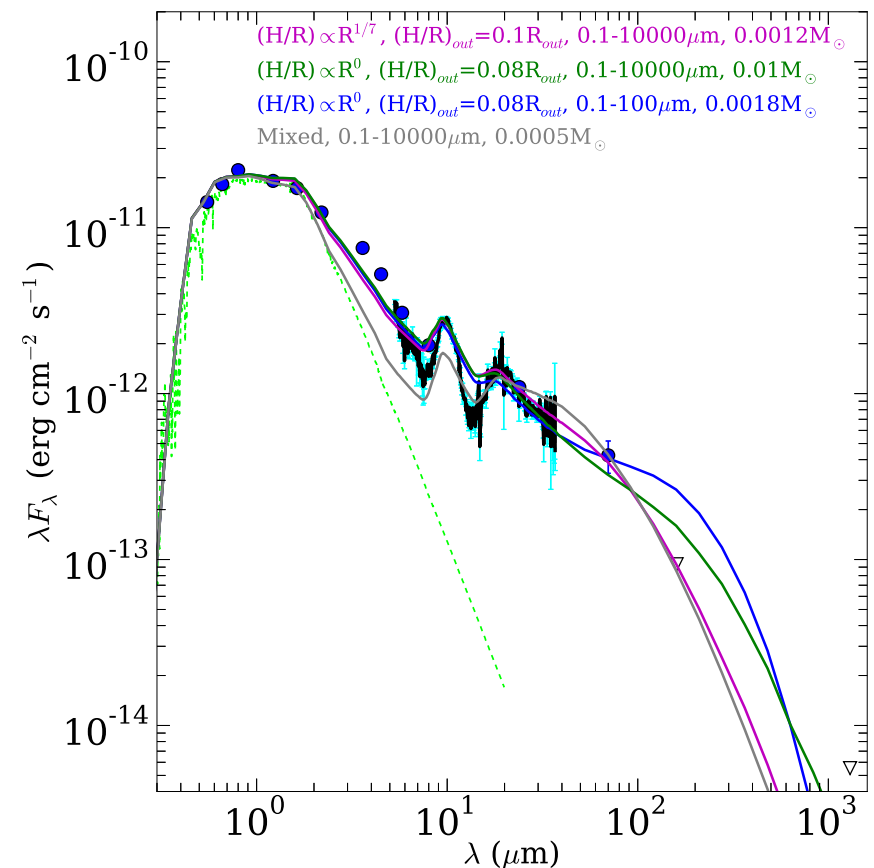

Fig. 9. Comparison of radiative transfer models for the disk of 01-580, the only accreting star in NGC 7160. Blue dots mark the data (errors are often smaller than the dots). Inverted triangles are upper limits. The dashed line is a MARCS photospheric model (Gustafsson et al. 2008). The colour lines show models with different vertical structures (flaring laws $H / R$, outer disk thickness), grain size distributions, and disk masses. The model labelled "Mixed" includes well-mixed dust and gas. Even if the disk is assumed to be very settled, substantial mass depletion is required to fit the $160 \mu \mathrm{m}$ upper limit.

than expected. The object is part of a mini-cluster (see Sect. 4.2), so nebular emission could have led to the contamination of the millimetre flux (and maybe of the $160 \mu \mathrm{m}$ flux as well), but the sharp turn-down at $70 \mu \mathrm{m}$ is real: although the source is one of the brighter sources compared to neighbouring disks at Spitzer wavelengths, it is clearly fainter at $70 \mu \mathrm{m}$. The sharp decline in $70 \mu \mathrm{m}$ emission could be due to a lack of dust, to strong settling/flattening at longer wavelengths, or to radially variable structures, such as wide gaps in the disk at larger distances. Further data (in particular, confirmation of the millimetre flux) is needed to test this hypothesis. If the dust mass is in fact lower than in other systems, it would offer a very strong contrast with the expected gas mass from accretion, since the object also has strong $\mathrm{H} \alpha$ emission and a high accretion rate $\left(\sim 10^{-8} M_{\odot} / \mathrm{yr}\right.$; Sicilia-Aguilar et al. 2005).

\subsubsection{Young in the gas, evolved in the dust: a survivor in NGC 7160}

The only object detected at $70 \mu \mathrm{m}$ in NGC 7160 is the $\mathrm{K} 4.5$ star 01-580. It is also the only disk with confirmed accretion in this 12 Myr-old cluster, and one of the strongest accretors among low-mass stars in the whole Cep OB2 region $(\dot{M}=$ $4_{-2}^{+3} \times 10^{-8} M_{\odot} / y r$; Sicilia-Aguilar et al. 2010). Remarkably, its mid-IR flux is relatively modest compared to similar objects in $\operatorname{Tr} 37$, which had been previously interpreted as a lack of small dust in the disk (Sicilia-Aguilar et al. 2011). The object is clearly detected at $70 \mu \mathrm{m}$, but not at $160 \mu \mathrm{m}$.

We run several models to reproduce its mid- and far-IR SED (Fig. 9). Low far-IR fluxes can result from dust settling, 
depletion of small-sized grains, and/or more dramatic shaping of the outer disk, such as very large gaps or external disk truncation. Given the high accretion rate, it is unlikely that the disk has very low gas mass, but the amount of mass in small dust could be low. Models assuming well-mixed gas and dust allow for a very small amount of dust mass, especially if the maximum grain size is $<100 \mu \mathrm{m}$. Flattening the disk allows us to increase the dust mass, but only as long as we still include substantial grain growth (beyond $\sim 100 \mu \mathrm{m}$ ) to reduce the $160 \mu \mathrm{m}$ excess. The best-fitting models to reproduce the observed midand far-IR SED are those with either settled disks with relatively low mass and strong grain growth, or flared disks with a reduced dust mass as well as strong grain growth.

Although the disk falls within our full-disk classification, its SED also betrays radial evolution, including a very strong near-IR excess (not well reproduced in our models and suggestive of a very thick inner disk or puffed inner wall) and a very strong silicate feature (similar to PTD, and thus suggestive of a non-uniform distribution of small grains). The object has thus signs of both inside-out evolution and more generalised dust evolution (strong grain growth, setting, maybe depending on radius, and/or depletion). The disk would also be a good candidate for anomalous gas-to-dust ratio (which would allow the disk to have a large gas mass despite having a reduced dust content), or dust filtration to the innermost part of the disk, as has been predicted (Hughes \& Armitage 2012) and suggested (Hughes et al. 2008) for other evolved objects.

These features are not shared with strong accretors in $\operatorname{Tr} 37$, which are typically small-grain rich. Old-accreting TTS are also often found to be relatively primordial, compared to what we observe in 01-580 (e.g. Ingleby et al. 2014). This suggests that at the old age of NGC 7160, even if the accretion behaviour is similar to that of younger disks, dust evolution has become unavoidable, as expected from models (Testi et al. 2014). Tr 37 also contains a few objects with steep mid- and far-IR SEDs (e.g. 111209 and 13-236 in Fig. C.1). Nevertheless, these objects have two significant differences compared to 01-580: They have very weak silicate features (negligible for 11-1209) and lower accretion rates $\left(\sim 5-6 \times 10^{-9} M_{\odot} / \mathrm{yr}\right)$. The best-fitting models for these objects require settled disks at all radial distances, which is not the case for 01-580. This makes 01-580 stand up as a remarkably active, old protoplanetary disk, and not simply as the last survivor among average protoplanetary disks.

\section{Discussion}

\subsection{Disk evolution in the light of Herschel data}

\subsubsection{The dust and gas connection}

Exploring the far-IR properties and accretion together helps to understand the connection between gas and dust in protoplanetary disks. For most of the stars, we have constraints on accretion (from high-resolution $\mathrm{H} \alpha$ observations; SiciliaAguilar et al. 2006b) and accretion measurements and upper limits (from $U$ band; Sicilia-Aguilar et al. 2005, 2010). Based on Sicilia-Aguilar et al. (2010), we also consider an upper limit of $10^{-11} M_{\odot} / \mathrm{yr}$ for objects with narrow $\mathrm{H} \alpha$ detected in high-resolution spectroscopy (Sicilia-Aguilar et al. 2006b). $\mathrm{H} \alpha$ strengths and profiles for such objects are indistinguishable from those of diskless TTS, and thus consistent with no accretion, although $U$ band measurements are limited to accretion rates over $\sim 10^{-11} M_{\odot} / \mathrm{yr}$ for late $\mathrm{K}$ and M stars ${ }^{8}$ (Sicilia-Aguilar et al. 2010). Accretion rates derived from other methods (e.g. $\mathrm{H} \alpha$ photometry or spectroscopy) are not considered since they show more scatter than $U$-band based measurements.

None of the non-accreting disks around low-mass stars is clearly detected with Herschel. The K2.5 star $213655283+5725516683$ is well-detected and has a large hole and possibly no accretion, although a high-resolution spectrum would be needed to confirm this. Another three non-accreting TD are consistent with marginal detections (73$758,13-350,21384350.5727270)$, although among those only 13-350 is clearly non-accreting. Disks without signs of accretion are rare and belong to the class of TD with inner holes, of which approximately half are consistent with no accretion, or to the low-excess/dust-depleted/settled SEDs (Sicilia-Aguilar et al. 2010; SA13). All full and PTD disks in Tr 37 have accretion signatures, even though the accretion rates range between a few times $10^{-10} M_{\odot} / \mathrm{yr}$ and $\sim 10^{-7} M_{\odot} / \mathrm{yr}$. Since the detectability of the disks around similar-type stars depends mostly on the disk mass and the flaring/thickness, this is a strong sign that disks with no accretion are either less massive and/or significantly settled compared to full disks. Different grain properties, such as very strong and global grain growth, could also reduce the far-IR emission in non-accreting disks, although most of them have evidence of small grains in their inner disks as shown by Spitzer IRS spectra (Sicilia-Aguilar et al. 2007, 2011).

Figure 10 displays the accretion rate $\dot{M}$ against different spectral indices for objects with various SED types. Interestingly, full disks appear clearly separated from transitional and pre-transitional disks. A double-sided KolmogorovSmirnov (KS) test reveals a probability of $0.002(0.2 \%)$ that the $\alpha(\mathrm{H}-70)$ of the PTD and TD are drawn from the same distribution as full disks, which is highly significant since the number of PTD and TD is small. In the case of $\alpha(8-70)$, the probability drops to $0.01 \%$, although this could be due to inner holes or gaps decreasing the continuum levels at $8 \mu \mathrm{m}: \alpha(8-70 \mu \mathrm{m})$ increases when the disk becomes more "transitional". In addition, for a given mass, flattening and/or settling in the disk reduce the difference between the 24 and $70 \mu \mathrm{m}$ flux. This can be observed in $\alpha(24-70 \mu \mathrm{m})$, although this index shows a stronger degeneracy.

Accreting TD and PTD in $\operatorname{Tr} 37$ have moderate but not significantly different accretion rates, compared to full disks, since very strong accretors are rare in $\operatorname{Tr} 37$ and generally correspond to stars with earlier spectral types (Sicilia-Aguilar et al. 2010). The difference becomes significant once a proxy for disk mass and/or flaring, such as the SED slope between the near- and farIR, is included. For the same accretion rate, PTD and TD tend to have higher $\alpha(\mathrm{H}-70 \mu \mathrm{m})$ spectral index than full disks. Imposing the condition of having similar accretion rates, the KS test produces probabilities of observing the same differences from samples drawn for the same distribution of $6 \%$ (for $\alpha(\mathrm{H}-24)$, not conclusive), $0.06 \%$ (for $\alpha(\mathrm{H}-70)$, highly significant), $0.08 \%$ (for $\alpha(8-70)$, highly significant), and $0.8 \%$ (for $\alpha(24-70)$, also significant). If we run the KS test on the spectral indices derived from our models instead, we find no significant differences between PTD/TD disks and full disks in any of the mentioned

\footnotetext{
8 As noted in Sicilia-Aguilar et al.(2010), the gap between nonaccreting objects and our accretion measurements, with nearly no objects with rates below a few times $10^{-10} M_{\odot} / y r$, is real and not a result of observational limitations. Accreting objects with upper limits to their $U$-band accretion rates usually have uncertain spectral types, anomalous extinction values, and/or larger photometry errors. Their $\mathrm{H} \alpha$ widths, profiles, and EW are fully consistent with typical CTTS, and not indicative of particularly low accretion rates.
} 

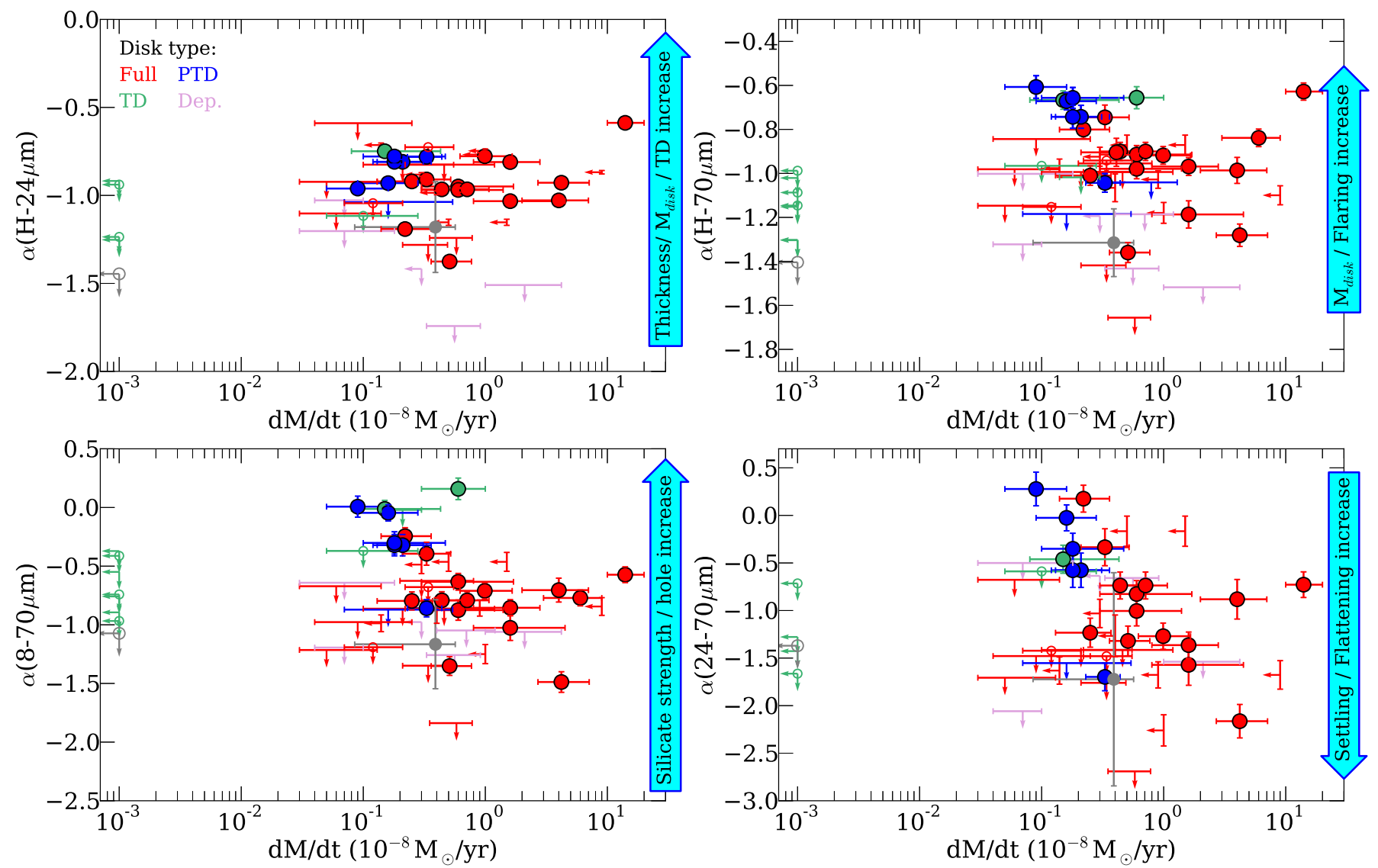

Fig. 10. The spectral indices $\alpha$ involving far-IR colours vs. accretion rates for stars with different disk types, classified according to their Spitzer colours (see text). Full disks are marked in red, PTD are marked in blue, TD are marked in green, and low excess/depleted disks are marked in pink. Circles represent detections (in both photometry and accretion rate), and upper limits in the accretion rate or spectral indices are marked by arrows. Marginal detections are marked with arrows, but we also show an open circle at the marginal value. The solid grey dot corresponds to the median value obtained by stacking the images of the non-detected objects. Its errorbars represent the dispersion in the $\alpha$ index and the quartiles in the accretion rate. The open grey dot is the median value (marginal detection) obtained by stacking the non-accreting disks. On the right, blue arrows show how certain changes in the disk properties would affect the observed spectral indices, as explored in Sect. 3.2.

spectral indices. The presence of a hole or a radial variation in disk properties per se is not enough to produce the observed difference if the remaining disk properties (disk mass, flaring, disk thickness, global dust grain properties) are kept the same for full and PTD/TD disks, even though vertically extended structures in the disks can alter the irradiation at different distances, causing a redistribution of flux towards longer wavelengths (e.g. Varnière et al. 2006). If disks with and without gaps are assumed to have well-mixed dust and gas in hydrostatic equilibrium, the difference between those with holes and without them is negligible for the same disk mass (see Fig. 3), as long as the holes are small. Therefore, we find that disks identified with Spitzer as candidates that have inner holes and/or gaps differ in their global radial and/or vertical structure with respect to objects classified as full disks. What happens in the inner disk is thus not completely disconnected from deeper changes in the global disk structure: observing systematically higher $70 \mu \mathrm{m}$ fluxes for TD and PTD is an indication that they have more massive and/or more flared/thicker disks, compared to full disks.

Disks with inner holes and gaps are good candidates for active giant planet formation. Massive planets, capable of clearing the disk, can change the disk vertical structure, especially in the inner rim close to the planets (e.g. Kley 1999). Changing the global disk flaring would require more dramatic mechanisms such as radially-variable settling (e.g., depending on density; Dubrulle et al. 1995). Planet-induced gaps can also produce radial variations in the dust properties due to dust filtering (Rice et al. 2006; Zhu et al. 2012). Accretion rates in the presence of giant planets are expected to be lower than what would correspond from viscous evolution for the total disk mass (Varnière et al. 2006; Lubow \& D'Angelo 2006), which is in agreement with previous observations of TD (Najita et al. 2007). Nevertheless, this depends on the ability of the planet to block the gas flow (and not only dust) through the gap, which is uncertain (Müller \& Kley 2013; Zhu \& Stone 2014) and inconsistent with some of the accreting binary systems known to date (e.g. Fang et al. 2014), including cases such as 82-272 in $\operatorname{Tr} 37$, which is an accreting spectroscopic binary (Sicilia-Aguilar et al. 2006b).

If the main cause of the difference in the far-IR slopes was the diversity of disk masses (as it happens in viscous accretion models; Hartmann et al. 1998), we would expect to observe a trend between the $\alpha(\mathrm{H}-70 \mu \mathrm{m})$ indices of the full disks and their accretion rates. This is not observed at significant levels. Variations in the disk vertical structure (flaring/thickness) are common in disks (Sicilia-Aguilar et al. 2011) and can affect $\alpha(\mathrm{H}-70 \mu \mathrm{m})$, washing out potential correlations between the $70 \mu \mathrm{m}$ fluxes and the accretion rate. There is also the possibility that the various physical conditions (or grain properties) throughout the disk affect the viscosity and thus the global viscous transport (Isella et al. 2009). In addition, the accretion rate baseline for detected objects is small, although the lack of 
detections of non-accreting disks points to a correlation between the $70 \mu \mathrm{m}$ flux and the mass of the disk.

Since many of the objects with low accretion are undetected at $70 \mu \mathrm{m}$, we followed Andrews \& Williams (2007) and stacked the non-detection images of the 16 disks with measured accretion rates (excluding objects near IC 1396 A that have complex background). This results in a $4.6 \sigma$ detection at $70 \mu \mathrm{m}$ $(3.7 \pm 0.8 \mathrm{mJy})$. We then estimate the median indices to compare with the median accretion rate $\left(3.9 \times 10^{-9} M_{\odot} / \mathrm{yr}\right.$, quartiles $\left.5.1 \times 10^{-9} M_{\odot} / \mathrm{yr}-8.5 \times 10^{-10} M_{\odot} / \mathrm{yr}\right)$. The median indices are consistent with steeper slopes for objects with lower accretion rates, although they do not make the accretion-spectral index correlation significant. They also show that non-detected disks accreting at low rates have small, but not tiny (comparable to other detected disks), mean dust masses. If we repeat the exercise for the eight confirmed (via high-resolution spectroscopy) non-accreting disks, including marginal detections, we obtain a marginal $3 \sigma$ detection of $2 \mathrm{mJy}$. The resulting spectral indices are consistent with strong disk evolution, although half of them are not detected at $24 \mu \mathrm{m}$ either. This provides further evidence that the mean disk mass for non-accreting disks is significantly lower than for accreting sources.

\subsubsection{Global disk evolution and the disk dispersal mechanisms}

Herschel far-IR observations confirm the existence of various distinct evolutionary paths in protoplanetary disks, likely related to the diversity of physical processes acting on disk removal and on the initial conditions. Having low dust masses or strongly settled disks as observed in dust-depleted objects does not trigger the immediate opening of a hole. Conversely, opening a hole or gap does not require a previous depletion of disk mass, at least in the case of accreting TD/PTD. In addition, shutting down the accretion of a disk seems only possible in case the disk mass has already decreased sufficiently. This probably requires dramatic changes in the whole disk, which would explain why the only non-accreting disks in Tr 37 are either TD or dust-depleted.

Different physical processes appear to be behind accreting and non-accreting disks, as previously suggested (SiciliaAguilar et al. 2007, 2010, 2011). Herschel data confirm the dichotomy in the transition disk classification. Non-accreting TD, despite having similar Spitzer colours than accreting TD (Fig. 10; Sicilia-Aguilar et al. 2011; SA13), are clearly separated by the spectral indices involving $70 \mu \mathrm{m}$, and their lower mean $70 \mu \mathrm{m}$ excesses are consistent with lower disk masses and/or lower flaring. Photoevaporation is a good candidate to explain the lack of near-IR excesses together with the lack of accretion signatures in non-accreting TD. Photoevaporation can open inner holes and shut down the accretion flow in a short timescale, as long as viscous transport does not replenish the inner disk at a faster rate (Clarke et al. 2001; Alexander et al. 2006; Gorti et al. 2009). Once the mass of the disk and the viscous transport rate have decreased below a certain limit, photoevaporation would take over shutting down accretion and eventually removing the rest of the disk. This theoretical picture is in agreement with the lack of disks accreting at very low rates (Sicilia-Aguilar et al. 2010), although the lack of correlation between the accretion rate and the far-IR indices suggests that the problem is more complex.

None of the objects classified as low excess/dust-depleted disks is detected with Herschel, which is also a strong sign that these objects are subject to substantial mass depletion or deep structural differences. The upper limits in Figs. 4 and 10 are
Table 4. Stars associated with the CCDM J2137+5734 mini-cluster.

\begin{tabular}{lccc}
\hline \hline Name & Sp. type & Disk type & $A_{V}(\mathrm{mag})$ \\
\hline CCDM J2137+5734e & B3 & Db & 1.7 \\
CCDM J2137+5734w & B5 & Db & 1.5 \\
$21374275+5733250$ & F9 & F & 1.6 \\
$21374376+5533169$ & K/M & N & 1.6 \\
$213744131+573331130$ & K7/M1 & F & 1.9 \\
$213742167+573431486$ & M2.0 & TD & 1.4 \\
$213740471+573433203$ & M2.5 & TD & 2.0 \\
\hline
\end{tabular}

Notes. Members of the CCDM J2137+5734 mini-cluster, an irregularshaped stellar grouplet. Spectral types and extinctions, from Contreras et al. (2002) and Sicilia-Aguilar et al. (2005, 2013a,b). There are no. individual age estimates for these objects. Disk classes according to Table 2, Db stands for debris disk candidate

consistent with depleted disks occupying a different parameter space than full disks. Strong settling at large radii or generalised depletion of small dust grains by grain growth could explain these observations. Unfortunately, the lack of detections among this class does not let us explore in detail the causes of the depletion.

\subsection{Multi-episodic star formation in Tr 37: mini-clusters}

Previous Spitzer studies revealed diffuse nebulosity around some grouplets of stars (G12; SA13). Herschel data offer a new view of the Tr 37 grouplets or mini-clusters (Fig. 6). The PACS data reveal the presence of cloud structures in the most remarkable mini-cluster, containing the star 11-2031. The dense structure next to the emission-line star 213911452+572425205 (SA13) is also detected with Herschel. Not all mini-clusters are detected at the same wavelengths, revealing differences in density and temperature. The following paragraphs discuss the morphology and properties of the $\operatorname{Tr} 37$ mini-clusters and their potential origin.

\subsubsection{Irregularly-shaped mini-clusters: the CCDM J2137+5734 grouplet}

The most conspicuous Spitzer mini-cluster, associated with the massive binary star CCDM J2137+5734 and with remarkable $24 \mu \mathrm{m}$ extended emission (B11, G12, SA13), does not show extended emission at PACS wavelengths. It contains at least five confirmed members, several of them detected at $70 \mu \mathrm{m}$. There is a large variety of SED types among its members (Table 4), including the diskless X-ray candidate $21374376+5733169$ (G12), and TD such as $213740471+573433203$ at the tip of the cloudlet (with strong $24 \mu \mathrm{m}$ emission) and $213742167+573431486$. The IR excess of the second could be affected by nebular contamination, even though it shows strong $\mathrm{H} \alpha$ emission from accretion.

This structure has the highest temperature and likely the lowest density among all the mini-clusters. Herschel-based estimates of temperature and column density are not substantially different from the surrounding background (local background $N_{\mathrm{H}} \sim 1 \times 10^{20} \mathrm{~cm}^{-2}$ ). Although heating in $\operatorname{Tr} 37$ is dominated by the central O6.5 star, the two B stars CCDM J2137+5734A/B may affect the temperature and dispersal rate of the cloudlet at shorter distances, leading to a thinner, warmer structure compared to other mini-clusters only populated by low-mass stars. This is comparable to the findings of Roccatagliata et al. (2013), although at a much smaller scale. 
Table 5. Stars associated with the 11-2037/11-2031 mini-cluster.

\begin{tabular}{lccc}
\hline \hline Name & Sp. type & Age $(\mathrm{Myr})$ & $A_{V}(\mathrm{mag})$ \\
\hline $11-2037$ & K4.5 & 2.5 & 1.6 \\
$11-2131$ & K6.5 & 3.0 & 2.3 \\
$21371389+5727270^{*}$ & late K & - & 3.0 \\
$21371545+5727170^{*}$ & K/M & - & 2.3 \\
$11-2031$ & K2.0 & 4.5 & 1.7 \\
$213716349+57264020$ & K7.0 & - & $6.0:$ \\
$21372152+5726123^{*}$ & K/M & - & 1.7 \\
\hline
\end{tabular}

Notes. Members of the 11-2037/11-2031 mini-cluster, a stellar grouplet with a filamentary structure and associated nebulosity. Spectral types, extinctions, and ages from Sicilia-Aguilar et al. (2005, 2013a,b), except for objects marked with *, for which they are derived in the present work from SED fitting. All SEDs are consistent with "full disks".

\subsubsection{Filamentary mini-clusters: the 11-2031 grouplet}

The 11-2031 mini-cluster has the shape of an elongated filament with six confirmed young stars, plus a potential further member with $24 \mu \mathrm{m}$ emission. The star $21370909+5725485$ is located at a similar distance as 11-2037, but since it is not surrounded by the same patch of nebulosity, it may not belong to the group. All the mini-cluster members have similar spectral types and masses around $1 M_{\odot}$ (considering the evolutionary tracks of Siess et al. 2000 for a 3-4 Myr age), and they all have full disks (Table 5). There is a tendency to find younger cluster members towards the west of $\operatorname{Tr} 37$ (Sicilia-Aguilar et al. 2005), but the mini-cluster members are not significantly younger than the main cluster, considering the individual uncertainties. Radial velocities available for two of the stars $\left(11-2037 \mathrm{cz}=-19.9 \pm 0.6 \mathrm{~km} \mathrm{~s}^{-1}\right.$; $\left.11-2031 \mathrm{cz}=-17.2 \pm 1.2 \mathrm{~km} \mathrm{~s}^{-1}\right)$ are consistent with the average cluster value $\left(-15.2 \pm 3.6 \mathrm{~km} \mathrm{~s}^{-1}\right.$; Sicilia-Aguilar et al. 2006b). But the SED types for the mini-cluster members are clearly different from the bulk of $\operatorname{Tr} 37$ members: they all have full disks. Since the disk fraction in $\operatorname{Tr} 37$ is $\sim 48 \%$, and nearly $1 / 3$ of stars have disks classified as TD, PTD, or low-excess/mass depleted, the probability of finding seven full disks among near neighbours by chance is below $0.1 \%$.

Herschel data reveals column density enhancements over the background in the range $\sim 2-7 \times 10^{19} \mathrm{~cm}^{-2}$ (background levels are $\sim 2 \times 10^{20} \mathrm{~cm}^{-2}$ at this location). Integrating over the observed structure, we estimate a total mass of $\sim 0.05 M_{\odot}$ in nebular material. This would be similar to the mass of a few protoplanetary disks around low-mass stars, albeit distributed over a very large area $\left(\sim 0.036 \mathrm{pc}^{2}\right)$. Since the filament contains six (maybe seven) low-mass stars with disks, the mass in the remnant cloud is of the order of the total mass in the protoplanetary disks. The cloud is optically thin and relatively warm, unlikely to be infalling onto the objects, even though the filamentary nebulosity probably contains remnant material from the initial core. The extinction towards the mini-cluster members is not significantly higher than the cluster average $\left(A_{V}=1.67 \pm 0.45\right.$; Sicilia-Aguilar et al. 2005), consistent with excesses around 0.1-0.2 mag expected from the estimated column density. Only $213716349+572640200$ is significantly more extincted than the rest, which could be due to disk orientation or to the presence of remnant envelope material (which could explain the brightness of the object at $160 \mu \mathrm{m})$.

The stars are separated by less than $0.1 \mathrm{pc}$ projected distance, with $11-2037$ at $\sim 0.2 \mathrm{pc}$ from the rest. The star $21372152+5726123$ is also located at $0.2 \mathrm{pc}$ of $213716349+57264020$, but if the $24 \mu \mathrm{m}$ excess source at
$21: 37: 18.9+57: 26: 25.7$ could be confirmed as a young star, the separation would also be around $0.1 \mathrm{pc}$. This is very similar to the beads-on-a-string picture predicted for gravitational fragmentation of a thin sheet or filament (Hartmann 2002; WardThompson et al. 2010; Nielbock et al. 2012). Here, the filament is much smaller in size than the usual structures, and the masses of the individual "beads" are also low $\left(\sim 1 M_{\odot}\right)$. In the simple model for an infinite cylinder of Heitsch et al. (2009), the critical mass for a scale of $0.1 \mathrm{pc}$ is similar to the masses of the stars in the mini-cluster, although this would imply an unrealistic star formation efficiency. By itself, such a low mass filament would be not form stars unless shielded from external radiation by further cloud material. One possibility of forming such a small structure would be gravitational focusing in the collapse of an asymmetric sheet (Burkert \& Hartmann 2004), which tends to concentrate material towards the edges of the collapsing finite structure. In this case, it would be a sign that gravitational fragmentation operates in similar ways in spatial scales from fractions of a pc up to several hundred pc.

\subsubsection{Other cloud structures in $\operatorname{Tr} 37$}

The third mini-cluster contains the M1 star 213911452+ 572425205 (which has a full disk) and the probably diskless K6.5 star 213905519+572349596, being the smallest grouplet and thus the less representative. Unlike in the other miniclusters, both stars are at the rim of the extended nebular structure. The star $213911452+572425205$ has a massive, young disk, with strong accretion and remarkable emission lines (SA13). The globule, clearly detected at Spitzer and Herschel wavelengths, shows a remarkable dense and cold centre. Our column density estimates are in the range $\sim 1.0-17 \times 10^{20} \mathrm{~cm}^{-2}$, giving an approximate mass $\sim 0.15 M_{\odot}$ integrating over the globule surface. This is comparable to the mass of a very low-mass star, but it is unlikely to be cold and dense enough to undergo gravitational collapse. The globule has a very steep density gradient, in contrast with the other two mini-clusters, which merge smoothly with the cluster background. There is no evidence of shock lines that suggest interaction between the young stars and the nebula, so chance projection is also a possibility. If so, this globule could be a starless structure, maybe a very low mass, but dense, clump left over of the removal of the original cloud.

Further small patches of nebulosity are found throughout $\operatorname{Tr} 37$, especially at $160 \mu \mathrm{m}$. Several structures are seen towards the west of $\operatorname{Tr} 37$, behind the IC 1396 A ionization front (Fig. 7). They have rounded shapes and sizes ranging from several tens of arcsec to 1 arcmin. Some smaller, rounded structures are also visible through the cluster, but since they are typically faint at Herschel wavelengths, it is not possible to determine accurate temperatures or column densities. A few individual sources seem to be associated with the nebular structures (e.g. 12-1091). Nevertheless, given the abundance of small nebulosity patches not associated with stellar sources, there is a high risk that they result from chance projection.

\subsubsection{The star-formation history of $\operatorname{Tr} 37$}

The diversity in stellar content shows that the differences in the mini-clusters properties do not correspond to time evolution within the same class of objects, but to essentially different grouplets, which may have formed from clouds with different properties and/or various triggering mechanisms, suggestive of a complex star-formation history in Tr 37. Paper I 
revealed multi-episodic star formation triggered by the O6.5 star HD 206267 in IC 1396 A (Paper I). Herschel/PACS observations show an arc-shaped structure about $0.5 \mathrm{pc}$ in length, which contains at least one intermediate-mass Class 0 object most likely triggered by radiation-driven implosion (RDI). Formation and collapse of a dense structure that was initially inside a larger cloud is thus possible. The similar-mass, similar-separation observed in the stars in the 11-2031 mini-cluster are more consistent with gravitational fragmentation, but the mini-cluster associated with the binary B3+B5 star CCDM J2137+5734 shows a very different morphology and stellar content. Besides the intermediate-mass binary, the mini-cluster contains a further relatively massive source $(21374275+5733250$, spectral type F9) at $\sim 16^{\prime \prime}$ separation, and some low-mass stars. It could thus have resulted from a similar structure as observed at the tip of IC $1396 \mathrm{~A}$, which is consistent with an intermediate-mass protostar or a small stellar grouplet containing intermediate-mass protostars.

The differences in the mini-clusters associated with 11-2031 and with CCDM J2137+5734 suggest that star formation in $\operatorname{Tr} 37$ has progressed in a multi-episodic way, similar to what we observe in the IC 1396 A globule. The mini-clusters are very compact structures $(\sim 0.25 \mathrm{pc}$ in size). Having clearly distinct grouplets of stars in a relatively old cluster such as $\operatorname{Tr} 37$ adds further evidence to the hypothesis that the objects in a minicluster originated within the same parental molecular core, as has been suggested for sub-clusters (Getman et al. 2014). Since the typical velocity dispersions in star-forming clouds are of the order of $1-2 \mathrm{~km} \mathrm{~s}^{-1}$ (consistent with our kinematic study of Tr 37; Sicilia-Aguilar et al. 2006b), objects could move about 1-2 pc in 1 Myr. This is incompatible with maintaining the mini-cluster structure over timescales similar to the ages of their members: even if part of the original members of the grouplet may have dispersed, the long survival of mini-clusters requires that their members formed together from a comoving filament or core with little velocity dispersion. Strong dynamical interactions between the grouplet members would also need to be prevented, as they would result in the dispersal of the mini-cluster members on short timescales (Bate 2012). The stellar and nebular content of the mini-clusters also shows that clumpy and multi-episodic star formation may proceed in various ways at small scales within the same cloud. Although at present we do not have velocity information for the mini-clusters, this could change our understanding of the star formation scenario in $\operatorname{Tr} 37$ and similar regions, suggesting that cloud fragmentation may proceed in an irregular way, with various small structures fragmenting and collapsing independently in time to give birth to small grouplets of stars.

\section{Summary and conclusions}

Herschel is a powerful tool to understand protoplanetary disks as a whole, revealing a strong connection between the innermost disk and outer/global disk evolution. The results of our Herschel survey on protoplanetary disks in the Cep OB2 clusters Tr 37 and NGC 7160 are summarised below:

- We detected 95 disks at $70 \mu \mathrm{m}$ and 41 at $160 \mu \mathrm{m}$, adding significant upper limits to more than 130 disks. The detection fraction is strongly dependent on the Spitzer SED type and the stellar spectral type. More than $50 \%$ of full disks and PTD are detected, while we obtain zero detections among low-excess/depleted disks. Nearly $90 \%$ of the disks around stars $\mathrm{K} 4$ or earlier are detected, compared to fewer than $1 / 3$ of the disks around M-type stars. Therefore, our results for $\mathrm{K}$ stars are representative for the whole disk class, while for M-type objects we only explore the most massive and flared disks.

- We find a large variety of disks among solar-type stars in $\operatorname{Tr} 37$. Far-IR data breaks the degeneracy of disk structures inferred from $24-30 \mu \mathrm{m}$ data, revealing details such as global settling/flattening, disk masses, and changes in the small-dust grain distribution. Herschel confirms structural differences behind the Spitzer-based classification in terms of full disks, disks with inside-out evolution (TD, PTD), and low-excess/dust-depleted disks. Low-excess/depleted objects are confirmed (via stringent upper limits) to be consistent with a low content of small dust grains and/or global mass depletion.

- The 70 and $160 \mu \mathrm{m}$ observations of disks in Tr 37 are consistent with strong dust grain growth, with maximum grain sizes over $100 \mu \mathrm{m}$. Even the most IR-luminous, massive and flared disks (expected to contain more pristine, less evolved grains than disks with low excesses) are consistent with substantial grain growth, suggesting that it is a rapid and universal process.

- None of the non-accreting disks is clearly detected with Herschel. Mean far-IR excesses for non-accreting disks are significantly lower than for accreting disks, which is consistent with non-accreting disks being more evolved, less massive and/or more settled disks.

- Herschel confirms the dichotomy of accreting vs. nonaccreting TD as objects resulting from different physical processes. Stopping accretion in a disk requires substantial changes to its global structure. All non-accreting disks are either TD or low-excess/depleted disks. Having a relatively massive and flared disk without accretion seems excedingly rare, so even objects with inner disk evolution (TD, PTD) are accreting if their disks are sufficiently massive. This provides strong evidence that accretion is mainly governed by the total mass content of the disk, as expected from viscous evolutionary models. It also suggests that photoevaporation is not efficient in opening a gap unless the disk mass has already decreased considerably.

- We observe significantly higher $\alpha(\mathrm{H}-70 \mu \mathrm{m})$ indices for PTD/TD, compared to full disks with similar accretion rates. Inner holes/gaps per se do not affect the $70 \mu \mathrm{m}$ flux much, compared to other parameters such as disk mass and thickness/flaring. One possibility could be that accreting TD and PTD disks are systematically more massive than full disks with similar accretion rates, in agreement with disk clearing by massive (planetary?) companions (Najita et al. 2007). Nevertheless, this would also predict a correlation between the accretion rate and the $\alpha(\mathrm{H}-70 \mu \mathrm{m})$ index for full disks, which is not observed. Another explanation could be the presence of higher vertical structures (walls, puffed-up rims) in PTD/TD shifting of the emission peak of the disk to longer wavelengths. This suggests that inside-out evolution and changes in the innermost disk are not disconnected from the outer- or global disk evolution.

- At old ages, significant dust processing/settling seems unavoidable, even if the object is still gas rich and strongly accreting. The only accreting disk in NGC 7160, 01-580, is a surprising case combining a very low far-IR flux with a remarkably high accretion rate. Studying such objects may be the key to understanding disk removal and survival. 
Herschel/PACS also reveal strong evidence for multi-episodic star formation in $\operatorname{Tr} 37$. Our Herschel observations allow us to conclude that:

- The extended cloud emission in Tr 37 contains several small structures or mini-clusters, compact $(\sim 0.25 \mathrm{pc})$ groups of young stars still surrounded by nebulosity. The physical properties of the mini-clusters (approximate dust temperature and column density) depend on the type of stars that they contain (e.g. intermediate-mass vs only solar-mass stars).

- Mini-clusters show that clumpy or multi-episodic star formation can occur even at relatively small scales of fractions of pc. Clumpy and multi-episodic star formation may also contribute to the variations in age and evolutionary stage observed between disks on relatively small spatial scales.

- The survival of the very compact mini-clusters after ages of 1-2 Myr indicates that their members were formed within the same cloudlet or core and without much internal disruption or interaction: Otherwise, the typical velocity dispersion of stars in clusters $\left(1-2 \mathrm{~km} \mathrm{~s}^{-1}\right)$ would have already disrupted them by the ages of $\operatorname{Tr} 37$.

- The mini-cluster associated with the binary B star CCDM J2137+5734 has the lowest density and highest temperature among the mini-clusters, and it could have originated in a structure similar to the IC 1396 A-PACS-1 protostar at the tip of the IC 1396 A globule.

- The mini-cluster associated with the low-mass star 11-2031 bears strong resemblance with a filament with equidistant, similar-mass stars (beads-on-a-string). With the total length of the filament being $\sim 0.5 \mathrm{pc}$, and the distance between the $\sim 1 M_{\odot}$ stars being less than $0.1 \mathrm{pc}$, it could be an example of gravitational fragmentation and/or gravitational focusing in a collapsing cloud/sheet down to very small scales.

- Herschel also reveals a bright structure to the north of $\operatorname{Tr} 37$, morphologically suggestive of a planetary nebula and most likely (given the cluster age) not associated with $\operatorname{Tr} 37$.

- Finally, Herschel observations reveal what could be one of the first episodes of heavy mass loss in the O6.5 star HD 206267, a sign of unmistakable evolution in the heart of $\operatorname{Tr} 37$.

Acknowledgements. We thank the anonymous referee for their comments that helped to improve and clarify this paper. We thank Bruno Altieri from the Herschel Helpdesk for his valuable help with the data reduction, Lorenzo Piazzo for making available the Unimap code, and Peter Woitke for the interesting discussion. We also thank Sofia Sayzhenkova from the computing support at the Departamento de Física Teórica, and Y. Ascasíbar, E. Villaver, and A. Díaz for their help identifying the field planetary nebula found in $\operatorname{Tr} 37$. A.S.A. acknowledges support by the Spanish MICINN/MINECO "Ramón y Cajal" program, grant number RYC-2010-06164. A.S.A. and M.F. acknowledge support by the action "Proyectos de Investigación fundamental no orientada", grant number AYA2012-35008. C.E. is partly supported by Spanish MICINN/MINECO grant AYA2011-26202. V.R. is supported by the DLR grant number 50 OR 1109 and by the Bayerischen Gleichstellungsförderung (BGF). T.B. acknowledges support from NASA Origins of Solar Systems grant NNX12AJ04G. This research has made use of the SIMBAD database, operated at CDS, Strasbourg, France. We also made use of Astropy, a community-developed core Python package for Astronomy (Astropy Collaboration, 2013) and APLpy, an open-source plotting package for Python hosted at http://aplpy.github.com.

\section{References}

Alexander, R., Clarke, C., \& Pringle, J., 2006, MNRAS, 369, 229 Andrews, S., \& Williams, J. 2007, ApJ, 671, 1800
Balog, Z., Rieke, G. H., Su, K. Y. L., Muzerolle, J., \& Young, E. T. 2006, ApJ, 650, L83

Bate, M. R. 2012, MNRAS, 419, 3115

Barentsen, G., Vink, J. S., Drew, J. E., et al. 2011, MNRAS, 415, 103 [B11]

Briceño, C., Vivas, A. K., Calvet, N., et al. 2001, Science, 291, 93

Burkert, A., \& Hartmann, L. 2004, ApJ, 616, 288

Clarke, C., Gendrin, A., \& Sotomayor, M. 2001, MNRAS, 328, 485

Contreras, M. E., Sicilia-Aguilar, A., Muzerolle, J., et al. 2002, AJ, 124, 1585

Cox, N. L. J., Kerschbaum, F., van Marle, A.-J., et al. 2012, A\&A, 537, A35

Currie, T., Lada, C., Plavchan, P., et al. 2009, ApJ, 698, 1

Cutri, R. M., Skrutskie, M. F., van Dyk, S., et al. 2003, VizieR On-line Data Catalog: II/246

D’Alessio, P., Calvet, N., Hartmann, L., Muzerolle, J., \& Sitko, M. 2004, Star Formation at High Angular Resolution, 221, 403

D’Alessio, P., Merín, B., Calvet, N., Hartmann, L., \& Montesinos, B. 2005, Rev. Mex. Astrophys. Astron., 41, 1

Dommanget, J. 1983, Bull. Inform. Centre de Données Stellaires, 24, 83

Dubrulle, B., Morfill, G., \& Sterzik, M. 1995, Icarus, 114, 237

Dullemond, C., \& Dominik, C. 2004, A\&A, 417, 159

Elias, N. M., II, Koch, R. H., \& Pfeiffer, R. J. 2008, A\&A, 489, 911

Fang, M., Sicilia-Aguilar, A., Roccatagliata, V., et al. 2014, A\&A, 570, A118

Getman, K. V., Feigelson, E. D., Sicilia-Aguilar, A., et al. 2012, MNRAS, 426, 2917 [G12]

Getman, K. V., Feigelson, E. D., Kuhn, M. A., et al. 2014, ApJ, 787, 108

Gorti, U., Dullemond, C. P., \& Hollenbach, D. 2009, ApJ, 705, 1237

Groenewegen, M. A. T., Waelkens, C., Barlow, M. J., et al. 2011, A\&A, 526, A162

Hartmann, L. 2002, ApJ, 578, 914

Heitsch, F., Ballesteros-Paredes, J., \& Hartmann, L. 2009, ApJ, 704, 1735

Herbst, W., Maley, J. A., \& Williams, E. C. 2000, AJ, 120, 349

Hernández, J., Hartmann, L., Megeath, S. T., et al. 2007, ApJ, 662, 1067

Howard, C. D., Sandell, G., Vacca, W. D., et al. 2013, ApJ, 776, 21

Hughes, A. L. H., \& Armitage, P. J. 2012, MNRAS, 423, 389

Hughes, A. M., Wilner, D. J., Qi, C., \& Hogerheijde, M. R. 2008, ApJ, 678, 1119

Ingleby, L., Calvet, N., Hernández, J., et al. 2014, ApJ, 790, 47

Isella, A., Carpenter, J. M., \& Sargent, A. I. 2009, ApJ, 701, 260

Kley, W. 1999, Two decades of Numerical Astrophysics, July 5-9 [arXiv: astro-ph/9909394]

Kuhn, M. A., Feigelson, E. D., Getman, K. V., et al. 2014, ApJ, 787, 107

Lubow, S. H., \& D'Angelo, G. 2006, ApJ, 641, 526

Mercer, E. P., Miller, J. M., Calvet, N., et al. 2009, AJ, 138, 7 [M09]

Morales-Calderón, M., Stauffer, J. R., Rebull, L., et al. 2009, ApJ, 702, 1507 [MC09]

Müller, T. W. A., \& Kley, W. 2013, A\&A, 560, A40

Najita, J., Strom, S., \& Muzerolle, J. 2007, MNRAS, 368, 379

Nielbock, M., Launhardt, R., Steinacker, J., et al. 2012, A\&A, 547, A11

Osterbrock, D. E. 1989, Astrophysics of gaseous nebulae and active galactic nuclei (University Science Books)

Ott, S. 2010, Astronomical Data Analysis Software and Systems XIX, ASP Conf. Ser., 434, 139

Paladini, R., Ali, B., Altieri, B., et al. 2013, PACS Map-making Tools: Analysis and Benchmarking, http://herschel.esac.esa.int/twiki/pub/ Public/PacsCalibrationWeb/pacs_mapmaking_report_ex_sum_v3. pdf

Patel, N. A., Goldsmith, P. F., Snell, R. L., Hezel, T., \& Xie, T. 1995, ApJ, 447, 721

Patel, N. A., Goldsmith, P. F., Heyer, M. H., \& Snell, R. L. 1998, ApJ, 507, 241

Peter, D., Feldt, M., Henning, T., \& Hormuth, F. 2012, A\&A, 538, A74

Piazzo, L. 2013 [arXiv: 1301.1246]

Piazzo, L., Ikhenaode, D., Natoli, P., et al. 2012, Artifact removal for GLS map makers by means of post-processing, IEEE Trans. on Image Processing, 21, 3687

Pilbratt, G. L., Riedinger, J. R., Passvogel, T., et al. 2010, A\&A, 518, L1

Platais, I., Kozhurina-Platais, V., \& van Leeuwen, F. 1998, AJ, 116, 2423

Poglitsch, A., Waelkens, C., Geis, N., et al. 2010, A\&A, 518, L2

Preibisch, T., Roccatagliata, V., Gaczkowski, B., \& Ratzka, T. 2012, A\&A, 541, A132

Rice, W., Armitage, P., Wood, K., \& Lodato, G. 2006, MNRAS, 373, 1619

Robitaille, T. P., Tollerud, E. J., Astropy Collaboration, et al. 2013, A\&A, 558, A33

Roccatagliata, V., Preibisch, T., Ratzka, T., \& Gaczkowski, B. 2013, A\&A, 554, A6

Semkov, E. H., \& Peneva, S. P. 2012, Ap\&SS, 338, 95

Sharpless, S. 1959, ApJS, 4, 257

Sicilia-Aguilar, A., Hartmann, L., Briceño, C., Muzerolle, J., \& Calvet, N. 2004, AJ, 128, 805 
Sicilia-Aguilar, A., Hartmann, L. W., Hernández, J., Briceño, C., \& Calvet, N. 2005, AJ, 130, 188

Sicilia-Aguilar, A., Hartmann, L., Calvet, N., et al. 2006a, ApJ, 638, 897

Sicilia-Aguilar, A., Hartmann, L., Fürész, G., et al. 2006b, AJ, 132, 2135

Sicilia-Aguilar, A., Hartmann, L., Watson, D., et al. 2007, ApJ, 659, 1637

Sicilia-Aguilar, A., Henning, Th., \& Hartmann, L. 2010, ApJ, 710, 597

Sicilia-Aguilar, A., Henning, T., Dullemond, C. P., et al. 2011, ApJ, 742, 39

Sicilia-Aguilar, A., Henning, T., Linz, H., et al. 2013a, A\&A, 551, A34

Sicilia-Aguilar, A., Kim, J. S., Sobolev, A., et al. 2013b, A\&A, 559, A29 [SA13]

Sicilia-Aguilar, A., Roccatagliata, V., Getman, K., et al. 2014, A\&A, 562, A131 (Paper I)

Siess, L., Dufour, E., \& Forestini, M. 2000, A\&A, 358, 593
Testi, L., Birnstiel, T., Ricci, L., et al. 2014, Protostars and Planets VI (University of Aizona Press), accepted [arXiv: 1402 . 1354]

Varnière, P., Bjorkman, J. E., Frank, A., et al. 2006, ApJ, 637, L125

Vamvatira-Nakou, C., Royer, P., Hutsemékers, D., et al. 2011, Bull. Soc. Roy. Sci. Liège, 80,435

Vamvatira-Nakou, C., Hutsemékers, D., Royer, P., et al. 2013, A\&A, 557, A20

Ward-Thompson, D., Kirk, J. M., André, P., et al. 2010, A\&A, 518, L92

Whitney, B. A., Robitaille, T. P., Bjorkman, J. E., et al. 2013, ApJS, 207, 30

Wright, E. L., Eisenhardt, P. R. M., Mainzer, A. K., et al. 2010, AJ, 140, 1868

Zhu, Z., \& Stone, J. M. 2014, ApJ, 795, 53

Zhu, Z., Nelson, R. P., Dong, R., Espaillat, C., \& Hartmann, L. 2012, ApJ, 755, 6 


\section{Appendix A: SEDs and photometry tables for all the YSO observed by Herschel}

This appendix contains full collection of SEDs of objects observed with Herschel, together with the complete Herschel photometry of the sources ${ }^{9}$. The first part (Table A.1, Figs. A.1 to A.3) includes the sources previously known and confirmed from our spectroscopic surveys (Sicilia-Aguilar et al. 2005, 2006b; SA13). The second part includes objects identified as YSO via Spitzer, $\mathrm{H} \alpha$ photometry, and/or X-ray observations (Table A.2, Fig. A.4). Table A.3 contains the upper limits for sources with disks identified via optical spectroscopy, whose SEDs are displayed in Figs. A.5-A.8. Table A.4 contains the upper limits for sources with disks from H $\alpha$ (B11), X-ray (M09; G12), and Spitzer (MC09) surveys, whose SEDs are displayed in Fig. A.9. Only sources with confirmed or potential IR excesses and significant upper limits (those that impose some contraints on the source SED) are considered. Most of the sources located within strong nebular emission in and near the IC 1396 A globule are excluded from the tables and figures, as their upper limits are typically several orders of magnitude higher than any reasonable value expected from their Spitzer data. A few objects with uncertain mid-IR excesses are also listed as upper limits.

The optical data for the sources was compiled from the existing literature (Sicilia-Aguilar et al. 2005, 2010; SA13; G12; B11; M09). Sources lacking this information in their original paper were searched for in our optical photometry databases, which are presented in detail in Sicilia-Aguilar et al. (2005; including VRI data from the Fred Lawrence Whipple Observatory) and SiciliaAguilar et al. (2010; with UVRI data from LAICA/3.5 m Calar Alto Telescope). For very bright sources such as HD 206267 and CCDM J2137+5734, the optical data was obtained from SIMBAD. The sources were also matched to the 2MASS catalog (Cutri et al. 2003) to obtain JHK photometry. The Spitzer data was compiled from the literature for the optical spectroscopy members (SA13). For the remaining candidates, we re-reduced the existing Spitzer data following the same procedures as in SA13, and also obtained the WISE counterparts from the WISE All-Sky Catalog (Wright et al. 2010) for objects without Spitzer data. All the plotted SEDs are corrected for the measured extinction, when available, or using the cluster average or best-fit extinction to the photospheric data of the star (if no measurement of $A_{V}$ is published).

The detection of photospheric fluxes at $70 \mu \mathrm{m}$ is limited to the O star HD 206267, which makes all upper limits on diskless intermediate- and low-mass stars irrelevant. Objects such as cold TD or debris disks (with excesses only at $70 \mu \mathrm{m}$ and beyond) around low-mass stars would typically remain undetected, so potential excesses around otherwise diskless objects must be handled with care, as they can easily be the result of contamination by cloud emission or background, unrelated objects.

Some of the disks are labelled as "marginal detections". These are cases where we observe spatially variable, patchy, sky background in the proximities of the source and with similar bightness as we would expect from source itself. This could be a sign of mismatch or contamination, even if visual inspection reveals the presence of a point-like source. For them, we consider that the flux of the object, even though more uncertain than in clear detections as it could be contaminated, is probably close to the observed "marginal detection". This distinguishes marginal detections from plain upper limits, where there is no hint of point source emission at the location of the source and thus the flux at the corresponding wavelength could in principle be much lower than the actual upper limits. For all other analysis throughout the paper, marginal detections are considered as upper limits.

\footnotetext{
9 Full photometry and spectroscopy, including optical and IR data, is available upon request to the first author.
} 


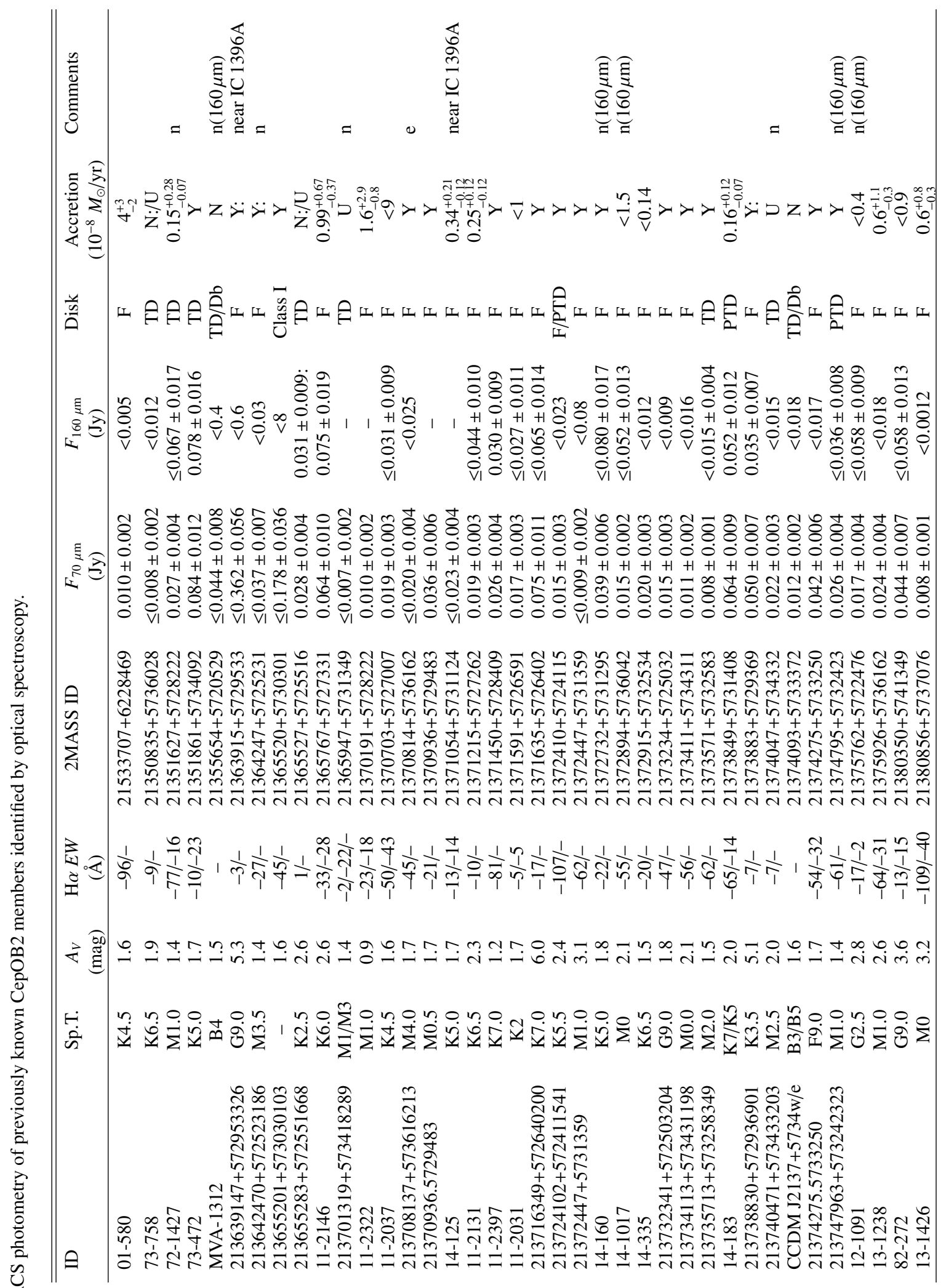

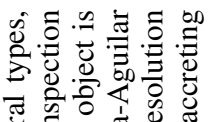

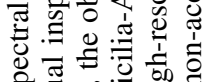

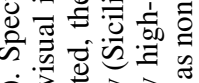

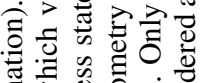

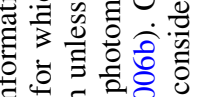
(

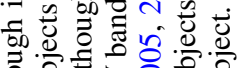

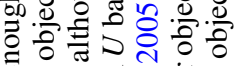
च छ

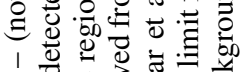

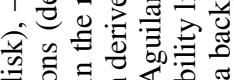

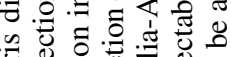
过 คิ

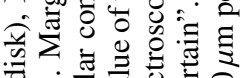

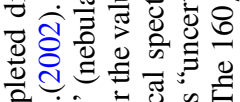

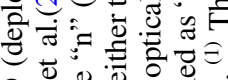
可 苛

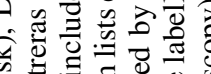

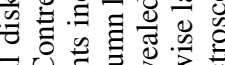
등

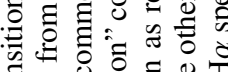

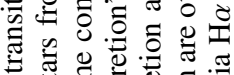

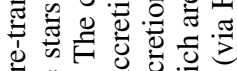

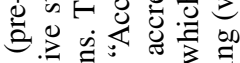

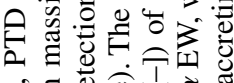
ธ。

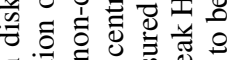
음

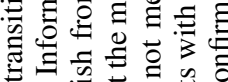
E

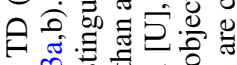

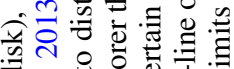

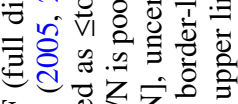

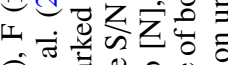
宅宁苋导

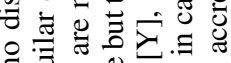

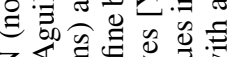

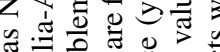

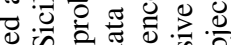

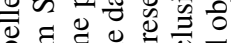

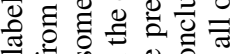

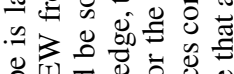

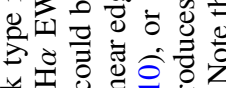

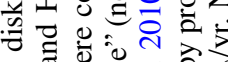
ब न 就影影影 


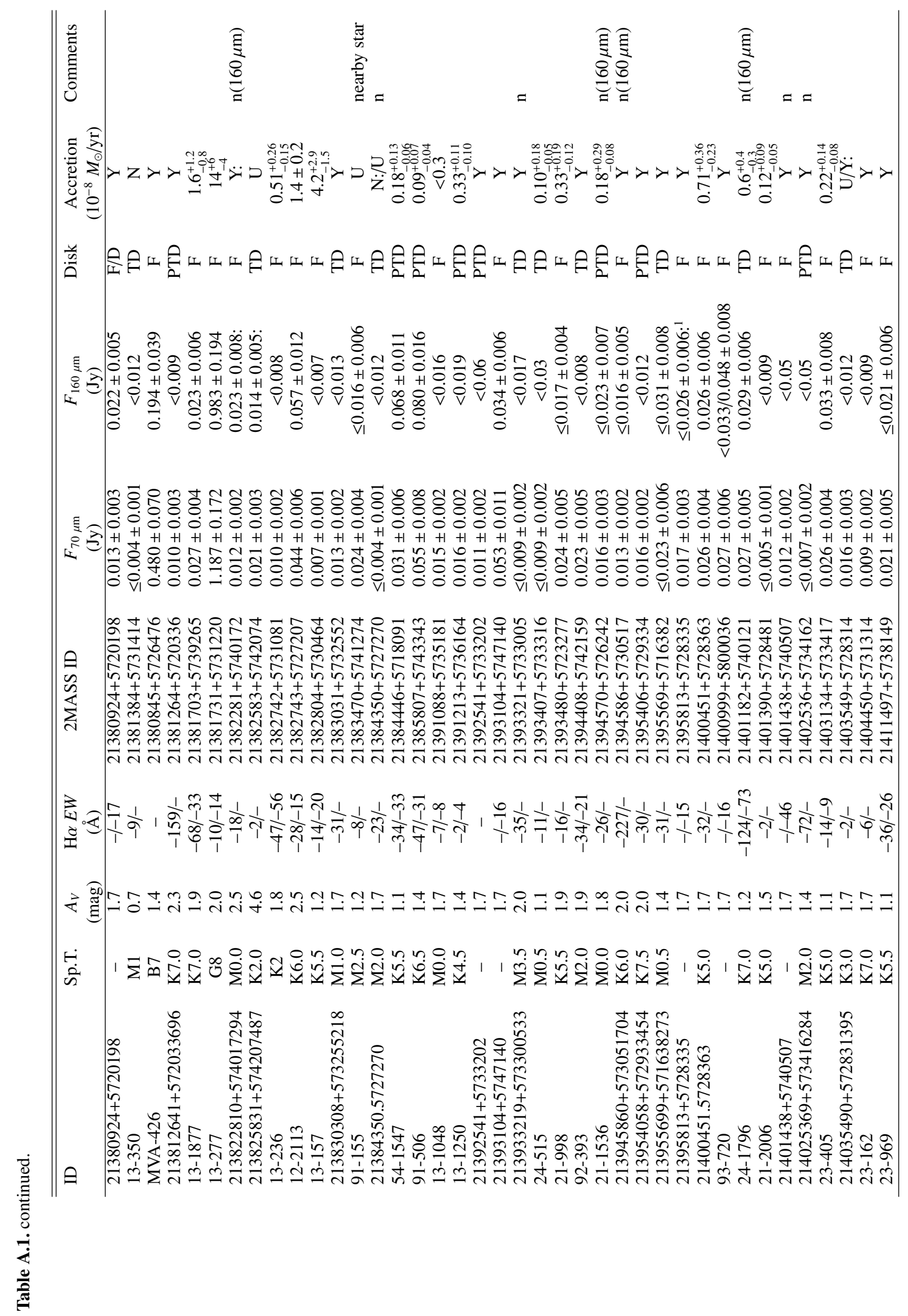


A\&A 573, A19 (2015)

Table A.2. PACS photometry of CepOB2 members identified by Spitzer/X-ray/H $\alpha$ photometry.

\begin{tabular}{|c|c|c|c|c|c|c|}
\hline 2MASS ID & Sp.T. & $F_{70 \mu \mathrm{m}}(\mathrm{Jy})$ & $F_{160 \mu \mathrm{m}}(\mathrm{Jy})$ & Ref. & Disk & Comments \\
\hline $21351021+5731475$ & - & $\leq 0.010 \pm 0.002$ & $<0.027$ & B11 & - & No IRAC data \\
\hline $21351687+5732422$ & late $\mathrm{K} / \mathrm{M}$ & $0.009 \pm 0.002$ & $<0.045$ & B11 & - & \\
\hline $21352723+5731301$ & $\mathrm{~K} / \mathrm{M}$ & $0.016 \pm 0.003$ & $<0.036$ & B11 & - & \\
\hline $21353135+5731279$ & early M & $\leq 0.010 \pm 0.002$ & $<0.015$ & B11 & TD & \\
\hline $21354586+5736401$ & late $\mathrm{K} / \mathrm{M}$ & $\leq 0.009 \pm 0.002$ & $<0.015$ & B11 & $\mathrm{F}$ & \\
\hline $21363802+5726579$ & late $\mathrm{M}$ & $\leq 0.011 \pm 0.003$ & $<0.052$ & MC09 & $\mathrm{F}$ & High extinction \\
\hline $21364964+5722270$ & $\mathrm{~F} / \mathrm{G}$ & $0.033 \pm 0.007$ & $0.039 \pm 0.010$ & G12 & $\mathrm{TD}$ & \\
\hline $21370909+5725485$ & late $\mathrm{K} / \mathrm{M}$ & $0.023 \pm 0.004$ & $0.054 \pm 0.012$ & $\mathrm{~B} 11$ & TD & $\mathrm{n}$ \\
\hline $21371389+5727270$ & late $\mathrm{K}$ & $0.040 \pm 0.006$ & $0.037 \pm 0.009$ & B11 & $\mathrm{F}$ & \\
\hline $21371420+5736177$ & $\mathrm{~K} / \mathrm{M}$ & $0.013 \pm 0.002$ & $<0.048$ & G12 & $\mathrm{F}$ & \\
\hline $21371545+5727170$ & $\mathrm{~K} / \mathrm{M}$ & $0.025 \pm 0.004$ & $0.049 \pm 0.012$ & G12 & F/PTD & $\mathrm{n}$ \\
\hline $21372152+5726123$ & late $\mathrm{K} / \mathrm{M}$ & $0.015 \pm 0.003$ & $<0.047$ & G12 & $\mathrm{F}$ & \\
\hline $21372475+5729089$ & early M & $\leq 0.007 \pm 0.002$ & $<0.022$ & B11 & $\mathrm{F}$ & $\mathrm{n}$ \\
\hline $21373786+5728467$ & mid $\mathrm{K}$ & $0.009 \pm 0.001: 1$ & $<0.019$ & G12 & $\mathrm{N}:{ }^{1}$ & $\mathrm{n}$ \\
\hline $21373885+5732494$ & late $\mathrm{K}$ & $0.008 \pm 0.002:^{1}$ & $<0.021$ & G21 & $\mathrm{N}:{ }^{1}$ & $\mathrm{n}$ \\
\hline $21374292+5736314$ & $\mathrm{G} / \mathrm{F}$ & $0.405 \pm 0.059$ & $0.338 \pm 0.067$ & G12 & TD & \\
\hline $21374612+5734280$ & early/mid M & $\leq 0.005 \pm 0.001$ & $<0.012$ & $\mathrm{~B} 11$ & $\mathrm{~F}$ & \\
\hline $21385760+5729205$ & O6.5 & $0.013 \pm 0.002$ & $<0.09$ & M09 & $\mathrm{N}$ & HD 206267 \\
\hline $21385963+5730080$ & late $\mathrm{K}$ & $0.006 \pm 0.001:$ & $<0.018$ & M09 & $\mathrm{F}$ & \\
\hline $21390321+5730420$ & late $\mathrm{K} / \mathrm{M}$ & $0.004 \pm 0.001:$ & $<0.12$ & B11 & $\mathrm{F}$ & No optical counterpart \\
\hline
\end{tabular}

Notes. All spectral types (except the one from HD 206267) are derived from SED fitting and thus subject to large uncertainties, assuming the extinction will be within the usual cluster values $A_{V}=1-3$ mag. References: M09 (Mercer et al. 2009); MC09 (Morales-Calderón et al. 2009); B11 (Barentsen et al. 2011); G12 (Getman et al. 2012). The disk type is labelled as N (no disk), F (full disk), TD (transition disk), PTD (pretransitional disk), D (depleted disk), - (not enough information). The comments include "m" (marginal detection, marked as $\leq$ to distinguish from non-detection), " $\mathrm{n}$ " (nebular contamination in the region resulting in very high upper limits), "e" (near edge, the data are fine but the S/N is poorer than at the map centre). ${ }^{(1)}$ Given the presence of cloud and the absence of mid-IR excess, the PACS excess is most likely nebular and the objects do not have evidence of disks. 
A. Sicilia-Aguilar et al.: Herschel observations of Cep OB2

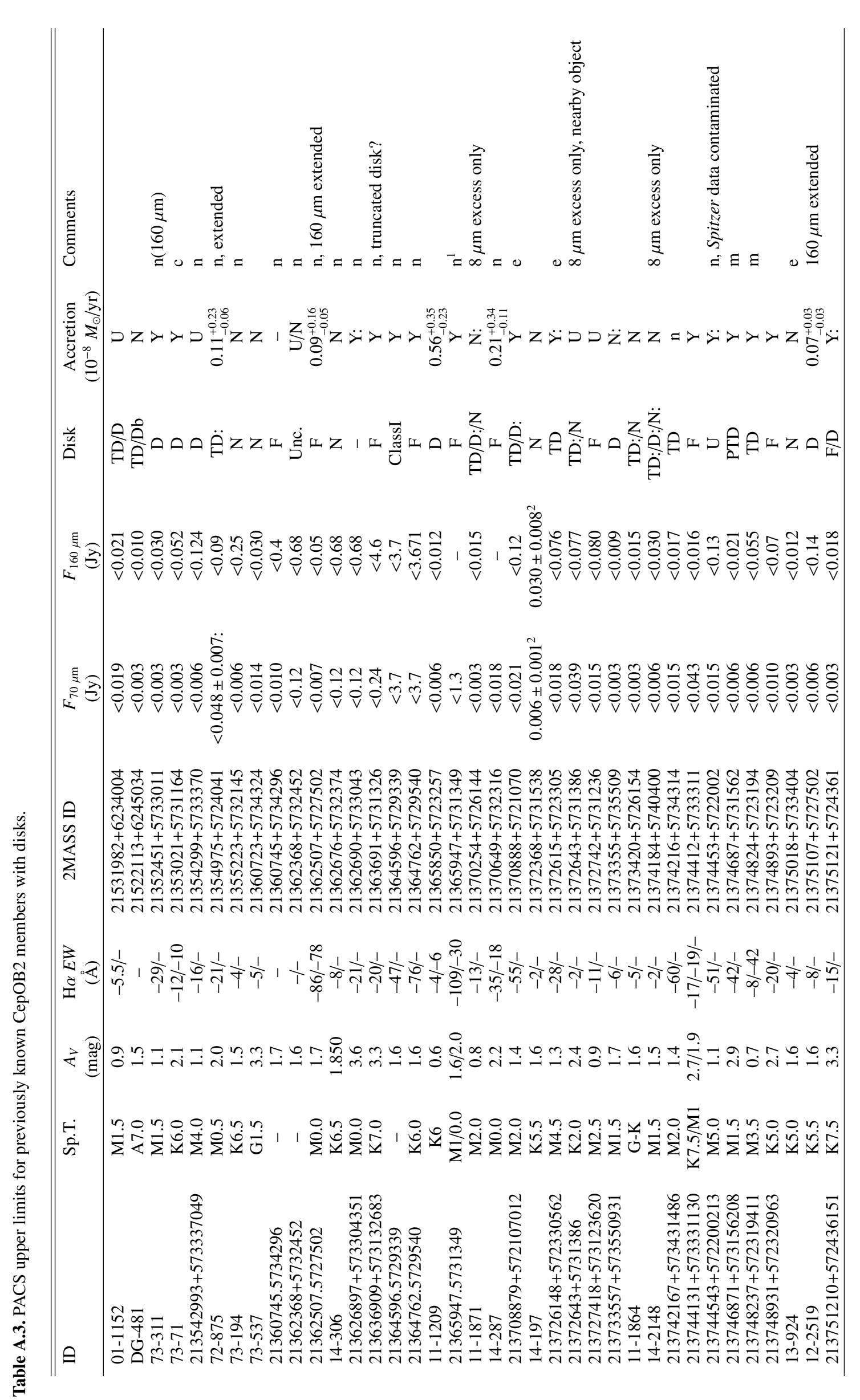

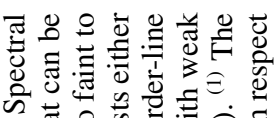

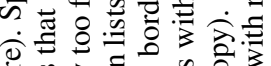

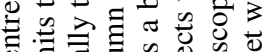

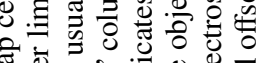
(1)

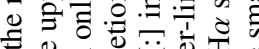

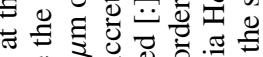
₹ \& $\infty$ <

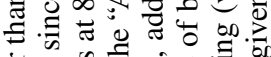

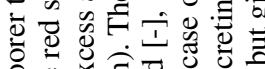
을 을

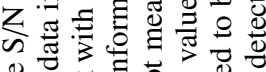

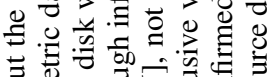
言

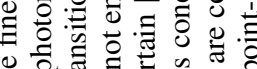

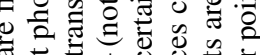

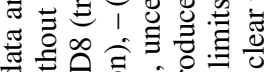

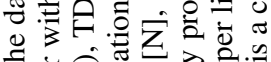

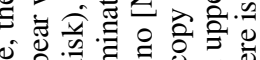

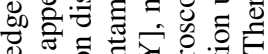

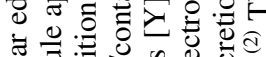

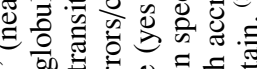
कo

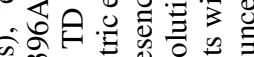

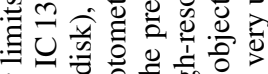

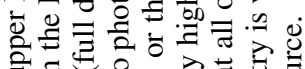

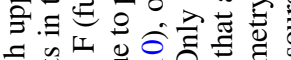

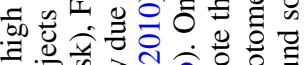

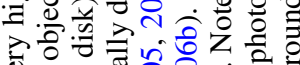

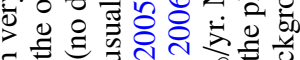

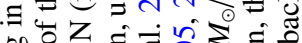

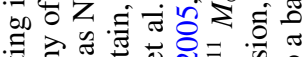

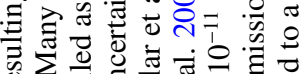

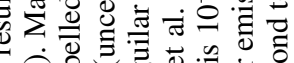
co

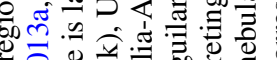

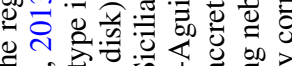
等 告

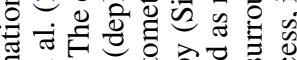
ज。

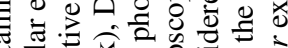

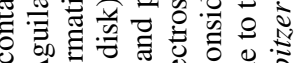

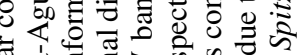
音.

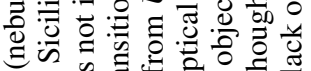
= 흔

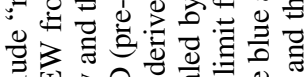

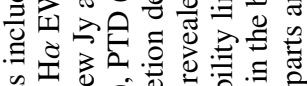

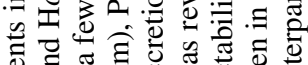

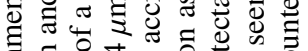

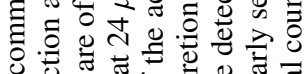

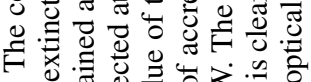

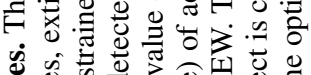

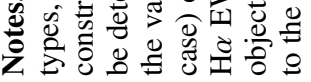




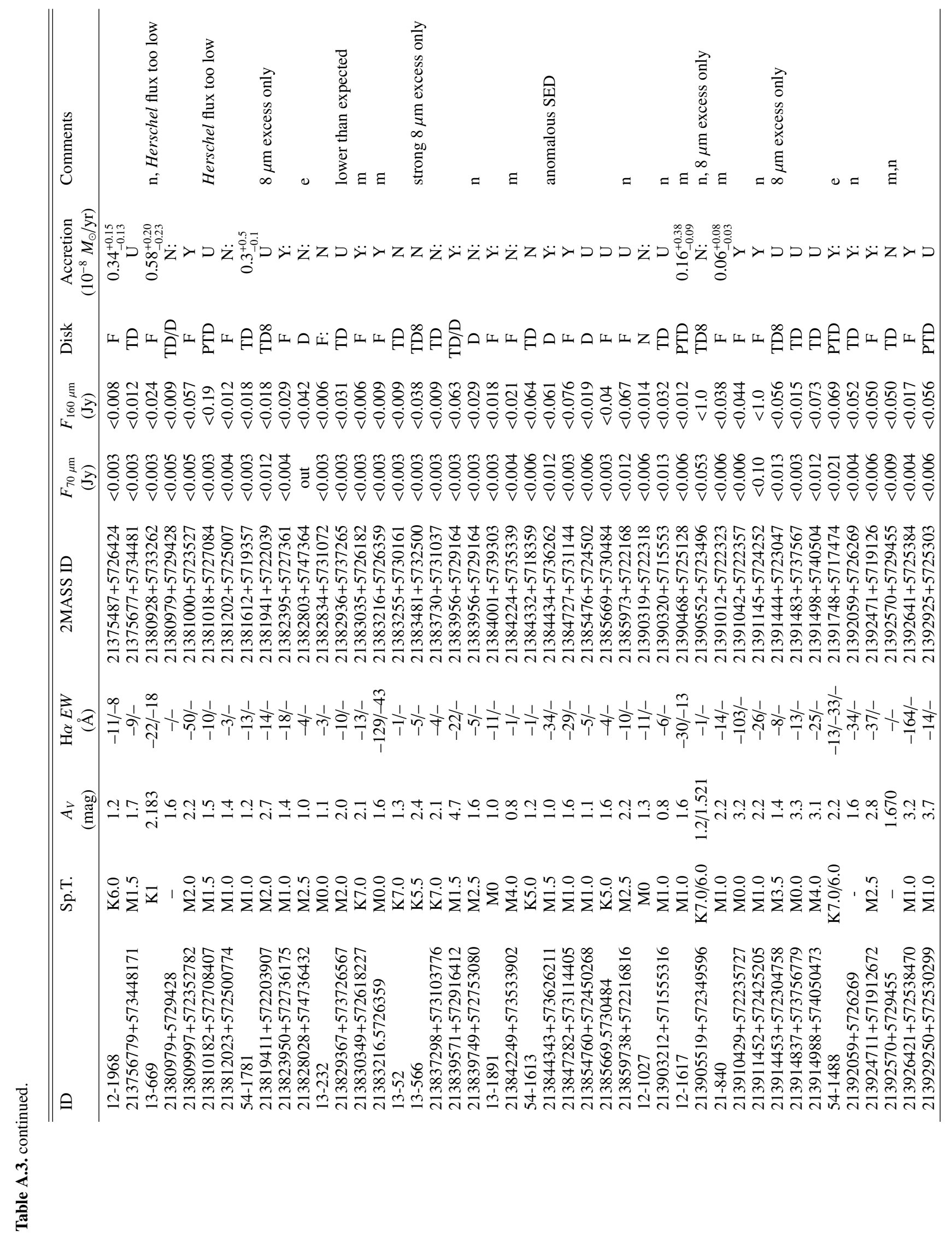




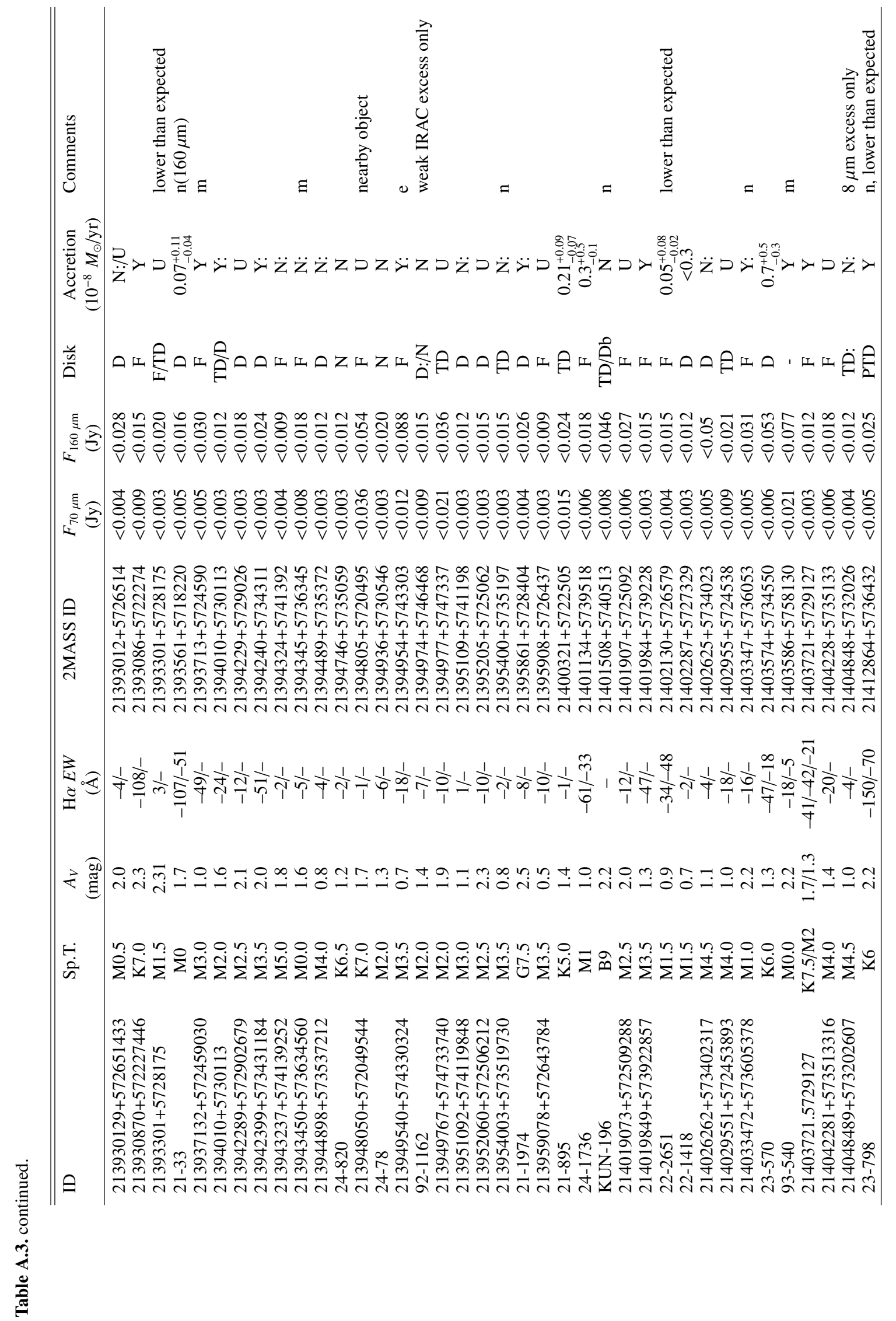


A\&A 573, A19 (2015)

Table A.4. PACS upper limits of CepOB2 members identified by Spitzer/X-ray/H $\alpha$ photometry.

\begin{tabular}{|c|c|c|c|c|c|c|}
\hline 2MASS ID & Sp.T & $F_{70 \mu \mathrm{m}}(\mathrm{Jy})$ & $F_{160 \mu \mathrm{m}}(\mathrm{Jy})$ & Ref. & Disk & Comments \\
\hline $21353289+5731595$ & early $\mathrm{M}$ & $<0.005$ & $<0.078$ & B11 & F/PTD: & \\
\hline $21364033+5725455$ & late $\mathrm{M}$ & $<0.006$ & $<0.067$ & MC09 & $\mathrm{F}$ & high extinction \\
\hline $21364247+5725231$ & $\operatorname{mid} \mathrm{M}$ & $<0.004$ & $<0.030$ & MC09 & F & \\
\hline $21365616+5726395$ & - & $<0.004$ & $<0.032$ & G12 & Unc./N & \\
\hline $21371040+5729034$ & - & $<0.005$ & - & G12 & Unc. & $\mathrm{n}$ \\
\hline $21371474+5728149$ & early $\mathrm{M}$ & $<0.005$ & $<0.017$ & G12 & $\mathrm{TD}$ & \\
\hline $21371689+5729199^{(a)}$ & - & $<0.006$ & - & MC09 & F: & two nearby objects \\
\hline $21371695+5732209$ & - & $<0.004$ & $<0.024$ & G12 & Unc. & \\
\hline $21371700+5726178$ & $\mathrm{~K}$ & $<0.006$ & $<0.066$ & G12 & $\mathrm{F}$ & \\
\hline $21371714+5728473$ & late $\mathrm{K}$ & $<0.005$ & $<0.031$ & B11 & F/PTD: & \\
\hline $21371742+5729272$ & late $\mathrm{K}$ & $<0.007$ & - & MC09 & $\mathrm{F}$ & nearby object \\
\hline $21371996+5728462$ & late $\mathrm{K}$ & $<0.006$ & $<0.031$ & G12 & TD:/N & \\
\hline $21372003+5729344$ & $\mathrm{~K}$ & $<0.005$ & $<0.060$ & G12 & TD/PTD: & \\
\hline $21372009+5731351$ & M & $<0.006$ & $<0.018$ & G12 & $\mathrm{TD}: / \mathrm{N}$ & \\
\hline $21372181+5734122$ & - & $<0.005$ & $<0.059$ & G12 & Unc. & \\
\hline $21373287+5728270$ & early M & $<0.004$ & $<0.011$ & G12 & TD/D: & \\
\hline $21373380+5732166$ & $\mathrm{mid} / \mathrm{late} \mathrm{M}$ & $<0.004$ & $<0.10$ & G12 & $\mathrm{N}:$ & \\
\hline $21374288+5734461$ & M: & $<0.005$ & $<0.012$ & B11 & $\mathrm{F}$ & \\
\hline $21374851+5729587$ & early M & $<0.005$ & $<0.013$ & G12 & TD:/D & \\
\hline $21374944+5733518$ & - & $<0.003$ & $<0.012$ & G12 & $\mathrm{F}$ & \\
\hline $21375256+5725562$ & early/mid M & $<0.005$ & $<0.021$ & G12 & TD & \\
\hline $21375487+5726424$ & K & $<0.004$ & $<0.015$ & G12 & TD & \\
\hline $21383145+5729012$ & late K/early M & $<0.004$ & $<0.025$ & M09 & TD & \\
\hline $21383743+5730206$ & early M & $<0.004$ & $<0.010$ & M09 & $\mathrm{F}$ & \\
\hline $21384370+5731032$ & late K/early M & $<0.003$ & $<0.076$ & M09 & TD:/N & $\mathrm{m}$ \\
\hline $21385093+5728324$ & late $\mathrm{K}$ & $<0.003$ & $<0.088$ & M09 & D & \\
\hline $21385732+5730254$ & early M & $<0.005$ & $<0.059$ & M09 & TD & \\
\hline $21385999+5730532$ & $\mathrm{~K}$ & $<0.005$ & $<0.051$ & M09 & $\mathrm{F}$ & \\
\hline $21390106+5730231$ & $\mathrm{~K} / \mathrm{M}$ & $<0.003$ & $<0.023$ & M09 & TD & \\
\hline $21390624+5728106$ & $\mathrm{~K} / \mathrm{M}$ & $<0.003$ & $<0.021$ & M09 & $\mathrm{F}$ & \\
\hline $21391732+5730085$ & $\mathrm{~K}$ & $<0.004$ & $<0.012$ & M09 & $F / D$ & \\
\hline $21395187+5726583$ & early M & $<0.004$ & $<0.010$ & B11 & $\mathrm{TD} / \mathrm{D}$ & \\
\hline $21395611+5727079$ & late K/early M & $<0.004$ & $<0.011$ & B11 & TD/D: & \\
\hline $21395767+5723433$ & late K/early M & $<0.006$ & $<0.013$ & B11 & TD & \\
\hline $21404073+5732147$ & late K/early M & $<0.004$ & $<0.017$ & B11 & $\mathrm{F}$ & \\
\hline
\end{tabular}

Notes. All spectral types are derived from SED fitting and thus subject to large uncertainties, assuming the extinction will be within the usual cluster values $A_{V}=1-3 \mathrm{mag}$.

References. M09 (Mercer et al. 2009); MC09 (Morales-Calderón et al. 2009); B11 (Barentsen et al. 2011); G12 (Getman et al. 2012). The disk type is labelled as N (no disk), F (full disk), TD (transition disk), PTD (pre-transitional disk), D (depleted disk), - (not enough information). The comments include "m" (marginal detection, marked as $\leq$ to distinguish from non-detection), " $n$ " (nebular contamination in the region resulting in very high upper limits), "e" (near edge, the data are fine but the S/N is poorer than at the map centre). ${ }^{(a)}$ A nearby source $21371737+5729207$ has bright excess, original coordinates correspond to a point between both objects. 

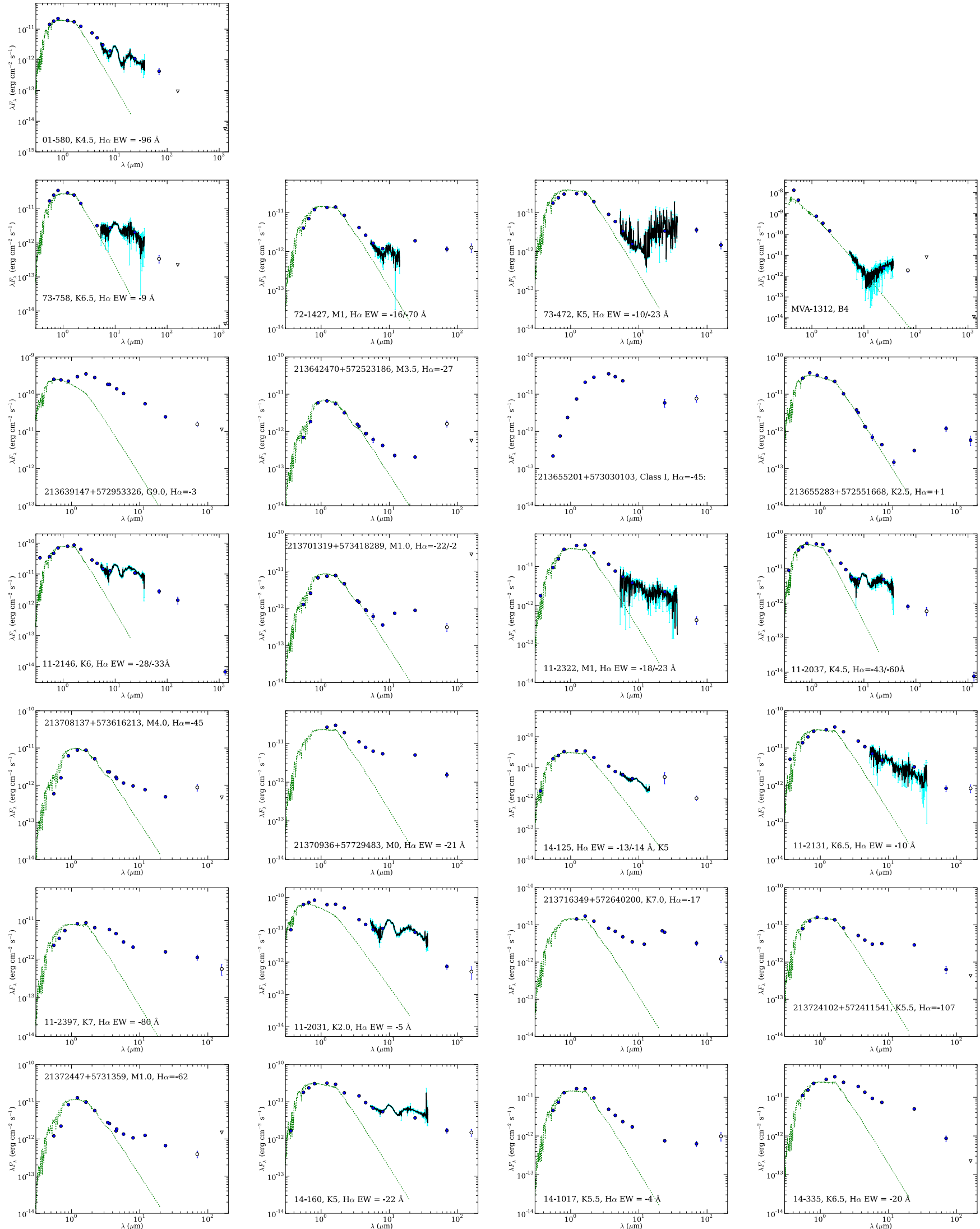

Fig. A.1. SEDs of the objects detected with Herschel, including available optical, Spitzer (IRAC/MIPS photometry and IRS spectra), WISE, and IRAM $1.3 \mathrm{~mm}$ data. The first row contains the only NGC 7160 member detected with Herschel (01-580), the rest belong to Tr 37 . Filled symbols mark detections at different wavelengths. Errorbars are shown in blue for both photometry points (often smaller than the symbols) and the IRS spectra. Upper limits are marked as inverted open triangles. Marginal detections (close to $3 \sigma$ or affected by nebulosity) are marked as open circles. The information about spectral types and $\mathrm{H} \alpha$ emission from the literature is also listed. A photospheric MARCS model is displayed as a dotted line for comparison. 
A\&A 573, A19 (2015)
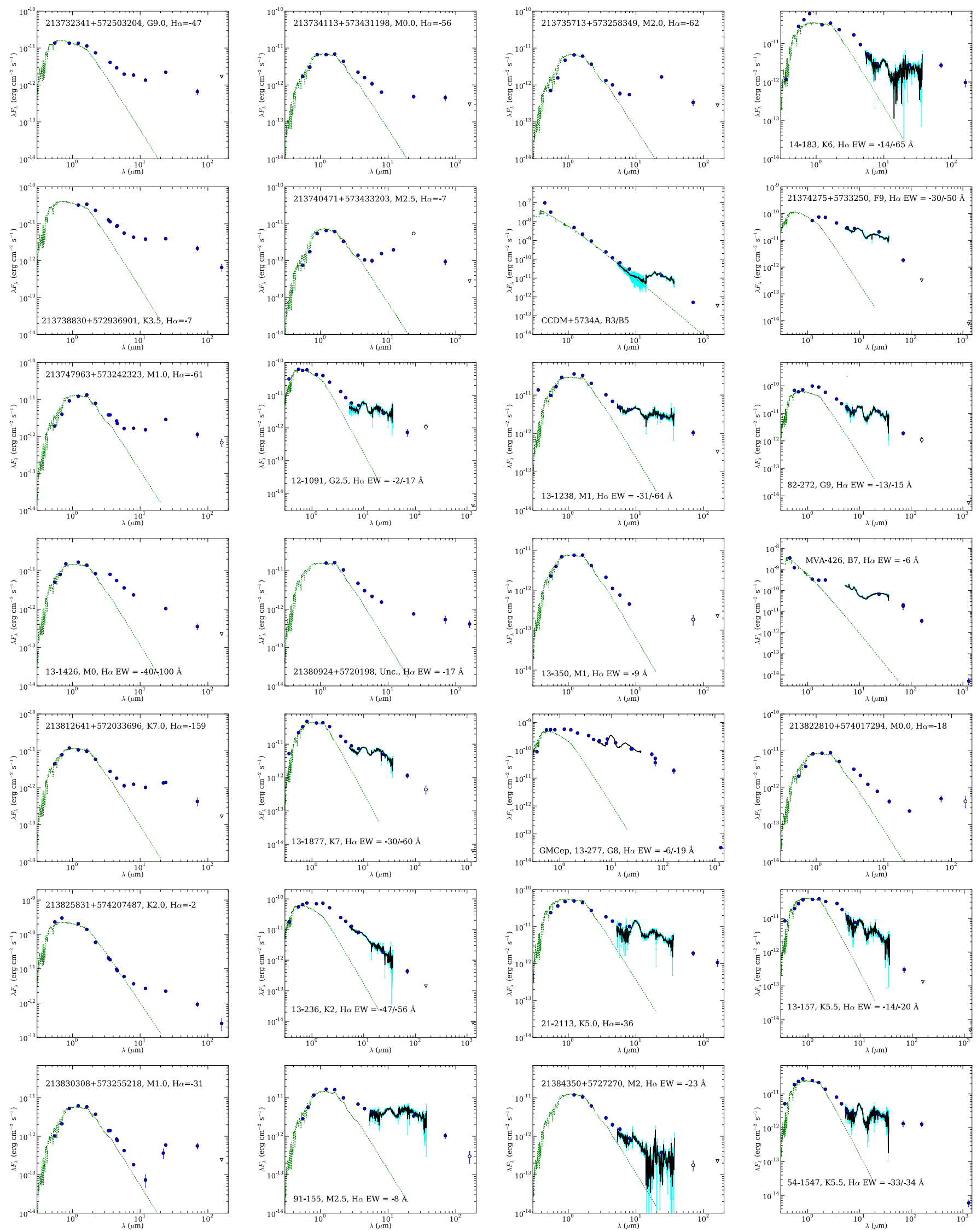

Fig. A.2. SEDs of the objects detected with Herschel (continuation). All objects belong to Tr 37. Symbols as in Fig. A.1. 
A. Sicilia-Aguilar et al.: Herschel observations of Cep OB2
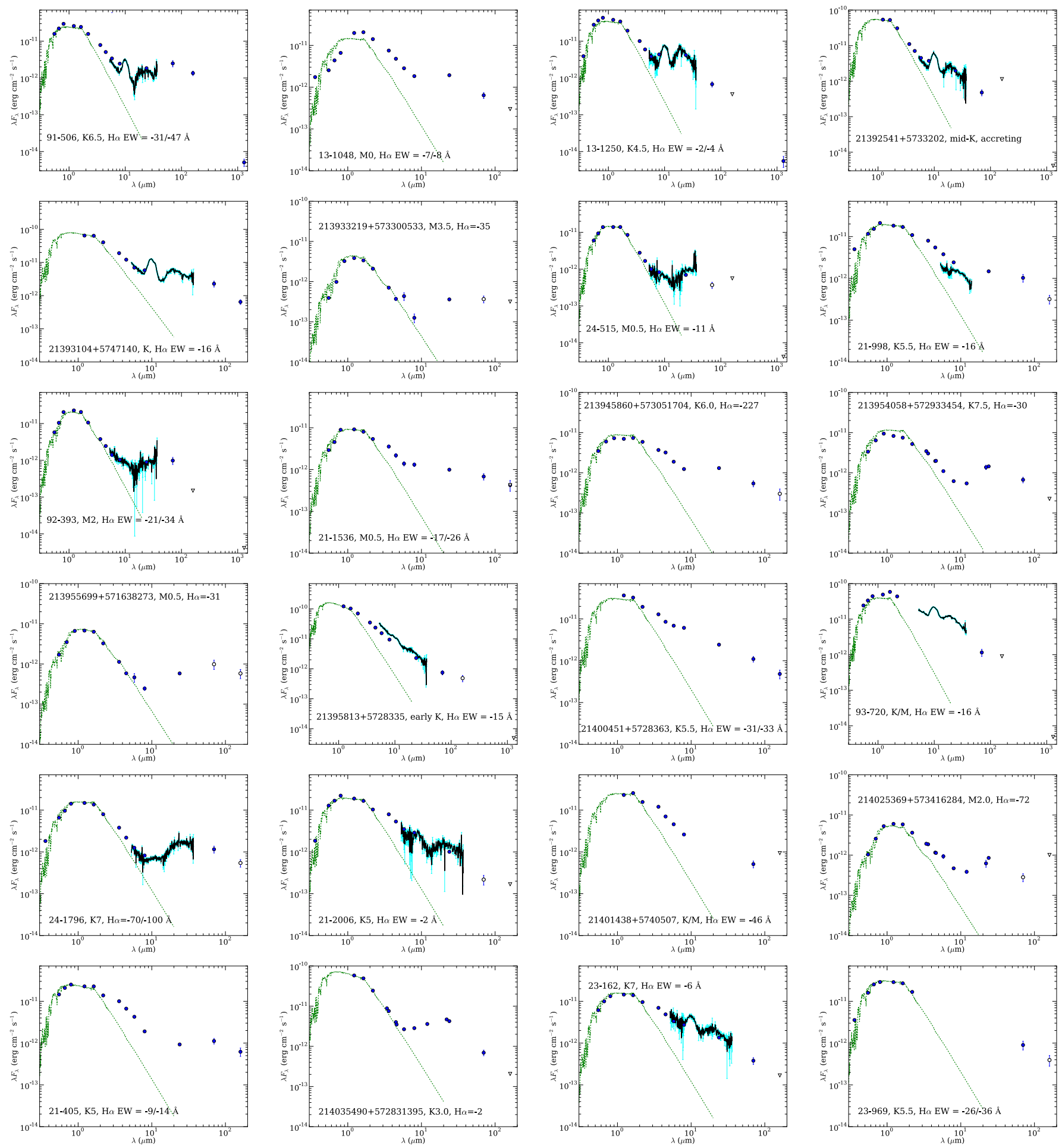

Fig. A.3. SEDs of the objects detected with Herschel (continuation). All objects belong to Tr 37. Symbols as in Fig. A.1. 
A\&A 573, A19 (2015)
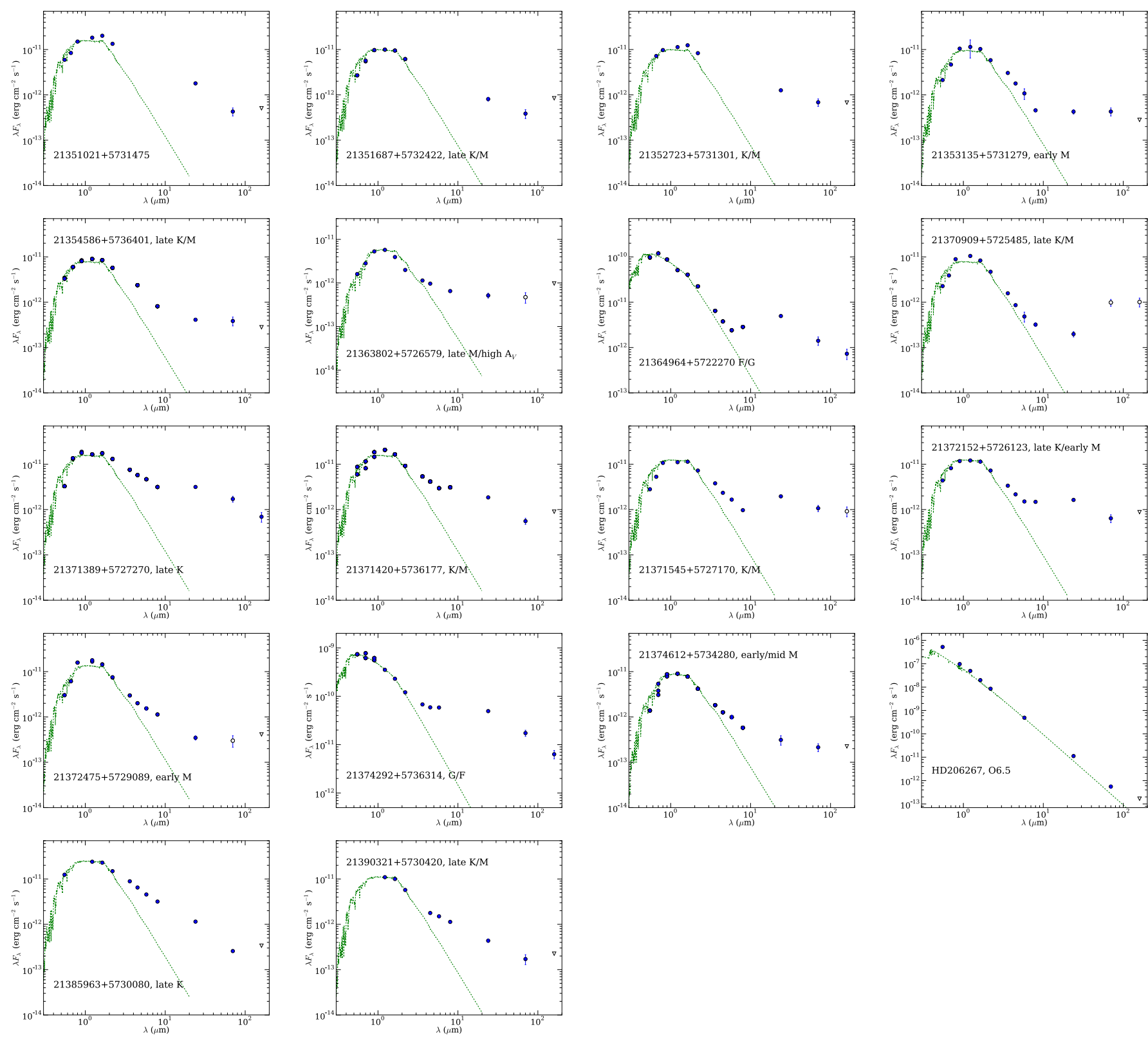

Fig. A.4. SEDs of objects without optical confirmation (see Table A.2) detected with Herschel. Available optical, Spitzer (IRAC/MIPS photometry and IRS spectra), and WISE, data are shown as filled dots. Errorbars are shown (although they are often smaller than the symbols). Upper limits are marked as inverted open triangles. Marginal detections (close to $3 \sigma$ or affected by nebulosity) are marked as open circles. A photospheric MARCS model is displayed for comparison. Approximate spectral types (except for HD 206267 are estimated from the comparison with the MARCS model, assuming the typical cluster extinctions. 

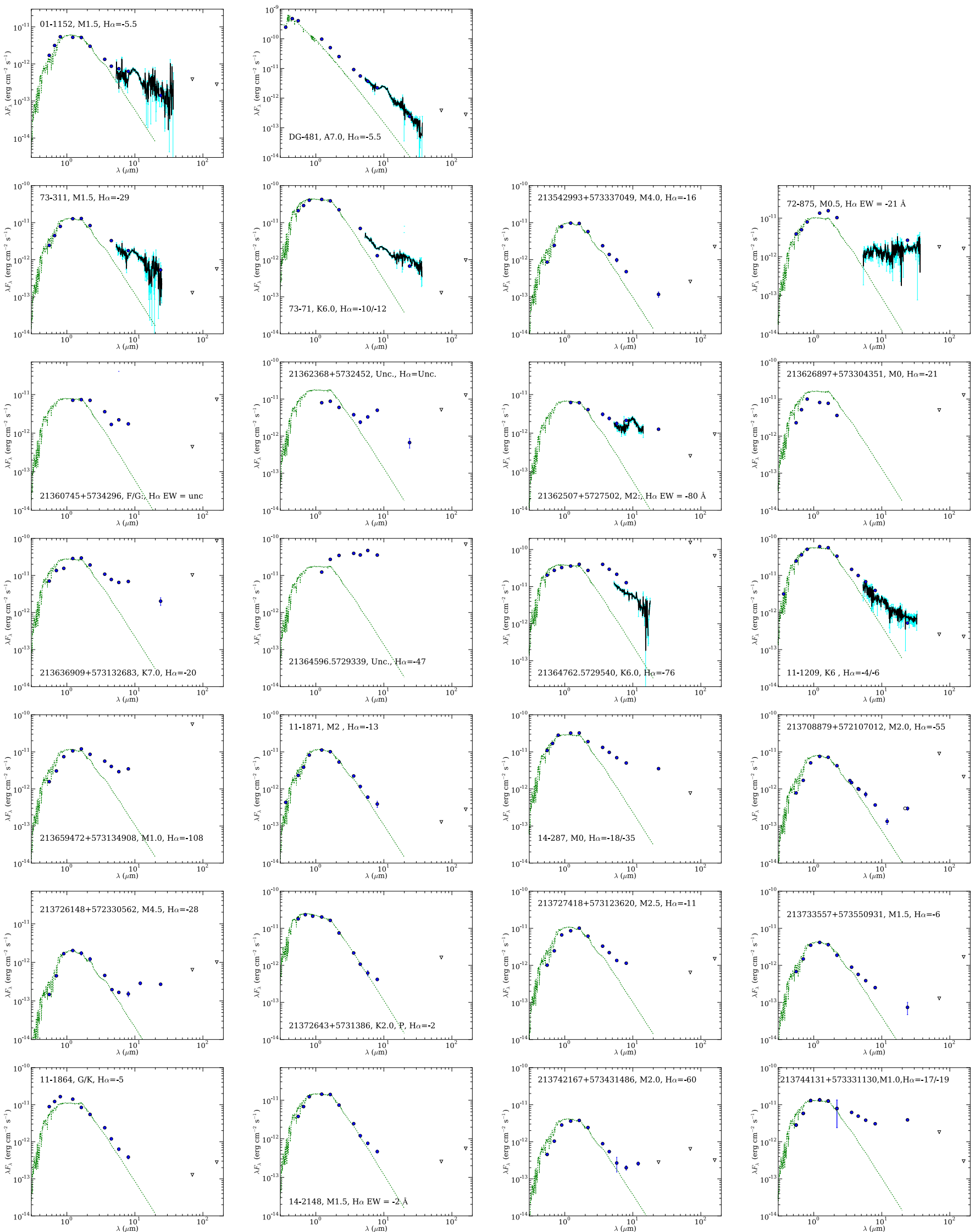

Fig. A.5. SEDs of the objects with upper limits only. The first two objects are in the NGC 7160 cluster, the rest are in Tr 37 . Only objects with confirmed disks (Spitzer excesses) are displayed. Filled symbols mark detections at different wavelengths. Errorbars are shown in blue for both photometry points (often smaller than the symbols) and the IRS spectra. Upper limits are marked as inverted open triangles. Marginal detections (close to $3 \sigma$ or affected by nebulosity) are marked as open circles. The information about spectral types and $\mathrm{H} \alpha$ emission from the literature is also listed. A photospheric MARCS model is displayed for comparison. 
A\&A 573, A19 (2015)
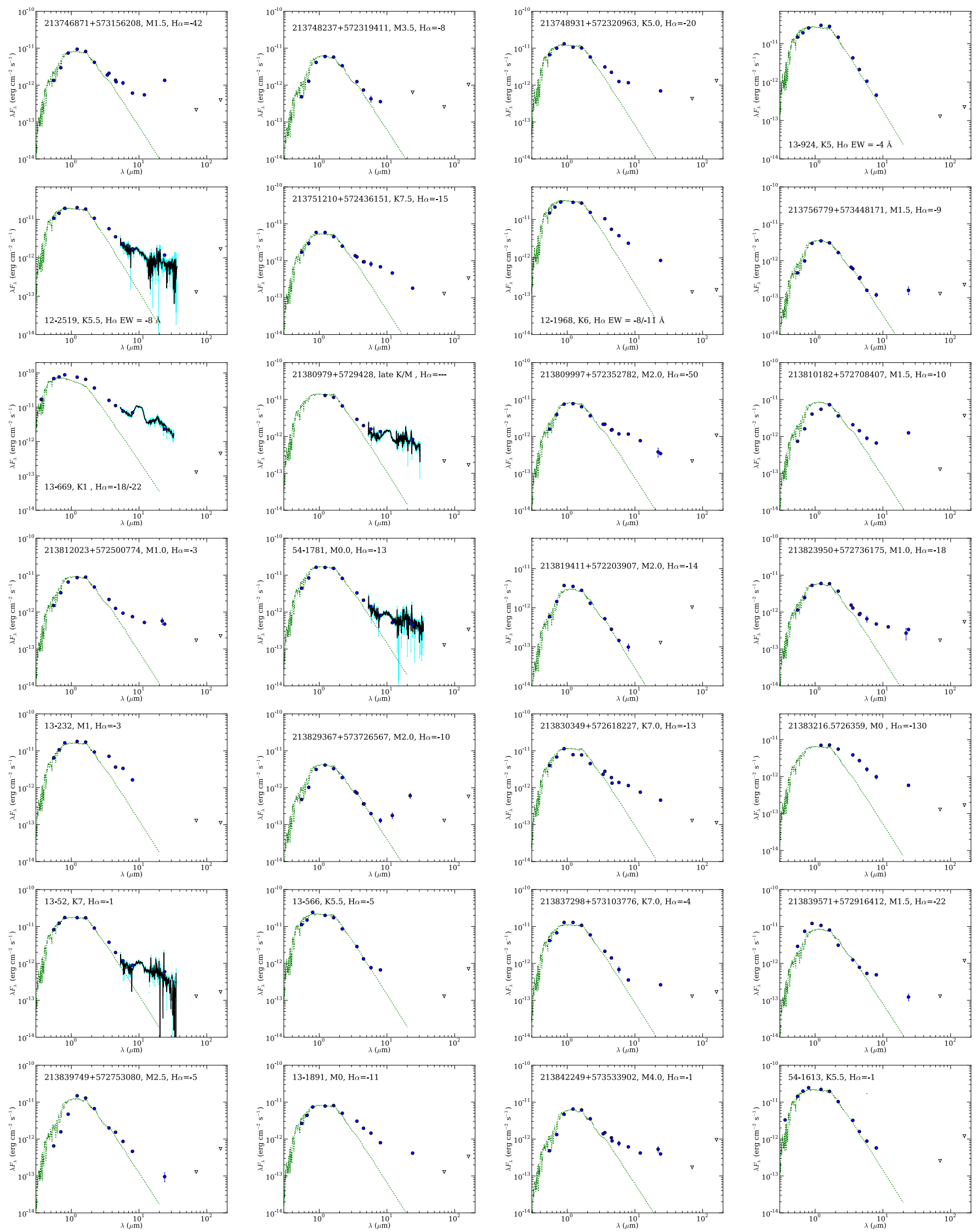

Fig. A.6. SEDs of the objects with upper limits only. Symbols as in Fig. A.5. 

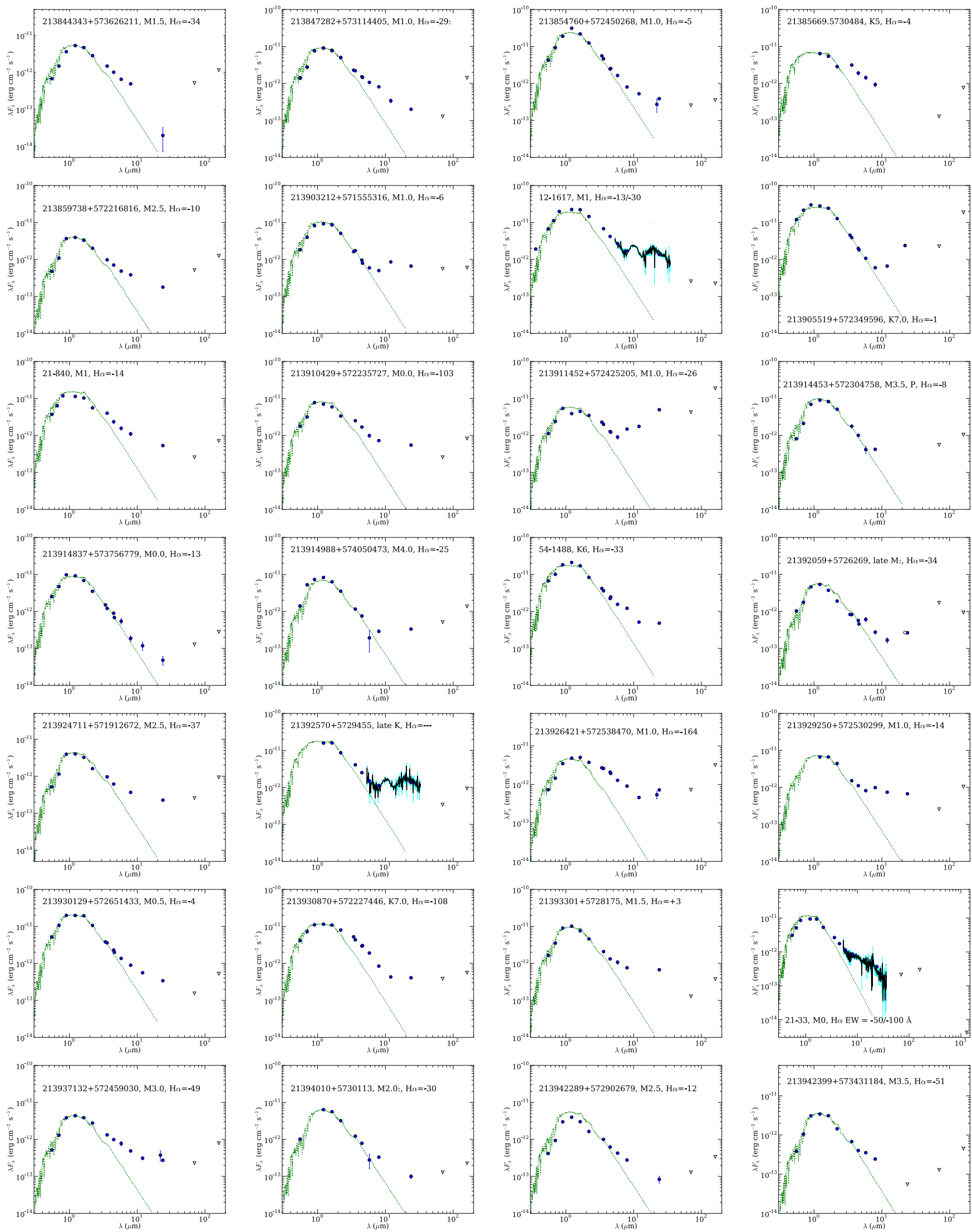

Fig. A.7. SEDs of the objects with upper limits only. Symbols as in Fig. A.5. 
A\&A 573, A19 (2015)


Fig. A.8. SEDs of the objects with upper limits only. Symbols as in Fig. A.5. 
A. Sicilia-Aguilar et al.: Herschel observations of Cep OB2
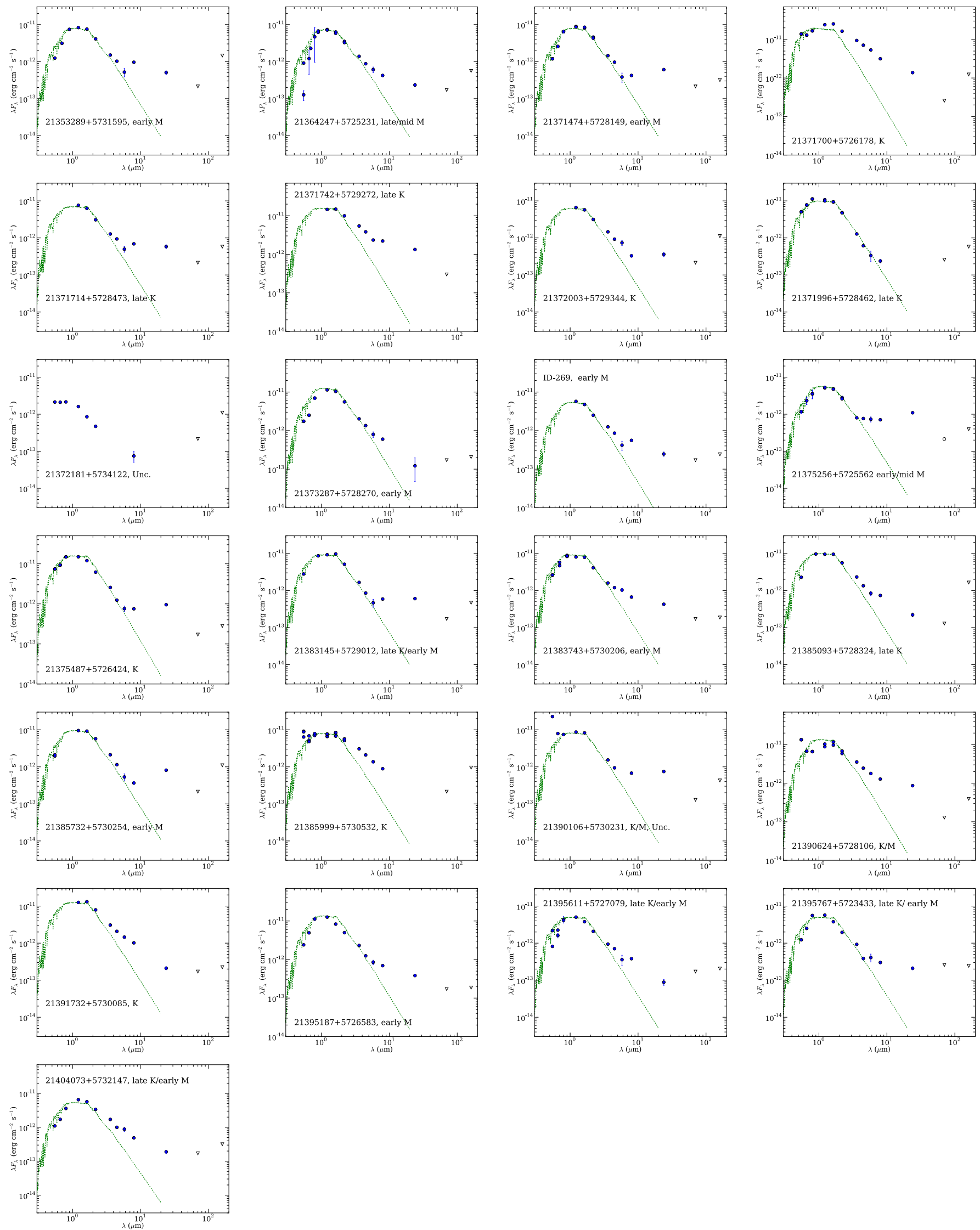

Fig. A.9. SEDs of the objects with Herschel upper limits and without optical spectroscopy confirmation (see Table A.4). Only objects with evidence of a disk at other wavelengths and with significant upper limits (i.e. not contaminated by nearby bright objects/cloud) are listed here. Approximate spectral types (from SED fitting) are listed for the relevant objects. Symbols as in Fig. A.5. 


\section{Appendix B: RADMC models for disks with various disk structures}

The following tables list the properties of the disks models used to interpret the $\alpha$ spectral indices. The disk models do not reproduce any of the CepOB2 members in particular. Instead, we explore the parameter space that samples the behaviours observed in different cluster members. The models are thus an extension of the parameter space explored in Sicilia-Aguilar et al. (2011) for a subsample of representative disks with various SED morphologies. All models were constructed using the 2D radiative-transfer MonteCarlo code RADMC (Dullemond \& Dominik 2004) for a standard star with $T_{\text {eff }}=4275 \mathrm{~K}, R_{*}=1.7 R_{\odot}$, and $M_{*}=1 M_{\odot}$ with a $200 \mathrm{AU}$ disk, seen at a 45 degree inclination. The disks are axisymmetric (2D) but the photon packages are followed in 3D. The RADMC code assumes a disk with well-mixed dust and gas and a standard gas-to-dust ratio of 100. The dust temperatures of all species are the same. The inner rim of the disk is either fixed at the dust destruction temperature $(1500 \mathrm{~K})$ or moved to cooler temperatures to simulate inner holes. A small amount of small dust grains is added at this location to reproduce the observed silicate features. The models can be either vertically iterated to reproduce hydrostatic equilibrium (which washes out any predetermined structure parameters such as vertical pressure scale height $H$ vs. radius or disk thickness, requiring that gas and dust are well-mixed), or to reproduce cases where gas and dust are not well-coupled or there are additional variations in the vertical disk structure. In this latter case, we modify the thickness of the disk $\left(H / R \propto R^{q}\right)$ at the outer disk radius $\left((H / R)_{\text {out }}\right)$ to reproduce thicker/thinner disks, and modify the exponent q to obtain various degrees of flaring or global settling. For the dust content, we assume standard amorphous silicate grains with a grain size distribution between $a_{\min }-a_{\max }$ with a power-law dependency $n(a) \propto a^{-p}$, which can be taken as the standard collisional distribution $(p=-3.5)$ or changed. We also include $25 \%$ of amorphous carbon grains with the same grain distribution as a source of continuum opacity. The grain properties are maintained through the whole disk unless radial variations are included (such as adding small grain populations in the inner disk to reproduce pre-transitional and transitional disks). 


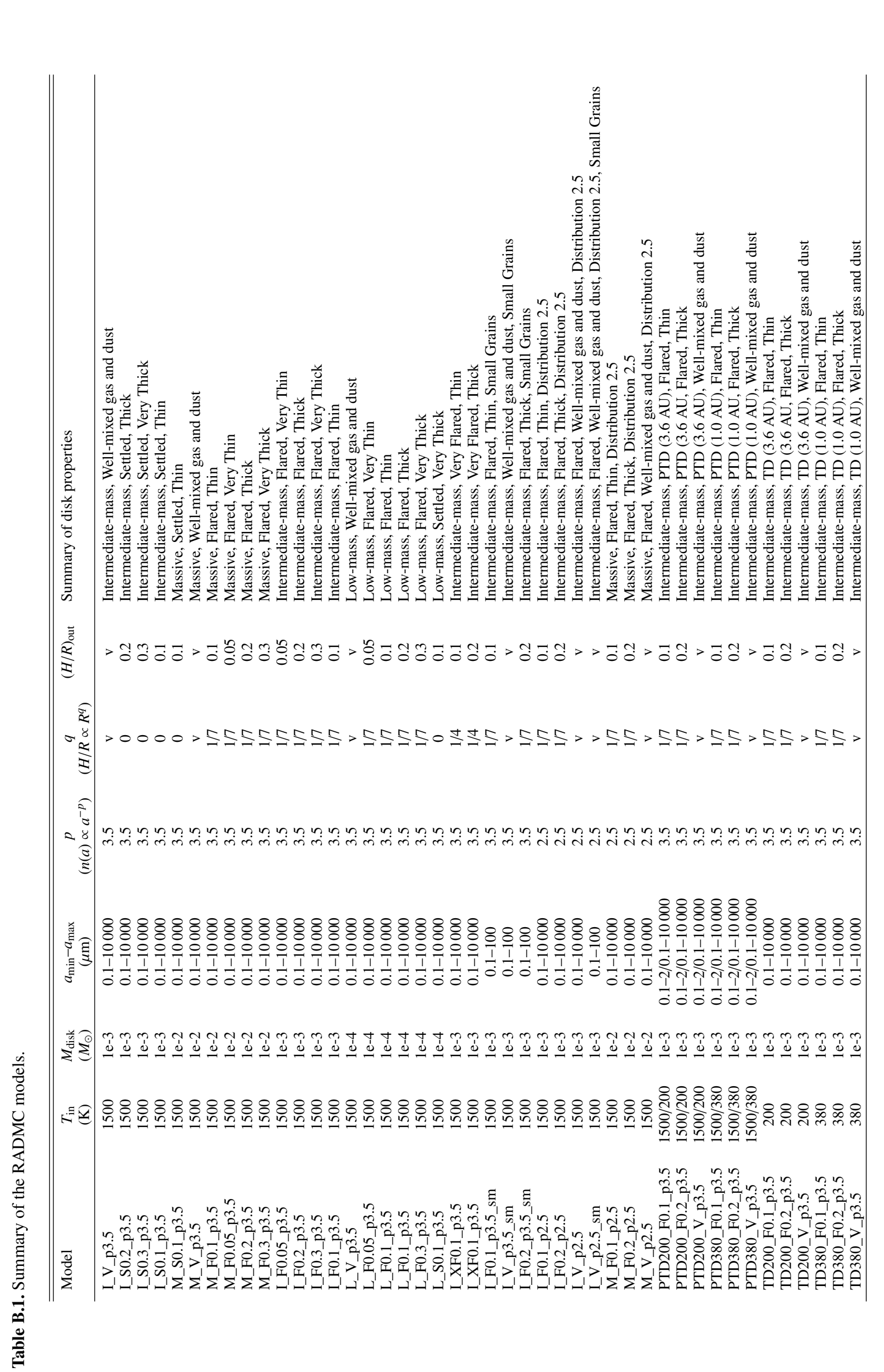

$$
\begin{aligned}
& \text {. }
\end{aligned}
$$

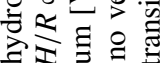

$$
\begin{aligned}
& \text { 品哥言要 }
\end{aligned}
$$

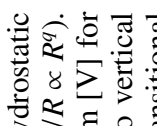$$
\Xi \&:=
$$

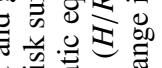$$
\text { 政 }
$$

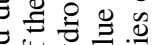$$
\text { 항 }
$$

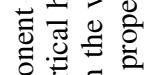$$
\text { 这递哥 }
$$$$
\text { 布 }
$$

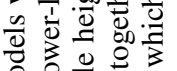

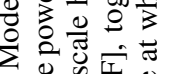

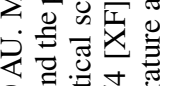

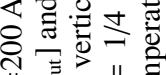

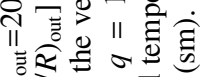
这完焉

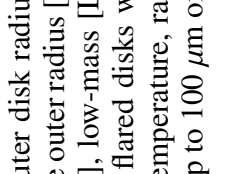

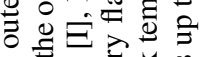

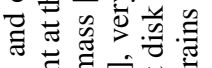

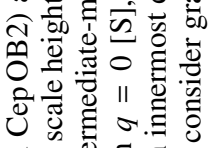

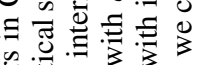

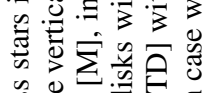

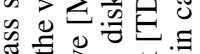

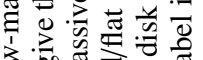

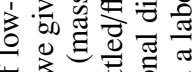

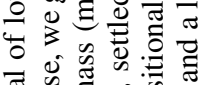

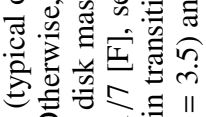

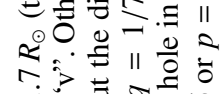

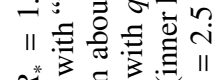

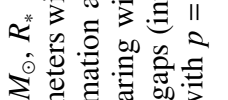

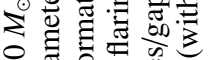

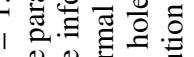

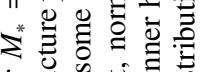

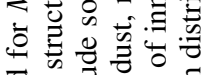

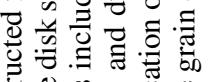

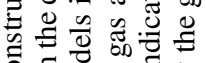

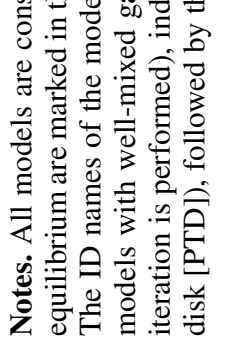


A\&A 573, A19 (2015)

Table B.2. SED $\alpha$ spectral indices for the RADMC models at various wavelengths.

\begin{tabular}{|c|c|c|c|c|c|}
\hline Model & $\alpha(\mathrm{J}-70 \mu \mathrm{m})$ & $\alpha(\mathrm{H}-70 \mu \mathrm{m})$ & $\alpha(8-70 \mu \mathrm{m})$ & $\alpha(24-70 \mu \mathrm{m})$ & $\alpha(70-160 \mu \mathrm{m})$ \\
\hline 1/I_V_p3.5 & -0.84 & -0.90 & -0.33 & -0.65 & -1.77 \\
\hline 2/I_S0.2_p3.5 & -0.91 & -1.01 & -1.07 & -1.30 & -2.07 \\
\hline 3/I_S0.3_p3.5 & -0.80 & -0.92 & -1.12 & -1.39 & -2.17 \\
\hline 4/I_S0.1_p3.5 & -1.07 & -1.17 & -1.04 & -1.20 & -1.89 \\
\hline 5/M_S0.1_p3.5 & -0.86 & -0.95 & -1.10 & -1.35 & -1.28 \\
\hline 6/M_V_p3.5 & -0.64 & -0.69 & -0.03 & -0.14 & -0.80 \\
\hline 7/M_F0.1_p3.5 & -0.85 & -0.93 & -0.62 & -0.70 & -0.80 \\
\hline 8/M_F0.05_p3.5 & -1.04 & -1.12 & -0.75 & -0.80 & -0.68 \\
\hline 9/M_F0.2_p3.5 & -0.66 & -0.73 & -0.59 & -0.71 & -1.06 \\
\hline 10/M_F0.3_p3.5 & -0.53 & -0.61 & -0.62 & -0.76 & -1.25 \\
\hline 11/I_F0.05_p3.5 & -1.14 & -1.23 & -0.90 & -1.03 & -1.69 \\
\hline 12/I_F0.2_p3.5 & -0.87 & -0.95 & -0.89 & -1.16 & -1.99 \\
\hline 13/I_F0.3_p3.5 & -0.79 & -0.88 & -0.96 & -1.27 & -2.10 \\
\hline 14/I_F0.1_p3.5 & -1.01 & -1.09 & -0.85 & -1.02 & -1.82 \\
\hline 15/L_V_p3.5 & -1.17 & -1.26 & -0.91 & -1.58 & -2.48 \\
\hline 16/L_F0.05_p3.5 & -1.34 & -1.44 & -1.24 & -1.64 & -2.67 \\
\hline 17/L_F0.1_p3.5 & -1.25 & -1.36 & -1.27 & -1.75 & -2.71 \\
\hline 18/L_F0.2_p3.5 & -1.16 & -1.27 & -1.36 & -1.96 & -2.76 \\
\hline 19/L_F0.3_p3.5 & -1.11 & -1.22 & -1.45 & -2.10 & -2.80 \\
\hline 20/L_S0.1_p3.5 & -1.29 & -1.39 & -1.39 & -1.82 & -2.83 \\
\hline 21/I_XF0.1_p3.5 & -0.89 & -0.95 & -0.50 & -0.74 & -1.74 \\
\hline 22/I_XF0.1_p3.5 & -0.76 & -0.83 & -0.52 & -0.91 & -1.93 \\
\hline 23/I_F0.1_p3.5_sm & -0.83 & -0.90 & -0.51 & -0.41 & -0.77 \\
\hline 24/I_V_p3.5_sm & -0.61 & -0.65 & 0.10 & 0.27 & -0.85 \\
\hline 25/I_F0.2_p3.5_sm & -0.63 & -0.70 & -0.47 & -0.46 & -1.11 \\
\hline 26/I_F0.1_p2.5 & -1.08 & -1.17 & -0.99 & -1.29 & -2.75 \\
\hline 27/I_F0.2_p2.5 & -0.97 & -1.06 & -1.08 & -1.52 & -2.96 \\
\hline 28/I_V_p2.5 & -1.01 & -1.09 & -0.65 & -1.15 & -2.85 \\
\hline 44/I_V_p2.5_sm & -0.89 & -0.96 & -0.43 & -0.63 & -0.77 \\
\hline 29/M_F0.1_p2.5 & -0.91 & -0.99 & -0.73 & -0.92 & -1.95 \\
\hline 30/M_F0.2_p2.5 & -0.76 & -0.84 & -0.77 & -1.05 & -2.28 \\
\hline 31/M_V_p2.5 & -0.80 & -0.86 & -0.32 & -0.65 & -2.15 \\
\hline 32/PTD200_F0.1_p3.5 & -0.91 & -0.98 & -0.54 & -0.73 & -2.13 \\
\hline 33/PTD200_F0.2_p3.5 & -0.68 & -0.73 & -0.28 & -1.28 & -2.49 \\
\hline 34/PTD200_V_p3.5 & -0.84 & -0.90 & -0.21 & -0.88 & -1.96 \\
\hline 35/PTD380_F0.1_p3.5 & -1.01 & -1.08 & -0.71 & -1.09 & -1.89 \\
\hline 36/PTD380_F0.2_p3.5 & -0.80 & -0.86 & -0.82 & -1.39 & -2.18 \\
\hline 37/PTD380_V_p3.5 & -0.89 & -0.95 & -0.40 & -0.59 & -1.71 \\
\hline 38/TD200_F0.1_p3.5 & -0.84 & -0.90 & -0.17 & -1.53 & -2.08 \\
\hline 39/TD200_F0.2_p3.5 & -0.70 & -0.75 & -0.15 & -1.63 & -2.15 \\
\hline 40/TD200_V_p3.5 & -0.88 & -0.94 & -0.19 & -1.43 & -1.76 \\
\hline 41/TD380_F0.1_p3.5 & -0.93 & -0.99 & -0.88 & -1.36 & -1.96 \\
\hline 42/TD380_F0.2_p3.5 & -0.77 & -0.82 & -0.91 & -1.47 & -2.13 \\
\hline 43/TD380_V_p3.5 & -0.89 & -0.95 & -0.62 & -0.81 & -1.60 \\
\hline
\end{tabular}

Notes. SED spectral indices $(\alpha)$ for the disk models in Table B.1. 


\section{Appendix C: Notes on individual objects}

Here we present a comparison of the fluxes predicted by our previous, Spitzer-based disk models (Sicilia-Aguilar et al. 2011; SA13) and the actual Herschel observations. Herschel/PACS fluxes and upper limits are highly consistent with the Spitzer (MIPS/IRS) data. The measured far-IR fluxes are in good agreement with our model predictions based on optical, Spitzer, and millimetre data, although some cases present higher or lower fluxes than expected. Extended emission at $160 \mu \mathrm{m}$ likely affects the fluxes of objects like 72-1427 (Fig. A.1), 21374763+573242323, 12-1091 (Fig. A.2), and probably others like 14-1017 (Fig. A.1), $213945860+573051704$, and $21400451+5728363$ (Fig. A.3), even though all of them are clearly detected at this wavelength. The sharp turn-down/low mid-IR fluxes in objects like 13-1250, 21392541+5733202, 21395813+5728335, and 21-2006 (Fig. A.3) are also confirmed by Herschel/PACS. In particular, 13-1250 shows a remarkably steep SED in the far-IR, a strong silicate feature and low mid-IR fluxes, suggestive of a disk gap. A low far-IR flux could indicate a low dust mass and/or strong flattening of the disk beyond what we had assumed in Spitzer-based models. The star $21374275+5733250$ (Fig. A.2) also shows relatively lower-thanexpected fluxes in the far-IR, although since it is surrounded by nebulosity very close to the bright binary CCDM J2137+5734, its $24 \mu \mathrm{m}$ flux could be contaminated.

Figure C.1 presents the SEDs including the Herschel data together with our previous disk models derived from optical, Spitzer, and millimetre observations alone. Individual disk modelling is beyond the scope of this paper. Further millimetre-wave observations are essential to study the global evolution of these objects in more detail. The 70 and $160 \mu \mathrm{m}$ model flux predictions for objects such as 13-236, 13-52, 24-1796 are in excellent agreement with the observations. In cases such as 213930129+572651433, the upper limits favour an interpretation of the disk in terms of a relatively low-mass/low-flaring, and not simply as a massive but very settled disk. There is some disagreement (around 30\%) between predicted and observed fluxes (or upper limits) in 92-393, 11-1209, $213929250+572530299$ (model predictions slightly higher than observed), and $213735713+573258349$ (model prediction sligthly lower than observed). This confirms that, although subject to strong uncertainties in the disk mass and dust grain properties, the mid-IR based SED classification offers a powerful prediction of the global evolutionary status of most protoplanetary disks.
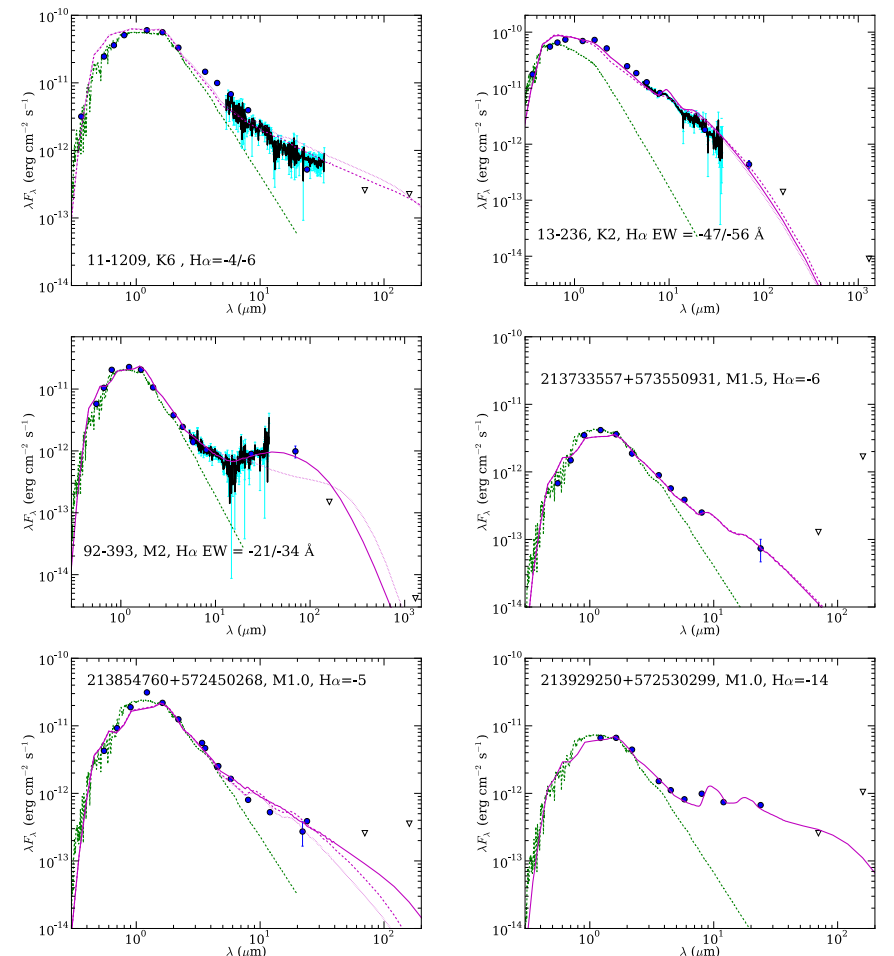
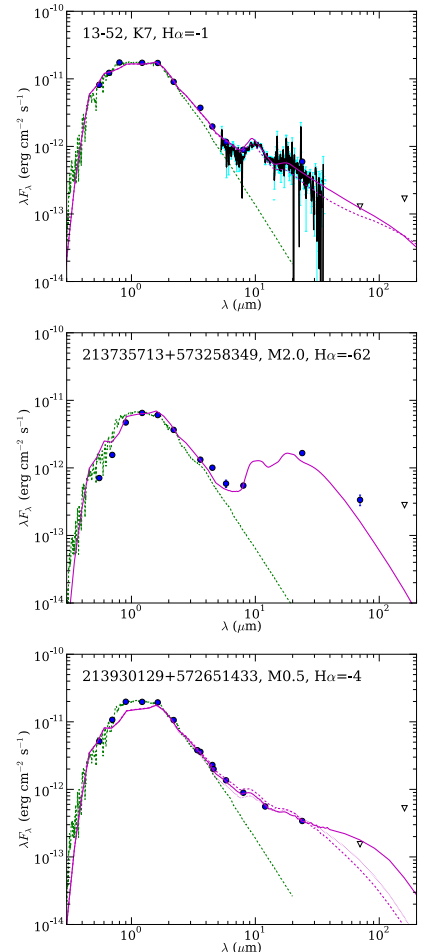
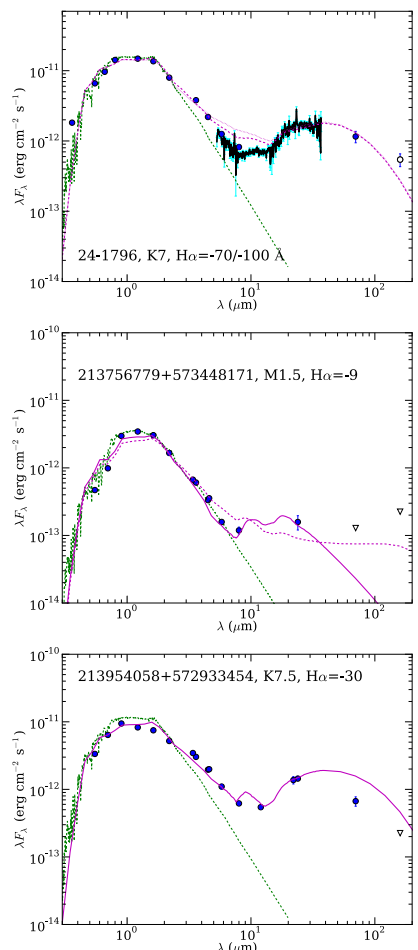

Fig. C.1. Comparison of some of the Spitzer-based SED models (pink lines; Sicilia-Aguilar et al. 2011, 2013a,b) and predicted 70/160 $\mu \mathrm{m}$ fluxes with the actual Herschel/PACS observations. Filled symbols mark detections at different wavelengths. Errorbars are shown in blue for both photometry points (often smaller than the symbols) and the IRS spectra. Upper limits are marked as inverted open triangles. Marginal detections (close to $3 \sigma$ or affected by nebulosity) are marked as open circles. A photospheric MARCS model is displayed as a dotted line for comparison. 